The quality of this microform is heavily dependent upon the quality of the original thesis submitted for microfilming. Every effort has been made to ensure the highest quality of reproduction possible.

If pages are missing, contact the university which granted the degree.

Some pages may have indistinct print especially if the original pages were typed with a poor typewriter ribbon or if the university sent us an inferior photocopy.

Reproduction in full or in part of this microform is governed by the Canadian Copyright Act, R.S.C. 1970, c. C-30, and subsequent amendments.
La qualité de cette microforme dépend grandement de la qualité de la thèse soumise au microfilmage. Nous avons tout fait pour assurer une qualité supérieure de reproduction.

S'il manque des pages, veuillez communiquer avec l'université qui a conféré le grade.

La qualité d'impression de certaines pages peut laisser à désirer, surtout si les pages originales ont été dactylographiées à l'aide d'un ruban usé ou si l'universilié nous a fait parvenir une photocopie de qualité inférieure.

La reproduction, même partielle, de cette microforme est soumise à la Loi canadienne sur le droit d'auteur, SRC 1970, c. C-30, et ses amendements subséquents. 


\title{
Auditory Distortion Measures for Speech Coder Evaluation
}

\author{
by \\ Aloknath De \\ A thesis submitted to the Faculty of the Graduate \\ Studies and Research in partial fulfillment of \\ the requirements for the degree of \\ Doctor of Philosophy \\ Department of Electrical Engineering \\ McGill University \\ Montréal, Canada \\ October, 1993 \\ (C)Aloknath De, 1993
}


The author has granted an irrevocable non-exclusive licence allowing the National Library of Canada to reproduce, loan, distribute or sell copies of his/her thesis by any means and in any form or format, making this thesis available to interested persons.

The author retains ownership of the copyright in his/her thesis. Neither the thesis nor substantial extracts from it may be printed or otherwise reproduced without his/her permission.
L'auteur a accordé une licence irrévocable et non exclusive permettant à la Bibliothèque. nationale du Canada de reproduire, prêter, distribuer ou vendre des copies de sa thèse de quelque manière et sous quelque forme que ce soit pour mettre des exemplaires de cette thèse à la disposition des personnes intéressées.

L'auteur conserve la propriété du droit d'auteur qui protège sa thèse. Ni la thèse ni des extraits substantiels de celle-ci ne doivent être imprimés ou autremeni reproduits sans son autorisation. 
To my grandmother 


\begin{abstract}
One of the important research problems in the area of specch coding is to determine the sound quality of coded speech signals. This quality can best be evaluated by a subjective assessment which is often difficult to administer and time-consuming. An objective measure which is consistent with subjective assessment could play a vital role in the evaluation as well as in the design of a low bit-rate speech coder. In this dissertation, we introduce two distortion measures for specch coder evaluation. Since the perceptual abilities of a human being determine the precision with which speech data must be processed, we consider the details of cochlear (inner ear) and other auditory processing. Using Lyon's auditory model, the time-domain speech signal is mapped onto a perceptual-domain (PD). Any speech utterance is communicated to the brain through a series of all-or-none electrical spikes (firings) and the $\mathrm{PD}$ representation provides information pertaining to the probability-of-firings in the neural channels. Our first measure, namely the cochlear discrimination information (CDI), evaluates the cross-entropy of the neural firings for the coded speech with respect to those for the original one. With this measure, we also compute the ratedistortion function determining the lowest bit-rate required for a specified amount of distortion. In the second measure, namely the cochlear hidden Markovian (CHM) measure, we attempt to capture the high-level processing in the brain with simple hidden Markov models (HMMs). We characterize the firing events by HMMs where the order of occurrence of PD observations and correlations among adjacent observations are modeled suitably. For computing the coder distortion, the PD observations of the coded speech are matched against the HMMs derived from the PD observations of the original speech. Experimental results show that these measures conform to subjective evaluation results in majority of the cases. Finally, the introduced measures are also applied in speech coder analysis, e.g., in the pitch frequency determination and the evaluation of noise weighting schemes.
\end{abstract}




\section{Sommaire}

L'un des problèmes de recherche importants dans le domaine du codage de la parole est de déterminer la qualité de son des signaux de parole codés. Cette qualité est évaluée à son meillcur par un jugement subjectif, ce qui est souvent difficile à organiser et assez long. Une mesure objective consistente avec l'évaluation subjective pourrait jouer un rôle vital dans la conception de codeurs de parole à bas taux de bits ainsi que dans le jugement quailitatif de la parole. Nous introduisons dans cette dissertation deux mesures de distortion pour l'évaluation de performance de codeurs de parole. Etant donné que la précision avec laquelle les données de parole devraient être traitées est déterminée par les capacités perceptuelles de l'être humain, nous considérons les détails du traitement de signaux par la cochlea (intérieur de l'oreille), ainsi que d'autres traitements par le système autitif. En utilisant le modèle auditif de Lyon, le parole dans le domaine temporel est transformée dans le domaine perceptuel (PD). Chaque phrase parlée est communiquée au cerveau à travers une série d'impulsions électriques sur une base de tout ou rien, et la représentation PD offre des informations pertinent à la probabilité d'envoi des impulsions dans les canaux neuronaux. Notre première mesure, plus exactement la discrimination de l'information par la cochlea (CDI), évalue l'entropie croisée des impulsions envoyées pour la parole codée avec ceux de la parole originale. Avec cette mesure, nous calculons aussi une fonction taux-distortion pour déterminer le plus bas taux de bits requis pour un niveau de distortion donné. Dans la seconde mesure de distortion, la Markovienne cachée de la cochlea (CHM), nous essayons de capturer le traitement de haut nivcau dans le cerveau à travers de simples modèles de Markov cachés (HMM). Nous caractérisons les événements d'envoi d'impulsions par des HMM où l'ordre de lieu d'observations PD et la correlation entre observations adjacentes sont proprement représentés. Pour calculer la distortion du codeur, les observations PD de la parole codée sont comparées aux HMMs dérivés des observations PD de la parole d'origine. Les résultats expérimenta:x démontrent que ces mesures sont conforme à l'évaluation subjective dans la majorité des cas. Finalement, les mesures introduites sont appliqués à l'analyse dans le codage de la parole, par exemple, pour la détermination de la fréquence fondamentale et l'évaluation de modèles de pondération bruités. 


\section{Acknowledgements}

I express sincere gratitude to my supervisor Prof. P. Kabal for his technical guidance as well as financial assistance throughout this work. His magnetic personality, logical thinking and pleasant bchavior have helped me overcome many difficult times in my doctoral study. My debt to him is simply immeasurable.

I am thankful to Prof. H. Leib who has contributed to my teaching and research abilities in various ways. I would also like to thank Prof. G. Zames for his healthy advices as one of my $\mathrm{Ph} . \mathrm{D}$. committee members. I am grateful to all the professors and staff members who have assisted me in making my professional career sound.

I owe to all my friends who have helped cherish my four years of stay in Montréal. I extend my heartfelt thanks to Mr. S. Valace and Dr. A. K. Khandani with whom I have had many stimulating discussions. I also thank Mr. N. Maroun for the French translation of the thesis abstract. I have enjoyed the environment of McGill university as well as INRS-Télécommunications. The facilities provided by them have contributed greatly to the accomplishment of this work.

I acknowledge the Canadian Institute for Telecommunicalions Research for sponsoring this project. I am thankful to Dr. M. Slancy of $\Lambda$ pple Computers Inc. for providing me the MacEar program. I would thank the CCITT Specch Quality Expert Group, some members of which gave me an opportunity to introduce this work to them at Orlando, USA. I would also like to thank the Canadian $\Lambda$ coustical Association for recognizing this research work with the ' 1993 Alexander Graham Bell Prize'.

My thanks are due to my dear parents whose loving care and proper ad vices have brought me to the point where I am today. I also express my thanks to my brother Arup and sister Ipsita for their affection. A special thank is for my wife Samapti who has always been very understanding and encouraging. I appreciate being associated with such a nice family.

Finally, I thank God for providing me with all the basic amenities and making the texture of my life beautiful. 


\section{Contents}

1 Introduction 1

1.1 Brief Overview of Speech Coding Techniques . . . . . . . . . . 2

1.2 Utility of Objective Measure . . . . . . . . . . . . . 4

1.2.1 Evaluation of Coder Performance ............. 4

1.2.2 Rate-Distortion Analysis ............... 4

1.2.3 Design of Speech Coders ............... 5

1.3 Motivation for Our Research . . . . . . . . . . . . 6

1.4 Outline of the Thesis .................. 6

1.5 Our Contribution .................. 7

2 Distortion Measures for Speech Coding 9

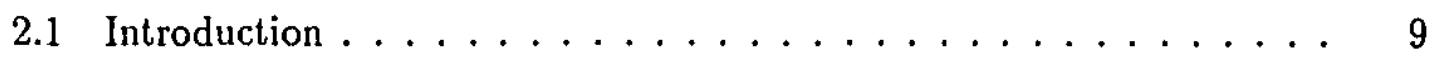

2.2 Subjective Quality Measures . . . . . . . . . . . . . . 10

2.2 .1 Utilitarian Tests . . . . . . . . . . . . . 10

2.2 .2 Analytic Tests ..................... 11

2.3 Time-Domain Objective Measures . . . . . . . . . . . . . 12

2.3.1 Signal-to-Noise Ratio . . . . . . . . . . . . . . 12

2.3.2 Segmental SNR . . . . . . . . . . . . . . 12

2.4 Spectral Objective Measures ................... 13 
$2.4 .1 \log$ Likelihood Ratio . . . . . . . . . . . . . . . 13

$2.4 .2 \log$ Area Ratio Xieasure $\ldots \ldots \ldots \ldots \ldots$

2.4.3 Line Spectral Frequency-based Measure . . . . . . . . . . 14

2.4.4 Log Spectral Distortion Measure . . . . . . . . . . 15

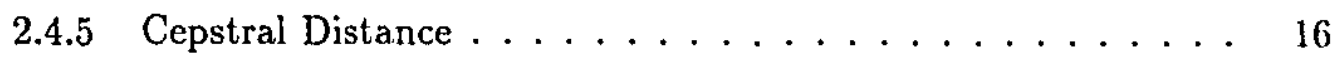

2.4 .6 Itakura-Saito Distortion Measure $\ldots \ldots \ldots \ldots \ldots$

2.4.7 Coherence Function . . . . . . . . . . . . . . . 17

2.5 Perceptually-Motivated Objective Measurcs $\ldots \ldots \ldots \ldots$. . . . . .

2.5 .1 Information Index $\ldots \ldots \ldots \ldots \ldots \ldots$

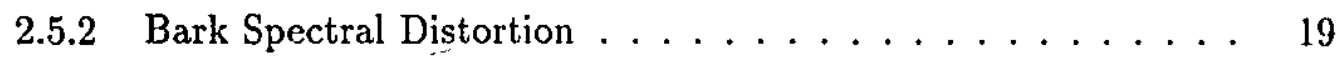

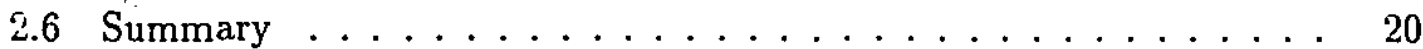

3 Auditory Representation of Speech 21

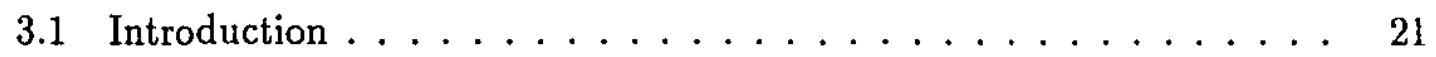

3.2 Mechanism of Auditory System $\ldots \ldots \ldots \ldots \ldots \ldots$

3.2.1 Ouer and Middle Ear $\ldots \ldots \ldots \ldots \ldots \ldots$

3.2 .2 Inner Ear (Cochlea) . . . . . . . . . . . . . . . 22

3.2.3 Inner and Outer Hair Cells . . . . . . . . . . . . . . 23

3.2 .4 Neural Pathways . . . . . . . . . . . . . 23

3.3 Psychoacoustic Observations . . . . . . . . . . . . . . . . 24

3.4 Perceptual-Domain Representation $\ldots \ldots \ldots \ldots \ldots$

3.4.1 Auditory Models for Speech Representation . . . . . . . 26

3.4.2 Mapping Using Lyon's Cochlear Model . . . . . . . . . . 28

3.4.3 Auditory Representation . . . . . . . . . . . . . . 34

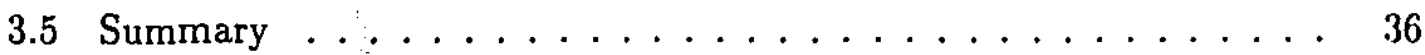


4 Cochleir Discrimination Information (CDI) Measure

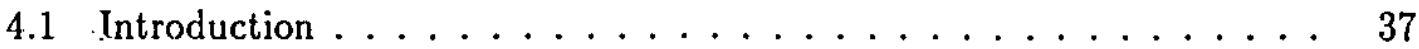

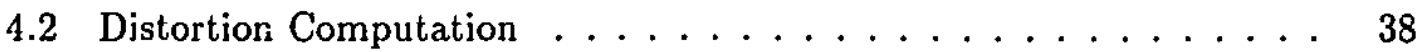

4.3 Experimental Results . . . . . . . . . . . . . . . . 41

4.3.1 Performance of Objective Measures . . . . . . . . . . 42

4.3.2 Effect of Different Entropies . . . . . . . . . . . . 46

4.3 .3 Effect of Gain Changes . . . . . . . . . . . . . . 46

4.3.4 Effect of Sample Delays _. . . . . . . . . . . 46

4.3.5 Speech Coder Identificatior . . . . . . . . . . . 48

4.4 Rate-Distortion Analysis . . . . . . . . . . . . . . . . 49

4.4.1 Preliminary Background .............. 50

4.4 .2 Kelevant Literature . . . . . . . . . . . . . . 51

4.5 Evaluation of Rate- Distortion Functicn . . . . . . . . . . 52

4.5.1 Source-Dustination Pair Characterizattion ......... 55

4.5.2 Calculation Pased on Blahut's Algorithm . . . . . . . . . . 54

4.5.3 Measured Performances of Speech Coders . . . . . . . . . 55

4.6 Summary . . . . . . . . . . . . . . . . . . 57

5 Cochlear Hidden Markuvian (CHírí) Measure 58

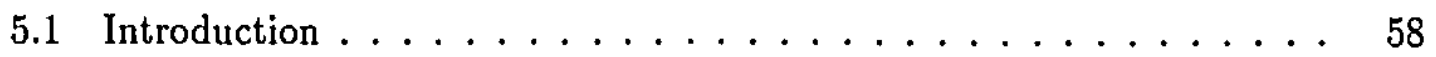

5.2 Characterization of Hidden Markov Model . . . . . . . . . . . . 59

5.3 Preliminaries ............................ 61

5.3.1 Forward and Backward Jikelihood Variables . . . . . . . 62

5.3 .2 Auxiliary Function .................. 64

5.4 Distortion Measure Methodology . . . . . . . . . . . . . 64 
5.4.i Parameter Estimation ............... 65

5.4 .2 Distortion Computation .............. 69

5.4 .3 Alternative Àpproaches . . . . . . . . . . . . 69

5.5 Practical Considerations ................ 71

5.5 .1 Computational Issues . . . . . . . . . . . 71

5.5.2 Initial Estimates for HMM Parameters .......... 71

5.5.3 Trairing Data and Iterations ............. 72

5.5.4 Mixture Frocesses ................... 72

5.6 Experimental Results . . . . . . . . . . . . . . . . 72

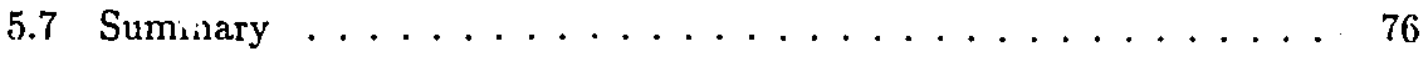

6 Applications in Coder Analysis $\quad \therefore 77$

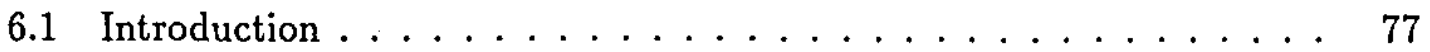

6.2 Existing Pitch Estimation Algorithms . . . . . . . . . . . 79

6.3 Pilch Frequency Estimation . . . . . . . . . . . . . 80

6.4 Wideband Coder Architecture . . . . . . . . . . . . 83

6.4.1 LSF-based Short-term Prediction .............. 84

6.4.2 Long-term Prediction with Fractional Delays . . . . . . . . 86

6.4.3 Residual Signal Codebook ............... 86

6.5 Perceptual Noise Weightirg . . . . . . . . . . . . . 87

6.5.1 Simple Noise Weighting . . . . . . . . . . . . . 88

6.5 .2 Codebook Shaping Iilter . . . . . . . . . . 89

6.5.3 Enhanced Noise Weighting . . . . . . . . . . 89

6.6 Performance Evaluation .................... 90

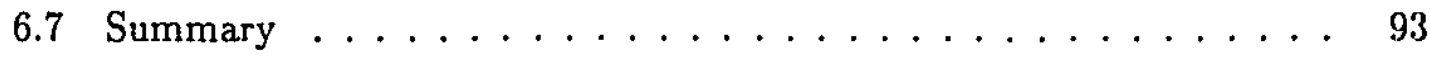


7 Concluding Remarks $\quad 94$

7.1 Surnmary of Our Work . . . . . . . . . . . . . . . . 94

7.2 Future Research Directions . . . . . . . . . . . . . . 97

7.2.1 Improvement of Model Structure $\ldots \ldots \ldots \ldots \ldots \ldots$

7.2.2 Reduction of Computational Complexity . . . . . . . . . 98

7.2.3 Administration of Formal Subjective Test $\ldots \ldots \ldots . \ldots 98$

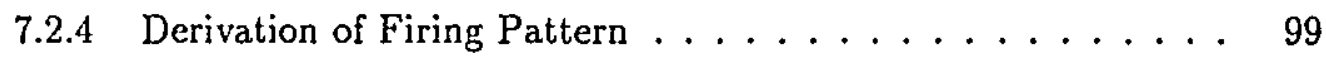

7.2.5 Application of Measures in Speech Coding . . . . . . . . 99

$7.3 \quad$ Epilog . . . . . . . . . . . . . . . . . . 100

$\begin{array}{ll}\text { Appendix } & 102\end{array}$

$\begin{array}{ll}\text { References } & 108\end{array}$ 


\section{List of Figures}

3.1 Block diagram of Lyon's cochlear model ('HWR' stands for the halfwave rectifier and 'AGC' stands for the automatic gain controller) . . 27

3.2 First-order outer-and-middle ear-filter . . . . . . . . . . . . 29

$3.3 s$-Domain pole-zero plots for typical stages (integrated notch and resonator filters $\ldots \ldots \ldots \ldots \ldots \ldots \ldots \ldots \ldots \ldots \ldots \ldots \ldots$

3.4 Bandwidths vs. center frequencics of sixty-four stages . . . . . 30

3.5 Initial stage filter $\ldots \ldots \ldots \ldots \ldots \ldots \ldots \ldots \ldots \ldots$

3.6 Magnitude responses for three typical ear-filter stages with $\int_{c}=499$; 1,013 and $2,509 \mathrm{~Hz} \ldots \ldots \ldots \ldots \ldots \ldots$

3.7 A typical automatic gain control (AGC) stage $\ldots \ldots \ldots \ldots . \ldots 34$

3.8 A typical steady-state response of four cascaded $A G C$ blocks . . . . 35

4.1 Time-domain waveforms and spectrograms of an original and three coded speech signals, "Oak is strong and also gives shade." . . . . . 43

4.2 The discrimination measure profiles $(J=2)$-(a) the dirceted divergence with $\alpha=1$ and (b) the directed divergence with $\alpha=2 . \quad \ldots \quad 47$

4.3 The discrimination measure profiles $(J=2)-(\mathrm{a})$ the $\chi^{2}$ divergence and $(\mathrm{b})$ the variational distance. $\ldots \ldots \ldots \ldots \ldots$

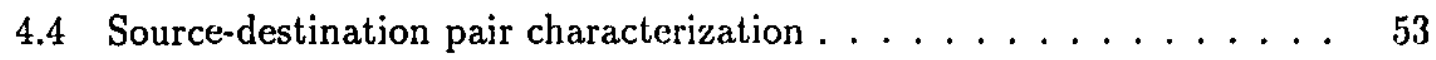


4.5 Speech coder rate in bits/sample vs, average cochlear variational distance measure (- - line shows an analytically derived lower bound, - line shows the exact rate-distortion curve using Blahut's algorithm and four '*' points [SC1-SC4] denote the performances of four speech coders $) \ldots \ldots \ldots \ldots \ldots \ldots$

4.6 Speech coder rate in bits/sample vs. average cochlear directed divergence (with $\alpha=1$ ) measure (— line shows the rate-distortion curve using Blahut's algorithm and four '*' points [SC1-SC4] denote the performances of four speech coders $) \ldots \ldots \ldots \ldots$

5.1 A two-state fully-connected hidden Markov model $\left(S_{0}\right.$ and $S_{1}$ denote the non-firing and firing states, $\pi_{0}$ and $\pi_{1}$ are the initial state probabilities, $a_{i j}$ gives the state transition probability from a state $S_{i}$ to a state $S_{j}, b_{0}(O)$ and $b_{1}(O)$ are the observation probability density functions for the state $S_{0}$ and $S_{1}$ respectively) . . . . . . . . . 60

5.2 A two-state trellis diagram ( $S_{0}$ and $S_{1}$ denote the non-firing and firing

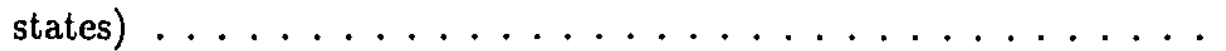

6.1 Time-domain waveform and spectrogram plot of the vowel /a/ in the word 'shade' (female voice) . . . . . . . . . . . . . . . .

6.2 One-dimensional cross-entropogram (directed divergence with $\alpha=1$ ) for one particular frame (160 samples starting from the sample number $15,000)$ of $/ a /$ in the word 'shade' . . . . . . . . . . . . 83

6.3 Noise weighting with $\gamma=0.75 \ldots \ldots \ldots 8$

6.4 Noise level using codebook shaping filter. . . . . . . . . . . 89

6.5 Performance of the weighting filter with $N=2$ and $\delta=0.7 \ldots \ldots 0$ 


\section{List of Tables}

4.1 Different measure values for three coded signals (with threc different $4.8 \mathrm{kbps}$ speech coders) with reference to the original spcech utterance F3 (' $x$ ' indicates that the objective measures for 'oakf8'' and 'oak $88 \mathrm{k}$ ' do not agree with the subjective rankings) $\ldots \ldots \ldots \ldots \ldots 44$

4.2 Subjective and objective measure values for coded signals with reference to the corresponding original speech utierances (M1-M6 (male) and $\mathrm{F} 1-\mathrm{F} 6$ (female) are speech utterances, $\mathrm{C} 1-\mathrm{C} 6$ are specch coders, ' $S$ ' denotes the average subjective ranking scores and ' $D_{1}$ ' gives the directed divergence measure values with $\alpha=1) \ldots \ldots \ldots$

4.3 The directed divergence (with $\alpha=1,2$ ) measure values with zero, onc, two and three sample delays for the coded signal 'oakf8f' and 'oakf8k' with reference to the original speech sentence $\ldots \ldots \ldots \ldots$

4.4 Speech coder identification for two sentences M1 and F3 (the sample numbers played and the fraction of listeners who have correctly identified the coders are provided in the table) $\ldots \ldots \ldots \ldots$

5.1 Subjective and objective measure values for six coded signals with reference to the corresponding original speech utterances (" $S$ " gives the average subjective ranking scores and ' $H$ ' denotes the cochlear hidden Markov measure with single channel (CHM-SC)) . . . . . . .

5.2 Subjective and objective measure values for six coded signals with reference to the corresponding original speech utterances (' $S$ ' gives the average subjective ranking scores and ' $H$ ' denotes the cochlear hidden Markov measure with three channels (CHM-TC)) . . . . . . 
5.3 The SNR and the cochlear hidden Markovian-three channels (CHMTC) measure values with zero, one, two and three sample delays for the coded signal 'oakf8f' and 'oakf8k' with reference to the original speech sentence .....................

6.1 Distortion measures for different noise weighting configurations (the segmental $\mathrm{SNR}$ values $\left(\mathrm{SNR}_{\text {seg }}\right)$, the cochlear discrimination information measure values with $\alpha=1$ (CDI) and the cochlear hidden Markovian measure with three channels (CHM-TC) are tabulated) . . . . 


\section{Chapter 1}

\section{Introduction}

In a typical source coding problem, a continuous-time continuous-amplitude bandlimited signal is sampled in the time domain at or above the minimum sampling rate required. This time-discretized signal with amplitude having continuous probability density function has an infinite entropy. To transmit the output of such a source and recover it exactly, a communication channel of infinite capacity is required. In practice, every channel, due to perturbation by noise, has a finite capacily. Thus, it is not possible to transmit the output of a continuous source over any channel and recover it exactly [1]. Accepting the fact that there will inevitably be some distortion, a typical source coder minimizes it by removing deliberately some information which is deemed 'not very important' to the destination. 'The extent to which the information should be removed depends on the bit-rate of the coder; the lower the bit-rate, the more information is needed to be removed.

In speech communication, the ultimate recipient of information is a human being and hence his/her perceptual abilities govern the precision with which speech data must be processed and transmitted. Thus, to reduce the amount of distortion, the speech data can be modified by an intentional removal of some information in accordance with the limitations of the auditory system. Determining 'what is not very important' to the auditory system and 'how the auditory system assesses' the relative importance of information is the primary task involved in devising a distortion measure for speech coders.

The sound quality of a given speech coder can best be evaluated by listening to 
it. However, an extensive subjective testing of speech coders is difficult to administer and also time-consuming. Often, it is found to give inconsistent result due to the inherent non-repeatability of human responses. Moreover, it does not provide much insight into the factors which may lead to an improvement of the speech coding system. It is obvious that an objective quality measure, if suitably defined, could play an important role in the evaluation as well as in the design of low bit-rate speech coders. One important advantage of an objective quality measure is that its repeated application at different time under different environment gives the same performance.

Defining an appıupriate objective quality measure for coded/distorted speech has thus become one of the pressing tasks to maintain a 'good' speech quality with low bit-rate coding or to assess the perceptual quality of any speech coder. We provide a brief overview of speech coding techniques in Section 1.1. The utility of having a 'good' objective measure is discussed in Section 1.2 and the motivation for our research is explained in Section 1.3. We present an outline of the thesis in Section 1.4 and state our contribution to original knowledge in Section 1.5.

\subsection{Brief Overview of Speech Coding Techniques}

The primary objective in speech coding research is to determine strategies for generating synthesized signals with as high quality as possible and at the same time adhering to other constraints such as bit-rate and coding delay. Many coding techniques exist for rates starting from 64 kilobits per sec (kbps) all the way down to $2.4 \mathrm{kbps}$ (or even lower). Speech coding algorithms vary from the high-rate/lowcomplexity waveform coders to the medium- to low-rate/high-complexity vocoders or hybrid coders [2]. Their variations are primarily in the following four aspects: (i) selection of information (features) to be encoded, (ii) representation of features by appropriate parameters for encoding, (iii) quantization technique adopted for parameter discretization and (iv) distortion measures applied for estimating quantization loss.

Waveform coders analyze, code and reconstruct speech on a sample-by-sample basis. Time-domain waveform coders exploit waveform redundancies, e.g., the periodicity and slowly varying intensity while the spectral-domain waveform coders take advantage of the nonuniform distribution of speech information across different fre- 
quencies [3]. On the other hand, source coders or vocoders utilize speech-specific model. They generally identify certain aspects of the speech spectrum as being important to model and generate speech with good reproduction of these aspects $[4]$. In speech production, the source may be either periodic generating a voiced speech or noisy and aperiodic resulting in an unvoiced speech. The fundamental frequency of the vocal cord vibration, in the utterance of a voiced speech, is termed as the pitch frequency. The resonances, termed as the formants, occur due to the poles of the vocal tract frequency response while the spectral nulls (anti-resonances) occur duc to its zeros. Currently, the code-excited linear prediction (CELP) algorithm is the most widely used speech coding method and a typical low or medium bit-rate such coder encodes the formant, pitch and residual information separately [5].

An all-pole linear prediction filter synthesizes formant information and the filter parameters are determined by autocorrelation or covariance method. These parameters may be encoded directly or may be expressed in other forms such as reflection coefficients, area ratio parameters, line spectral frequency parameters etc. and then coded. They differ from each other from the perspectives of computational efficiency, quantization sensitivity etc. [2]. A pitch prediction filter is generally characterized by the pitch gain and lag value parameters. Depending on the bits available, the number of pitch predictor taps and the codebook size for the pitch parameters are decided [6]. For sending information about the residual signal, a random excitation codebook or often a structured algebraic codebook is used [7] and an appropriate codebook entry is selected from it.

While designing a speech coder, once the parameters pertaining to different features are appropriately selected, they are quantized. The quantization may be scalar, vector (single- or multi-stage) and scalar-vector. For example, U.S. federal standard $4.8 \mathrm{kbps}$ CELP coder [5] has formant filter with scalar quantization of line spectral frequency parameters. However, vector quantization provides a substantial bit reduction for the same speech quality at the expense of higher memory and computational complexity. Different variations and hybrid forms of scalar and vector quantizations are used for different coder rates [8]. Similarly, the pitch prediction parameters can also be quantized in various ways [9]. The residual signals are usually vector-quantized and stored in a stochastic codebook [5].

In an analysis-by-synthesis type speech coder, the selection of appropriate indices for different codebook entries requires minimizing a distortion criterion. It is 
possible to define different types of distortion measures for different features. However, selecting a codeword with respect to a global distortion measure could yield better results as such a selection could even take care of interactions among the features. The overall perceptual quality of a given speech coder could be evaluated subjectively or by a properly defined objective measure. In this research, we have primarily concentrated on the formulation of distortion measures using an auditory model in the front-end. We have not attempted to use the measure in the coding process, but only in the evaluation of speech coder performances.

\subsection{Utility of Objective Measure}

In this section, we explain the utility of deriving an objective quality measure. Obtaining a suitable distortion measure could offer several advantages such as (a) its use in evaluating speech coder performances, (b) its application in a rate-distortion analysis which could indicate the lowest possible bit-rate required for a particular speech quality and (c) its use in the design procedure of speech coders.

\subsubsection{Evaluation of Coder Performance}

Speech coders of several standardized data rates are designed to 'match' to the capacities of different communication channels. These encoders vary from each other from the view point of the coder architecture, the type of features encoded, the number of bits allocated to the features and so on. A wide variety of encoding algorithms introduces a broad range of linear and nonlinear coder distortions. All of these distortions are not equally perceived by the auditory system. As a consequence, if we can devise a distortion measure incorporating the human perception procedure, then that can appropriately be used to evaluate the performances of different speech coders.

\subsubsection{Rate-Distortion Analysis}

The need for a strong mathematical foundation for the field of data compression has resulted in the development of rate-distortion theory. The performance achieved by various data compression systems can be compared with absolute bounds derived from 
rate-distortion theory. With a particular source and a defined distortion measure, it is possible to draw a rate-distortion curve which determines the lowest possible rate for allowing a particular amount of coder distortion. However, if the distortion measure is not properly defined, this limit may not portray the real picture. Defining an appropriate distortion measure would facilitate the determination of the coder rate limit for attaining a particular speech quality.

\subsubsection{Design of Speech Coders}

A distortion measure can help the design procedure of speech coders in three ways:

(1) In an analysis-by-synthesis type speech coder, from a stochastic codebook, all innovation sequence entries (in the case of an 'optimal' coder) or selectively chosen entries (in the case of a 'suboptimal' coder) are used along with the formant and pilch synthesis filters to generate several coded speech signals. Finally, the index of that codebook entry is transmitted which results in the minimum distortion as measured by the defined fidelity criterion. The distortion measure can thus be instrumental in selecting an 'appropriate' codebook entry.

(2) With a limited number of bits available per second, a strategic allocation of bits to different feature parameters becomes very important. The bit allocation strategy adopted for an $8 \mathrm{kbps}$ coder can neither be scaled down directly for a $4 \mathrm{kbps}$ coder nor be scaled up for a $16 \mathrm{kbps}$ coder. The relative importance of the information to be transmitted plays a significant role. In the design phase, the defined distortion measure can be used for improving the bit allocation policy of a particular speech coder, be it a waveform coder, an analysis-by-synthesis coder or a vocoder.

(3) While designing a speech coder, an appropriate distortion measure not only helps in making a sound bit allocation policy, but also in 'populating' (also called 'training') the codebook. In the training phase, determining the centroid for each class with the defined distortion measure results in the design of an 'optimum' (at least in the local sense) codebook. If the distortion measure properly reflects the perceptual importance of information, then a fixed size codebook designed in this way will also be filled up with the entries which contain perceptually important information. 


\subsection{Motivation for Our Research}

Over the last two decades, several objective measures have been suggested in the literature (references are in Chapter 2). It is a well-known fact that the time-domain objective measures such as the signal-to-noise ratio and the segmental signal-to-noise ratio do not perform well in the assessment or in the design of a low or medium bit-rate speech coder. Spectral measures, e.g., the log likelihood ratio measure, the log area ratio measure, the log spectral distortion measure and the Itakura-Saito measure, exhibit a better performance. However, most of these measures are based on the parameters of linear prediction filter modeling the formant structure and thus do not adequately feature the perceptual phenomena. Only about $80 \%$ of the perceived degradation in speech quality can be explained by the distortions of the spectral peaks or speech formants [10]. Therefore, it is important for a 'good' quality measure to consider distortion not only in the formant information, but also in the pitch and the residual information.

A few psychoacoustically-motivated measures such as the information index and the Bark spectral distortion measure has also bein studied. In the recent literature, several auditory models have been proposed and investigated (references are in Chapter 3). Some of these models emulate the psychoacoustic observations fairly well, at least at the level of auditory periphery. Thus, an application of one such auditory model in the formulation of a distortion measure could result in good performance. This may, to some extent, increase the complexity of computing the measure value. Nevertheless, we believe that the measure could be used widely in practice as the computational burden is eased with further progress in the signal processing technique and the VLSI technology. Keeping this view in mind, we have conducted research on the topic of Auditory Distortion Measures for Speech Coder Evaluation. We emphasize accuracy over computational considerations in the evaluation of speech coders.

\subsection{Outline of the Thesis}

The format of the dissertation is as follows. Chapter 2 reviews the existing timedomain, spectral-domain as well as perceptually-motivated distortion measures. Chapter 3 discusses psychoacoustic observations relevant to speech perception, describes 
Lyon's auditory (cochlear) model and defines a perceptual-domain. Chapter 4 proposes a cochlear discrimination information measure which compares the set of perceptual-domain parameters for the original and the coded speech signals. With this measure, performance of several speech coders is evaluated objectively and also a ratedistortion-theoretic analysis is pursued. Chapter 5 puts forward another distortion measure methodology which uses hidden Markov models. This measure is computationally more intens've, but captures the basics of high-level processing in addition to the signal processing at the auditory periphery. Chapter 6 outlines some other applications of the measures, for example, in the pitch extraction or in the evaluation of perceptual weighting schemes usually incorporated in a specch coder. Chapter 7 concludes this dissertation with relevant remarks and future research directions.

\subsection{Our Contribution}

In this thesis, we consider an auditory model and suggest two distinct approaches for devising distortion measures for coded speech. The fundamental difference between our approaches and the existing perceptually-motivated measures is in addressing the issue of the 'cause' rather than that of the 'effect' involved in speech perception. In other words, instead of merely considering the important perceptual effects observed, we emulate the auditory system as it is and use it in the formulation of our distortion measure.

Our primary contribution is in the processing of neural information obtained at the output of Lyon's auditory model. As explained in the dissertation, in reality, a series of electrical spikes (firings) is transmitted from the auditory periphery to the brain through the neural pathways. Here, we treat the neural pathways to be communication channels with an input alphabet of size two, i.e., firing and non-firing. Our first distortion measure deals with the neural firing probabilities and evaluates the neural firing cross-entropy of the coded speech with respect to that of the original one. With this measure, we compute the rate-distortion function for speech coder determining the lowest bit-rate required for a given amount of distortion. Spech coders with rates ranging from $4.8 \mathrm{kbps}$ to $32 \mathrm{kbps}$ are studied from the viewpoint of their performance with respect to the rate-distortion limits. In the second measure, a two-state (one each for firing and non-firing events) fully-connected hidden Markov model (HMM) is associated with each of the neural channels and various model 
parameters are derived with the pertinent neural firing information of the original signal. For computing the coder distortion, the neural firing observations from the coded speech are matched against the derived HMMs. We believe that the second measure is more powerful as it utilizes the contextual information present in the neural firing pattern. Experimental results show that these measures conform to subjective evaluation results in majority of the cases. The introduced measures are also applied in speech coder analysis, e.g., in the pitch frequency determination and the evaluation of noise weighting schemes usually incorporated in a low bit-rate coder. 


\section{Chapter 2}

\section{Distortion Measures for Speech Coding}

\section{$2.1 \quad$ Introduction}

A distortion measure for speech quality is a measure which can be compiuted directly from an original speech waveform and its coded/distorted version; and which conforms to the results of a subjective measure of speech quality [11]. Regression analysis establishes a quantitative relationship between an objective quality measure and a subjective evaluation method. A correlation coefficienl $(\rho)$, defined as [12]

$$
\rho=\frac{\sum_{k}\left(S_{k}-\bar{S}\right)\left(O_{k}-\bar{O}\right)}{\left[\sum_{k}\left(S_{k}-\bar{S}\right)^{2}\right]^{1 / 2}\left[\sum_{k}\left(O_{k}-\bar{O}\right)^{2}\right]^{1 / 2}},
$$

is often used as a figure-of-merit to measure the degree of correlation between a standard subjective measure and an objective measure. In (2.1), $S_{k}$ and $O_{k}$, respectively, are the subjective and objective measure values for the $k$-th speech utterance in a particular database; and $\bar{S}$ and $\bar{O}$ are the corresponding average test. scores, averaged over all the utterances of the database. One major disadvantage of applying the regression analysis technique is the necessity of knowing the form of the regression equation a priori. An alternative method [13] uses Bayesian estimation and a nonlinear relationship is automatically determined during the training.

Several subjective as well as objective measures have been proposed in the litcrature. For many such subjective and objective measure pairs, the degrees of correlation 
have also been determined. In Section 2.2, some of the standard subjective evaluation procedures are outlined. We Lescribe a major class of time-domain distortion measures in Section 2.3 and a few spectral distortion measures in Section 2.4. Some of the perceptually-motivated distortion measures are discussed in Section 2.5.

\subsection{Subjective Quality Measures}

Subjective quality measures can be classified into two primary categories [14]: utilitarian and analytic. The utilitarian quality measures are found to be reliable and reasonably efficient in the test administration. These measures are based on a unidimensionai scale and the result is provided by a single number so that the coded speech qualities can be directly compared. On the other hand, the analytic measures typically use more than one dimension for assessing the speech quality and are directed towards characterizing the underlying psychological components that determine the perceived quality. With either of the classes, an extensive listener training procedure is needed to ensure the reliability of these tests under different test environments.

\subsubsection{Utilitarian Tests}

Subjective measures very often address the speech intelligibility and the articulation aspects separately. The intelligibility tests are scored by the percentage of correct understanding of the meaning conveyed by the transmitted speech while the articulation tests are evaluated by the percentage of correct recognition of the sounds, words or sentences. Fletcher and Steinberg [15] have constructed, for their articulation test, random lists of nonsense monosyllables (nullifying the associated semantic memory) in the form of consonant-vowel-consonant (C-V-C). Later, Fairbanks [16] has modified this test by specifying the trailing vowel-consonants to the listeners and asking them to choose the leading consonant based on his/her interpretation of the test speech. Many refined versions of such rhyme tests have subsequently been suggested where the listener responses are restricted in different manners (e.g., the choice being limited to a finite set of rhyming words).

These tests are found to be appropriate for speech coding systems that generate moderately to severely distorted speech [11]. However, for highly intelligible systems, 
other perceivable attributes (e.g., pleasantness, naturalness) become important. In an isopreference evaluation procedure [17], test speech signals each having a different speech level and contaminated with different levels of additive noise are passed through the test transmission system. Test results are usually reported as isopreference contours' in the two-dimensional parameter space of speech level vs. noise level. Listeners usually judge the test: speech in terms of a reference spcech; hence, they are often compelled to consider a smaller perceptual descriptor space than that might be desired. The most widely used utilitarian type subjective evaluation method is the mean opinion score (MOS) [18] in which the listeners rate the speech distortion under test on a five.point absolute scale (Rate 5: imperceptible; Rate 4: just perceptible, but not annoying; Rate 3: perceptible and slightly annoying; Rate 2: annoying, but not objectionable; Rate 1: very annoying and objectionable). Since the listeners have freedom to interpret the scale 'ratings' in their own way, the MOS score provides an agglomerative measure value for different types of coder distortions.

\subsubsection{Analytic Tests}

An alternative subjective evaluation approach is to rate the test specch on a multidimensional scale. One such popular multidimensional measure is the diagnostic acceptability measure (DAM) [19]. The DAM evaluates a spcech signal on sixtecn separate scales (covering the signal quality, the background quality and the overall quality), all of which have a range from 0 to 100 points. In a multidimensional perceptual space, the distorted speech signals are represented as points so that the relationship between an individual preference and an acoustic distortion can be studied [20]. Signal degradations such as jiuttering (amplitude-modulated specch), thin (high-pass speech), rasping (peak-clipped speech), interrupted (packetized speech with 'glitches'), nasal; background noise such as hissing (nojse-masked speech), buzzing (tandemmed digital systein), babbling (narrowband system with errors), rumbling (low-frequency noise-masked speech); and overall qualities such as intelligibility, pleasantness, acceptability are considered in the DAM test [11]. This measure attempts to minimize the errors involved in the measurement process as well as that associated with the human variability. 


\subsection{Time-Domain Objective Measures}

The most popular class of the time-domain measures is the signal-to-noise ratio (SNR) with its varied forms (e.g., the segmental SNR, the granular segmental SNR).

\subsubsection{Signal-to-Noise Ratio}

The signal-to-noise ratio (SNR), for measuring the coded speech quality, is defined as

$$
\mathrm{SNR}=10 \log _{10} \frac{\sum_{n} x^{2}[n]}{\sum_{n}(y[n]-x[n])^{2}} \mathrm{~dB},
$$

where $x[n]$ and $y[n]$ are the $n$-th original and coded speech samples, respectively. Numerous studies [11] have exhibited that the SNR measure does not correlate well with subjective evaluation results. In practice, any phase distortion with a delay variation limited to a few milliseconds has such a smail effect on the signal quality that it can be disregarded in the context of most synthetic speech quality [21]. However, the SNR measure degrades drastically with any time misalignment of $\{x[n]\}$ and $\{y[n]\}$. The correlation coefficient (with the MOS score) for the SNR measure has been found to be 0.24 correlated only across the waveform-coder distortions [11] where it is expected to perform relatively well.

\subsubsection{Segmental SNR}

A major drawback of the SNR measure is that it treats the entire speech utterance as a single vector thereby presuming an unrealistic idea of a single comparison made by the listener after listening to the entire utterance. A better measure, usually referred to as the segmental SNR ( $\mathrm{SNR}_{\text {seg }}$ ), is an average measure of the SNR values in $\mathrm{dB}$. The averaging is done over the $M$ speech 'segments' present in an utterance, each segment being of the order of $16 \mathrm{~ms}$ long (i.e., $N=128$ samples with $8,000 \mathrm{~Hz}$ sampling rate). Mathematically, this measure can be written as [22]

$$
\mathrm{SNR}_{\text {seg }}=\frac{10}{M} \sum_{m=0}^{M-1} \log _{10}\left\{\frac{\sum_{n=1}^{N} x^{2}[n+N m]}{\sum_{n=1}^{N}(y[n+N m]-x[n+N m])^{2}}\right\} \mathrm{dB} .
$$


The correlation coefficient associated with this measure has been determined to be 0.77 across a wide range of waveform coder distortions [11]. Though SNR seg $_{\text {provides }}$ better accuracy than the SNR measure, it also can not be considered as a reliable measure for the speech quality. In segments where an original speech has almost no sirnal components, a little noise could give rise to a large negative SNR for that segment which in turn causes a considerable bias in the overall measure of $\mathrm{SNR}_{\text {seg }}$. A threshold-adjusted or frequency-weighted SNR $_{\text {seg }}$ measure could be used which alleviates this problem to a great extent [11]. Another variation of the $\mathrm{SNR}_{\text {seg }}$ measure is the granular $\mathrm{SNR}_{\text {seg }}$ which has been found to be appropriate only for the evaluation of delta modulation or differential waveform coders [23].

\subsection{Spectral Objective Measures}

Several spectral distortion measures have been proposed in the literature including the log likelihood ratio measure, the log area ratio measure, the line spectral frequencybased measure, the log spectral distortion measure, the cepstral distance, the ItakuraSaito measure and the coherence function. These distortion mcasures arc gencrally computed using speech segments typically between 15 and $30 \mathrm{~ms}$ long. They are much more reliable than the SNR measure and are less sensitive to the occurrence of time misalignments between the original and the coded specch [11].

\subsubsection{Log Likelihood Ratio}

The log likelihood ratio (LLR) distance for a speech segment is defined based on the assumption that samples of a speech can be represented by a $p$-th order all-pole linear predictive coding (LPC) model of the form

$$
x[n]=\sum_{m=1}^{p} a_{m} x[n-m]+G_{x} u[n],
$$

where $x[n]$ is the $n$-th speech sample, $a_{m}$ (for $m=1,2, \cdots, p$ ) are the coefficients of an all-pole filter $1 / A_{x}(z)$ which models the resonances of the speech production mechanism, $G_{x}$ is the gain of the filter and $u[n]$ is an appropriatc excitation source 
for the filter. The LLK measure then can be defined as [24]

$$
\mathrm{LLR}=\log \left[\frac{a_{x} R_{y} a_{x}^{T}}{a_{y} R_{y} a_{y}^{T}}\right]
$$

where $a_{x}$ is the LPC coefficient vector $\left[1,-a_{1}^{x},-a_{2}^{x}, \cdots,-a_{p}^{x}\right]$ for the original speech $\{x[n]\}$ and $a_{y}$ is the LPC coefficient vector $\left[1,-a_{1}^{y},-a_{2}^{y}, \cdots,-a_{p}^{y}\right]$ for the coded speech $\{y[n]\} . \boldsymbol{R}_{y}$, the correlation matrix of $\{y[n]\}$, has elements as

$$
r_{y}(i, j)=\sum_{n=1}^{N-|i-j|} y[n] y[n+|i-j|], \quad \text { for } i, j=0,1, \cdots, p,
$$

where $N$ is the number of samples used in the analysis. The denominator in (2.5) measures the prediction residual energy when $\{y[n]\}$ is filtered with its all-zero analysis filter $A_{y}(z)$, whereas the numerator measures the same when $\{y[n]\}$ is passed through the filter $A_{x}(z)$. A correlation coefficient of 0.59 is achieved with this measure [11].

\subsubsection{Log Area Ratio Measure}

The reflection coefficients $\left\{k_{m}\right\}$, another representation of the LPC coefficients $\left\{a_{m}\right\}$, are spectrally less-sensitive to quantization than their counterparts. However, the reflection coefficients can be sensitive to quantization errors when their magnitudes are near unity (i.e., they represent narrow-bandwidth poles). To reduce the sensitivity, a suitable nonlinear transformation expanding the region near $\left|k_{m}\right|=1$ can be followed based on which a log area ratio (LAR) distortion measure is defined as [11]

$$
\mathrm{LAR}=\sum_{m=1}^{p}\left[\log \left(\frac{1-k_{m}}{1+k_{m}}\right)-\log \left(\frac{1-k_{m}^{\prime}}{1+k_{m}^{\prime}}\right)\right]^{2},
$$

where $p$ is the number of predictor coefficients and $k_{m}, k_{m}^{\prime}$ (for $m=1,2, \cdots, p$ ) are the reflection coefficients corresponding to the original and the coded signals, respectively. A correlation coefficient 1 t the order of 0.62 is attained with this measure [11].

\subsubsection{Line Spectral Frequency-based Measure}

The line spectral frequency (LSF) coefficients are derived by mapping the $p$-zeros of an all-zero analysis LPC filter $A(z)$ onto the unit circle through two orthogonal 
polynomials $P(z)$ and $Q(z)$ of $(p+1)$-st order as [2]

$$
\begin{aligned}
& P(z)=A(z)+z^{-(p+1)} A\left(z^{-1}\right) \\
& Q(z)=A(z)-z^{-(p+1)} A\left(z^{-1}\right) .
\end{aligned}
$$

The resulting polynomials, $P(z)$ and $Q(z)$, have their roots in conjugate pairs. A multiobjective functional measure is formulated by using the LSF transformation in determining the spectral peak locations and the spectral peak bandwidths for the original and the distorted speech frames. This measure compares six parameters which are (a) a shift in peak location, (b) a change in peak bandwidth, (c) a change in peak energy, (d) differences in inter-frame peak movement, (c) lost peaks and ( $f$ ) distortion-induced extra peaks. This measure has exhibited a correlation coefficient of $0.78[10]$.

\subsubsection{Log Spectral Distortion Measure}

The notions of one-step prediction error and spectral factorization are two important properties using which an $L_{p}$ norm-based log spectral distortion (LSD) measure is defined between two log spectral densities as [25]

$$
\operatorname{LSD}=\left\{\int_{-\pi}^{\pi}|V(\omega)|^{p} \frac{d \omega}{2 \pi}\right\}^{1 / p},
$$

where

$$
V(\omega)=\log \left[\frac{G_{x}^{2}}{\left|A_{x}\left(e^{j \omega}\right)\right|^{2}}\right]-\log \left[\frac{G_{y}^{2}}{\left|A_{y}\left(e^{j \omega}\right)\right|^{2}}\right]
$$

with $G_{x}$ and $G_{y}$ as the LPC gain coefficients; and $A_{x}\left(e^{j \omega}\right)$ and $A_{y}\left(e^{j \omega}\right)$ as the LPC model polynomials corresponding to the original and the coded speech signals, respectively. The most common choices in (2.10) for $p$ are 1,2 and $\infty$ giving rise to the mean absolute, the root mean square and the maximum deviation, respectively. A computational form of frequency-weighted log spectral distortion (FWLSD) measure is often given as

$$
\text { FWLSD }=\left\{\frac{\sum_{\nu=1}^{M}|X(\nu)|^{\gamma}|20 \log X(\nu) / Y(\nu)|^{p}}{\sum_{\nu=1}^{M}|X(\nu)|^{\gamma}}\right\}^{1 / p},
$$


where $M$ is an integer corresponding to $M$-point discrete Fourier transform (DFT), $\nu$ is the discrete frequency variable; and $X(\nu)$ and $Y(\nu)$ are the LPC spectra of $\{x[n]\}$ and $\{y[n]\}$, respectively. With $p=2$ and $\gamma=0.5$, a magnitude correlation coefficient of 0.60 is obtained [11]. Another version of this measure has recently been proposed in [26] where the kernel of the measure is not the original and the coded signal spectra, but the coded signal spectrum and the spectral representation of the nonlinear distortions incurred in the coding process.

\subsubsection{Cepstral Distance}

The basic problem with the LSD measures is the Fourier transform and logarithm computations involved in obtaining sufficient values of $V(\omega)$ in order to approxirnate the integral of (2.10) by summation. Computational efficiency and a high correlation with the root mean square LSD have thus made another measure, namely the cepstral distance (CD), popular [27]. The CD is a measure of the overall difference between an original and a corresponding coded speech cepstra. A cepstrum computed from the LPC coefficients, unlike that computed directly from the speech waveform, results in an estimate of the smoothed speech spectrum [28]. This can be written as

$$
\log \left\{\frac{1}{A(z)}\right\}=\sum_{k=1}^{\infty} c[k] z^{-k}
$$

where $A(z)$ is the LPC analysis filter polynomial and $c[k]$ denotes the $k$-th cepstral coefficient. Accordingly, a CD measure is defined as

$$
\mathrm{CD}=\frac{10}{\log 10} \sqrt{2 \sum_{k=1}^{L}\left(c_{x}[k]-c_{y}[k]\right)^{2}},
$$

where $c_{x}[k]$ and $c_{y}[k]$ are the $k$-th cepstral coefficients of the original and the distorted specch, and $L$ is the number of the cepstral coefficients used. Although the sequence of the cepstral coefficients is infinite in (2.13), limiting it to three times the number of LPC parameters shows almost no deterioration in the result. A correlation coefficient of 0.80 has been obtained with this measure [11]. The quefrency-weighted CD [29], the liftering window-based $\mathrm{CD}[30]$ are some examples of weighted CD measures. A unifying framework for viewing different distortion measures in the cepstral domain has been laid out in [31]. 


\subsubsection{Itakura-Saito Distortion Measure}

With a maximum likelihood formulation of linear prediction, Itakura and Saito have defined a $d_{1 \mathrm{~S}}$ measure as [32]

$$
d_{\mathrm{IS}}=\frac{1}{2 \pi} \int_{-\pi}^{\pi}\left[e^{V(\omega)}-V(\omega)-1\right] d \omega,
$$

where $V(\omega)$ is as given in (2.11). The assumptions used in deriving the integral form of (2.15) are that the speech is generated by a Gaussian process, the result of uncorrelated noise passed through an all-pole LPC filter and that the analysis interval is much longer than the all-pole filter order. It has been shown in [25] that the $d_{\mathrm{IS}}$ measure is twice the asymptotic discrimination information under the above assumption. A frequency-weighted version of the Itakura-Saito measure has been found in [33] to give a better performance. The $d_{\text {cosh }}$ measure, a symmetrical version of $d_{\mathrm{IS}}$; is often defined as

$$
d_{\mathrm{cosh}}=\frac{1}{2 \pi} \int_{-\pi}^{\pi}\{\cosh [V(\omega)]-1\} d \omega .
$$

It has been found in [27] that the $d_{\text {cosh }}$ measure bounds the LSD measure from above, and in [25] that it becomes one half of the generalized Ornstein distance between two Gaussian processes. Computational costs for evaluating the $d_{\mathrm{IS}}$ and $d_{\text {cosit }}$ measures are given in [34].

\subsubsection{Coherence Function}

In this method, the speech frames are first divided into four groups based on the four amplitude quartiles. The original and the coded signal power spectra as well as the cross-power spectrum are computed and averaged for all the frames in each quartile. The respective average spectra, denoted by $S_{x x}(f), S_{y y}(f)$ and $S_{x y}(f)$, are used to compute the squared coherence function $\gamma^{2}(f)$ as [35]

$$
\gamma^{2}(f)=\frac{\left|S_{x y}(f)\right|^{2}}{S_{x x}(f) S_{y y}(f)}
$$

which can be interpreted as the correlation between the original and the coded signals at a frequency $f$. Next, the signal power $C(f)$ and the distortion power $D(f)$ are 
estimated from $\gamma^{2}(f)$ and used to develop a modified signal-to-distortion ratio (SDR) for each quartile as

$$
\begin{aligned}
C(f) & =\eta \cdot \gamma^{2}(f)\left|S_{y y}(f)\right|^{2} \\
D(f) & =\eta \cdot\left[1-\gamma^{2}(f)\right]\left|S_{y y}(f)\right|^{2} \\
\mathrm{SDR} & =\frac{C(f) \cdot W_{2}(f)}{D(f) \cdot W_{2}(f)+W_{1}(f)}
\end{aligned}
$$

In (2.18) and (2.19), $\eta$ is a scale factor. $W_{1}(\bullet)$ and $W_{2}(\bullet)$ in $(2.20)$ are the weighting functions related to the hearing threshold and the handset receiver sensitivity. The regression-analyzed MOS value is calculated using a frequency-weighted quartileweighted nonlinear function; the details are given in [35].

\subsection{Perceptually-Motivated Objective Measures}

Coder distortions can be perceived if the magnitude of the distortion is greater than the resolution of the human auditory system. The nature of the distortion is also important from the perception point of view. In the following, we discuss two perceptually-motivated distortion measures.

\subsubsection{Information Index}

An information index (II) measure which accounts for loss, noise and distortion in speech transmission over a telephone network has been proposed in [36]. The auditory system effect is roughly modeled by dividing the spectrum into sixteen critical bands and applying empirical frequency weights and hearing thresholds for each band. At first, a signal-to-distortion ratio for each critical band, denoted as $R(i)$, is computed by

$$
R(i)=10 \log _{10} \frac{\sum_{j \in b_{i}}\left|X\left(\omega_{j}\right)\right|^{2}}{\left.\left|\sum_{j \in b_{i}}\right| X\left(\omega_{j}\right)\right|^{2}-\sum_{j \in b_{i}}\left|Y\left(\omega_{j}\right)\right|^{2} \mid} \mathrm{dB},
$$

where $j$ ranges over all the frequencies specified for the $i$-th band $b_{i}$. Here, $X(\omega)$ and $Y(\omega)$ are the Fourier transforms of an original and a corresponding coded speech 
frame. Assuming the bands to be independent, the II measure is computed as

$$
\mathrm{II}=\sum_{i=1}^{16} W_{2}(i) \cdot \frac{3}{0.1+10^{-\left[\bar{R}(i)+W_{1}(i) / 10\right]}},
$$

where $\bar{R}(i)$ is the average of $R(i)$ over all the frames and $W_{1}(i)$ and $W_{2}(i)$ are the ap" propriate weighting functions accounting for the hearing threshold and the perceptual importance of the $i$-th frequency band, respectively.

\subsubsection{Bark Spectral Distortion}

In [21], Schroeder et al. have described a method of calculating an objective measure for signal degradation based on the measurable properties of the auditory perception. Motivated by this work, a series of psychophysical experimental curves has been invoked in [37] to define a Bark spectral distortion (BSD) measure. At first, a nonlinear frequency transformation from Hertz $f$ to Bark $b$ is made via the relation [21]

$$
f=600 \sinh (b / 6)
$$

which transforms the original power spectral density function $X(S)$ to a critical band density function $Y(b)$. The function $Y(b)$ is 'smeared' by a prototype critical band filter $F(b)$ given as [38]

$$
10 \log _{10} F(b)=7-7.5(b-\alpha)-17.5\left[0.196+(b-\alpha)^{2}\right]^{1 / 2}
$$

with $\alpha=0.215$. The smearing is conceived of as a convolution operation between $F(b)$ and $Y(b)$ which yields a continuous spectrum $D(b)$. The fact that the ear is not equally sensitive to the amount of energy at different frequencies is exploited next. The well-known equal loudness level curves [39] have been used to translate the sound pressure levels (SPL) in $\mathrm{dB}$ to the loudness levels in phons. The increase in phons required to make the subjective loudness double depends on the loudness level and thus finally a phon-to-sone conversion is performed using [38]

$$
S= \begin{cases}2^{(P-40) / 10} & \text { if } P \geq 40 \\ (P / 40)^{2.642} & \text { if } P<40\end{cases}
$$

to generate a Bark spectrum $S(i)$. The BSD measure is defined in [37] as the average of $\mathrm{BSD}^{(k)}$ with

$$
\operatorname{BSD}^{(k)}=\sum_{i=1}^{N}\left[S_{x}^{(k)}(i)-S_{y}^{(k)}(i)\right]^{2}
$$


where $N$ is the number of critical bands; and $S_{x}^{(k)}(i)$ and $S_{y}^{(k)}(i)$ are the Bark spectra in the $i$-th critical band for the $k$-th speech segment corresponding to the original and the coded speech, respectively.

The success of the BSD measure has demonstrated the advantage of considering important perceptual events while formulating a distortion measure. Recently, a software package, named PERCEVAL, is introduced in [40] which computes the probability of detection of the noise as a function of time for noise-corrupted audio and music signals.

\subsection{Summary}

In this chapter, we have reviewed some of the existing subjective and objective measures used in the speech coding area. The mean opinion score and the diagnostic acceptability measure are two of the widely used subjective measures. The most popular class of the time-dnmain measures is the SNR with its variants such as the segmental SNR, the granular segmental SNR etc. Among the spectral distortion measures, the $\log$ likelihood ratio measure, the log area ratio measure, the log spectral distortion measure, the cepstral distance and the Itakura-Saito distortion measure are quite well-known. Some of the existing objective measures have placed emphasis on the aspects which are perceptually important. Two such psychoacoustically-motivated measures are the information index and the Bark spectral distortion measure. The merit of considering important perceptual events has been demonstrated by the success of these measures. 


\section{Chapter 3}

\section{Auditory Representation of Speech}

\subsection{Introduction}

The formulation of any distortion measure requires resolution of two important issues: (i) defining a suitable domain where the signal parameters should be compared and (ii) comparing them in a meaningful sense. This chapter is concerned with the first issue as relevant to speech signals. It has been observed that even the repeated utterances of a sentence by a speaker often differ considerably in the time-domain. In this regard, a spectral representation of speech has appeared to be a relatively steady one. However, we argue that neither the time-domain nor the frequency-domain, in isolation, is a good representation for speech signal. Since a human auditory system is the final information processor in speech communication, it would be meaningful to represent the speech signal in a perceptual-domain (PD). In this work, we use an auditory model for mapping the time-domain speech signal onto its corresponding $\mathrm{PD}$ representation.

The present chapter is organized as follows. Section 3.2 briefly studies the mechanism of the auditory system. Section 3.3 presents various well-established psychoacoustic observations pertinent to speech perception. Section 3.4 discusses four broad classes of analogous electrical model featuring primary auditory processing. In particular, we describe Lyon's auditory (cochlear) model which is used to define the 
PD representation for the present work.

\subsection{Mechanism of Auditory System}

An ear consists of three sections-the outer ear, the middle ear and the inner ear. Speech pressure variations, directed towards the eardrum by the outer ear, are transformed into mechanical motion by the middle ear. Finally, the inner ear converts these mechanical vibrations into electrical firings (impulses) which are sensed by the hair cells and propagated to the brain following an ascending auditory pathway over nerve fibers $[2,41,42]$. In the following subsections, we concisely describe anatomy and functions of the prime components of the auditory system.

\subsubsection{Outer and Middle Ear}

The pinna which is the visible part of the outer ear channelizes sound waves into the ear canal (meatus) and finally hits the eardrum (tympanic membrane). This $2.7 \mathrm{~cm}$ long canal with about $0.7 \mathrm{~cm}$ diameter behaves as a quarter-wavelength resonator and amplifies energy between $3 \mathrm{kHz}$ and $5 \mathrm{kHz}$ by up to $12-15 \mathrm{~dB}$. The middle ear which contains three tiny, dense bones (malleus, incus and stapes) transmit the sound wave vibrations to the oval window membrane of the inner ear. This way, it acts as an acoustic transformer matching the airborne-sound impedance of the outer ear to the fluid-borne sound impedance of the inner ear. The transformer action is due to the ratio of the area of the active parts of the eardrum to the area of the footplate of the stapes. The acoustic impedance of the inner ear fluid is about 4,000 times that of air and this impedance mismatch is such that, without the transformer effect of the ossicles, all but $0.1 \%$ of the pressure waves hitting the eardrum would be reflected back allowing very little energy to enter the inner ear. Additionally, the middle ear also helps in protecting the inner ear against very intense sounds.

\subsubsection{Inner Ear (Cochlea)}

The cochlea, a liquid-filled tube coiled in a snail-shaped spiral, converts mechanical vibrations at its oval window input into electrical excitation on its neural fiber outputs. 
It has a cross-sectional area of about $4 \mathrm{~mm}^{2}$ at its base near the stapes and tapers gradually to about $1 \mathrm{~mm}^{2}$ at its apex. The interior of the cochlea is divided into three chambers-the scala vestibuli, the scala media and the scala tympani. Between the latter two chambers is the basilar membrane (BM) which increases from a width of $0.04 \mathrm{~mm}$ at its base to $0.50 \mathrm{~mm}$ at the apex. The stiffness of the BM varies smoothly over its length. It is stiff and thin at the basal end, but compliant and massive at the apical end (the ratio of stiffness between ends exceeds 100). Therefore, the cochlea near its base is most sensitive to high frequency sounds and as the wave travels down the cochlea, lower and lower frequencies are sensed. The prime feature of the cochlea is that energy in the acoustic wave is separated by frequercy and each place in the cochlea responds best to one frequency, termed as its characteristic frequency. This way, it maps the spectral components of the signal onto the place domain and maintains a tonotopic organization.

\subsubsection{Inner and Outer Hair Cells}

On the top of the BM (within the organ of Corti), there are about 30,000 sensory hair cells arranged in several rows along the length of the cochlea. The endings of the auditory nerve terminate on these hair cells and each of them has about 40-140 hairs. The tips of the outer hair cells, placed in three or four rows, are embedded in the tectorial membrane. These cells usually do not send any information about the sound to the brain. Rather, they function as part of an active amplifier and signallevel controller. On the other hand, the single row of 3,500 inner hair cells that runs along the length of the BM is the primary source of the nerve pulses that travel to the cochlear nucleus and on up to the brain.

\subsubsection{Neural Pathways}

The chemical stimulation of the nerve endings attached to the hair cells produces an all-or-none electrical firings. The auditory firings pass via the cochlear nerve to the ventral and dorsal cochlear nuclei in the medulla. Subsequently, they traverse through the superior olivary complex, the lateral leminscus, the inferior colliculus and finally the medial geniculate body before entering the brain corlex. The stimuli received at the two ears may interart both at the medulla and mid-brain levels. The 
exact neuro-electrical representation of sound stimuli at these various levels is not sufficiently understood.

\subsection{Psychoacoustic Observations}

Auditory system has been studied from different viewpoints by researchers in the field of psychoacoustics, physiology of hearing and speech processing [2, 42, 43]. We note here some of the psychoacoustic phenomena believed to be important in the prrceptual event. This description, although supplementary to [44], is quite selfcontained.

Observation 1 (Ear Canal as an Organ Pipe): The ear canal, about $l=2.7$ $\mathrm{cm}$ long, is an air-filled cavity open at one end (at the pinna) and closed at the other (at the eardrum) [41]. To a rough approximation, the ear canal can be considered as a uniform pipe and it has normal modes of vibration which occur at frequencies where the pipe length is an odd multiple of a quarter wavelength. The first resonance therefore occurs at the frequency $f_{\text {res }}$ given by

$$
f_{\text {res }}=\frac{v_{\text {sound }}}{4 l} \approx \frac{330 \mathrm{~m} / \mathrm{s}}{4 \times 0.027 \mathrm{~m}} \approx 3,000 \mathrm{~Hz}
$$

which aids the ear's sensitivity in this frequency range.

Observation 2 (Impedance Transformation in Middle Ear): The lever action of the ossicles provides a force amplification $(G)$ of about 1.3 [45]. Moreover, the vibrating area of the eardrum ( $A_{\text {eardrum }}$ ) is approximately $55 \mathrm{~mm}^{2}$, compared to the stapes area $\left(A_{\mathrm{stapes}}\right)$ of $3.2 \mathrm{~mm}^{2}$. Therefore, the ratio $(F)$ of pressure applied at the oval window to that applied at the eardrum is given by

$$
F=\frac{G A_{\text {eardrum }}}{A_{\text {stapes }}}=1.3 \times \frac{55}{3.2} \approx 22 .
$$

This impedance transformation (throngh pressure transformation) leads to an increase of about $20 \log _{10} 22 \mathrm{~dB} \approx 27 \mathrm{~dB}$ in sound pressure level (SPL) [Note: $0 \mathrm{~dB} \mathrm{SPL}=$ $10^{-16} \mathrm{~W} / \mathrm{cm}^{2}$ ] within the middle ear [2]. When low-frequency sounds of more than $85-$ $90 \mathrm{~dB}$ SPL reach the eardrum, the middle ear provides some automatic gain control effect via stapedial reflex [46].

Observation 3 (Motion of BM in Cochlea): The motion of the BM in cochlea is quite complicated; however, its total volume displacement at any instant of time 
is equal to the volume displacement of the stapes or of the round window membrane [46]. The velocity of sound ( $v_{\text {coch }}$ ) in cochlear fluid is $1,600 \mathrm{~m} / \mathrm{s}$ and the length of the cochlea $\left(L_{\text {coch }}\right)$ is around $35 \mathrm{~mm}$ [2]. The corresponding base-to-apex time-delay $\left(\tau_{\text {coch }}\right)$ of the sound is given by

$$
\tau_{\text {coch }}=\frac{L_{\text {coch }}}{v_{\text {coch }}}=\frac{0.035 \mathrm{~m}}{1,600 \mathrm{~m} / \mathrm{s}} \approx 20 \mu \mathrm{s}
$$

which indicates that there is essentially no phase delay in pressure along the BM. The mechanical properties (mass, stiffness, is) of the cochlea change very slowly with place. As a consequence, no significant amount of wave energy is reflected back [41].

Observation 4 (Resonances in $B M$ ): The fluid current due to the motion of the BM tends to go through the point of least resistance where the BM compliance reactance annuls its mass reactance [47]. The BM appears to have a 'hole' in that point-to its left, the BM is very stiff (large capacitive reactance) and to its right, the $\mathrm{BM}$ is massive (large inductive reactance). Thus, each place along the $\mathrm{BM}$ resonates most strongly with a pressure wave of a characteristic frequency (CF) associated with it. The frequency response curves corresponding to different places, found by Nobel laureate von Békésy [45], were rather broad and later Mössbaur's gamma-ray-based experiment suggests much sharper frequency response curves [43]. It has also been observed that all the response curves have almost constant Q-factor, thereby implying a fixed ratio of center frequency to bandwidth for all the band-pass filters. Frequency resolution along the BM is best at low frequencies (apical end) whereas the time resolution is best at higher frequencies (basal end). This is primarily due to the fact that a hair cell attached to a high-CF location on the BM fires in response to a broader set of frequencies than does a low-CF hair cell [44].

Observation 5 (Inner Hair Cells as Rectifiers): Fine hairs, called stereocilia, protrude from the ends of the ininer hair cells. They detect the shearing motion of the membranes and act as transducers converting this deflection to an ion current. When the cilia are bent one way, the hair cells stimulate the primary auditory neurons to fire. When the cilia are bent the other way, no pulses are generated. Thus, the inner hair cells act as half-wave rectifiers for the velocity of the motion of the fluid [41].

Observation 6 (Outer Hair Cells as Coupled Gain-Controllers): Studies on the cochlear echo and the oto-acoustic emission suggest that the BM behaves as an active system and the transfer characteristics of the BM system vary depending on the input signal level [48]. This is attributed to the fact that the outer hair cells interact with 
the BM motion. Sounds with high SPLs are effectively diminished whereas sounds with low SPLs are enhanced by the 'superregenerative active' mechanisms of the outer hair cells [46].

An important aspect of hearing is the phenomenon of auditory masking in which the perception of low-energy sound is obscured by the presence of a high-energy sound $[49,50]$. The outputs of the band-pass filters may be viewed as zero-mean 'carrier' signals which are 'amplitude-demodulated' by the half-wave detection nonlinearity. The phenomenon of auditory masking can thus be justified by the 'threshold effect' phenomenon [51] as observed in the envelope detection process of AM signals.

Effects of the outer hair cells can be emulated by automatic gain control (AGC) stages and some kind of inter-stage coupling of these AGCs can simulate the auditory masking feature. Any gain control effect (i.e., amplification or compression) is not instantaneous and the time required to adapt to any input signal is dependent on the signal level [44].

\subsection{Perceptual-Domain Representation}

We desire to deal with an accurate description of human perception as far as possible. But at the same time, since the computational speed of the model is also of importance, we prefer using a functional model of the auditory system for the PD representation of speech signal.

\subsubsection{Auditory Models for Speech Representation}

The interpretation of the cochlea as a spectrum analyzer goes back to Helmholtz [52] in the last century. The timing or volley theory states that low sound frequencies such as those corresponding to the fundamental frequency (F0) of speech, are perceived in terms of time-synchronous neural firings from the BM apex. On the other hand, the place theory suggests that, especially for higher frequencies such as those in the formants of speech, the spectral information is decoded via the BM locations of the neurons that fire most [53].

Current models for representing speech in the auditory periphery falls into 


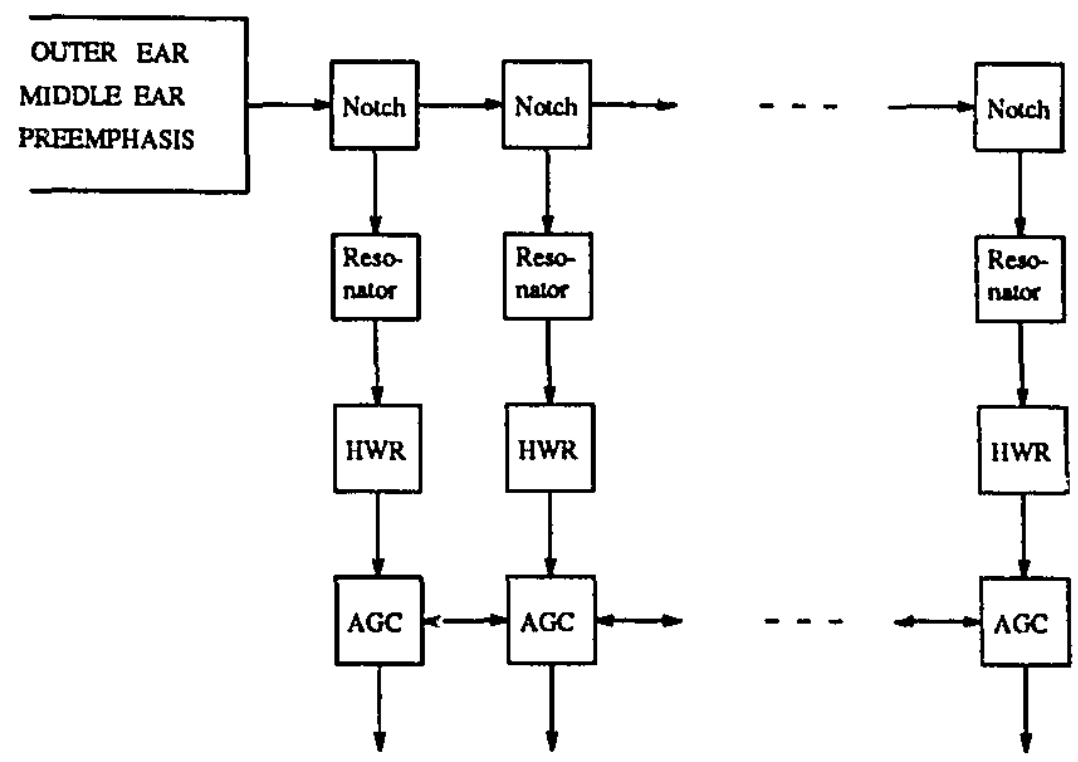

Figure 3.1: Block diagram of Lyon's cochlear model ('HWR' stands for the half-wave rectifier and 'AGC' stands for the automatic gain controller)

one of four broad classes [54]: rate/place, synchrony/place, synchrony/quasi-place and synchrony/place-independent. The rate/place representation [55], $\approx$ well-defined average-rate-based spatial profile, functions well at low SPL. The synchrony/place representational form [56] is based on neural synchrony and requires the system to possess some knowledge of the tonotopic affiliation of each fiber with which to evaluate its temporal firing pattern. The synchrony/quasi-place model [57], in the form of lateral inhibitory network, considers simultaneous activity across adjacent channels. A proposition that a spectral representation based on synchrony need not be concerned with the tonotopic identity of the auditory nerve fibers gives rise to the synchrony/place-independent model [58] which works well only for high SPL.

Based on the psychoacoustic observations discussed in the previous section, we believe that a synchrony/quasi-place model $[57,59]$ is most appropriate for our work as it could operate satisfactorily for high, medium or even low signal levels. Consequently, we adopt one such synchrony/quasi-place model as suggested by Lyon [44] based on work described elsewhere such as [60]. 


\subsubsection{Mapping Using Lyon's Cochlear Model}

Time-domain speech representation is mapped onto a perceptual-domain where the time-place components become the fundamental bases of analysis. The conversion is achieved here using Lyon's cochlear model as described in [44,61]. This model separates complex mixtures of sounds mainly by segregating different frequencies into different places, but also by preserving enough time resolution to separate the responses to different pitch pulses. Therefore, the voiced speech sounds that differ simultaneously in some formants as well as in pitch are separated into recognizably distinct patterns of activity at the output. By a detailed separation of sounds along the time and frequency dimensions, this model paves way for a robust speech analysis technique. Lyon's cochlear model, as shown in Fig. 3.1, integrates the prime features of the 'place' as well as the 'volley' theory. In the following, we describe the model in six steps.

Step 1 (Outer-and-Middle Ear Filter): The outer-and-middle ear effectively adds a slight high-pass response to the system. Assuming that the input speech signals are sampled at a frequency $f_{s}$ of $8,000 \mathrm{~Hz}$, a simple first-order high-pass discrete-time filter with a corner frequency of $300 \mathrm{~Hz}$ is designed to model roughly the effects of the outer and the middle ear. The frequency response of this filter $H_{\mathrm{OM}}(z)$, plotted in Fig. 3.2, is given by

$$
H_{\mathrm{OM}}(z)=\frac{\left(1-\exp \left[-2 \pi \frac{300}{8000}\right] z\right)}{\left(1-\exp \left[-2 \pi \frac{300}{8000}\right] z\right)_{z=1}}=4.76375(1-0.79008 z) .
$$

This filter has unity gain at DC (i.e., at $z=1$ ). For simplification, the AGC mechanism of the middle ear via stapedial reflex is not modeled here [62].

Step 2 (Notch Filters and Resonators): The cochlea is best described by a continuous differential equation [63]; however, it can be modeled by an ensemble of discrete stages in cascade. Lyon, in his proposed cochlear model, uses such discreteplace approximation. An implementation of the discrete-place stages involves combining a series of notch filters that model the traveling pressure waves with a series of resonators that model the conversion of pressure waves into $\mathrm{BM}$ motion $[44,61]$. The notch filters operate at successively lower frequencies so that the net effect is to low-pass filter gradually the acoustic energy which are collected by the resonators corresponding to different places. We consider here sixty-four stages (covering up to $4,000 \mathrm{~Hz}$ ) in cascade, each having a different frequency sensitivity representing the 


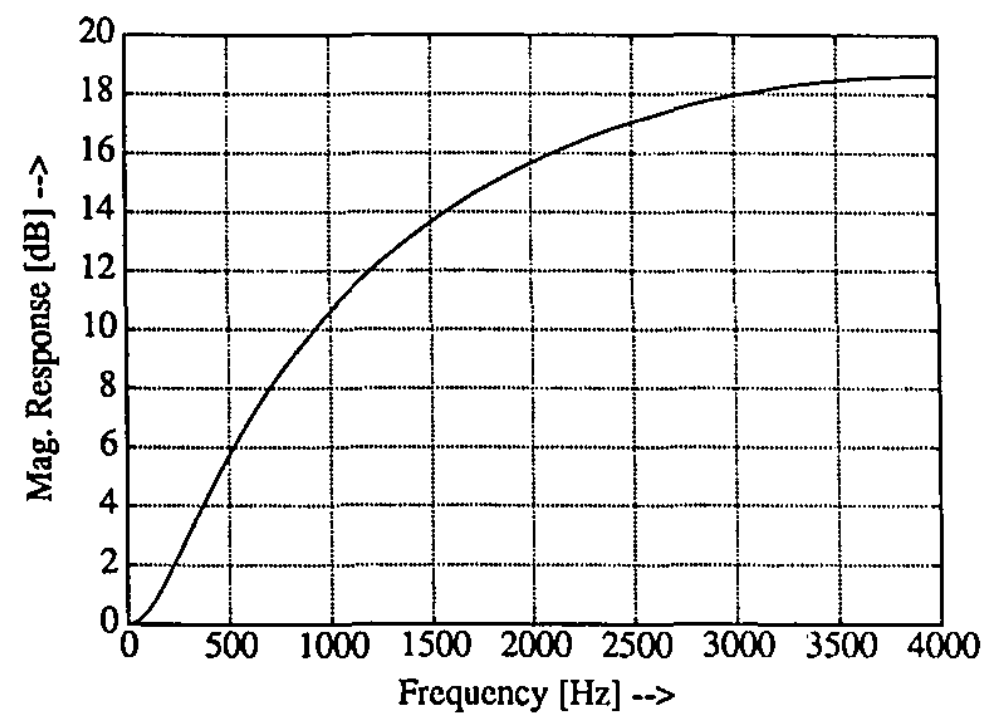

Figure 3.2: First-order outer-and-middle car-filter

associated resonance and is characterized by the respective filter transfer function.

The notch filters and the resonators are approximaled by biquadratic filter transfer functions. Though these stages are designed in discrete-time, Fig. 3.3 plots poles and zeros for some of the notch and the resonator filters, for the sake of clarity, in the $s$-plane. Each of the notch filters has a high-Q zero-pair near a low-Q pole pair whereas each of the resonators has a zero at $D C$ with a high- $Q$ pole pair located between the previous and the next notch filter zero-pairs. Several models of the cochlear mechanics include a micromechanical 'second filter' for a resonance in the organ of Corti that contributes a zero pair slightly below the BM resonance [64]. Presently, this not-so-well-accepted feature is left out. This can easily be incorporated in this model by putting another zero pair in the resonator section.

Step 3 (Cascade Design of Stage Fillers): The combination of the notch filters and the resonators can be implemented in cascade/parallel form as shown in Fig. 3.1. However, to reduce the computations, the notch and the resonator filters of each stage can be integrated into a single ear-filter stage. The locations of the poles in the resonator filters are chosen to be at the same locations as the poles in the succeeding notch filter. This way, the zeros from each notch filter and the poles from a resonator and the next notch filter are integrated to yield a single ear-filter stage [61]. 


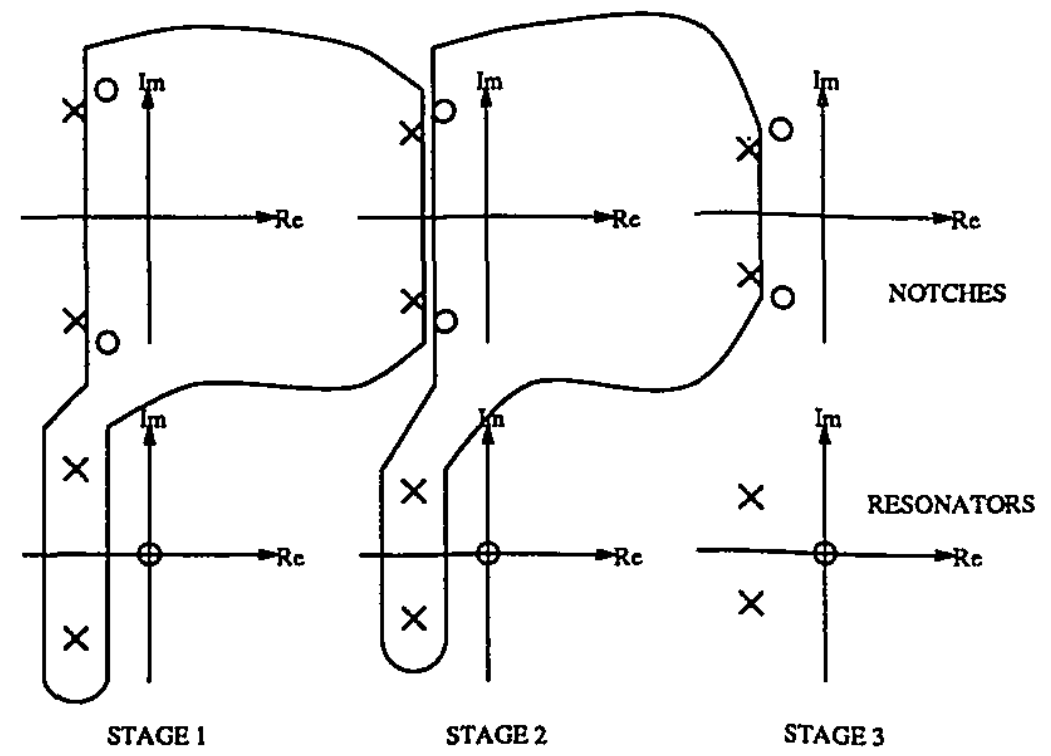

Figure 3.3: $s$-Domain pole-zero plots for typical stages (integrated notch and resonator filters)

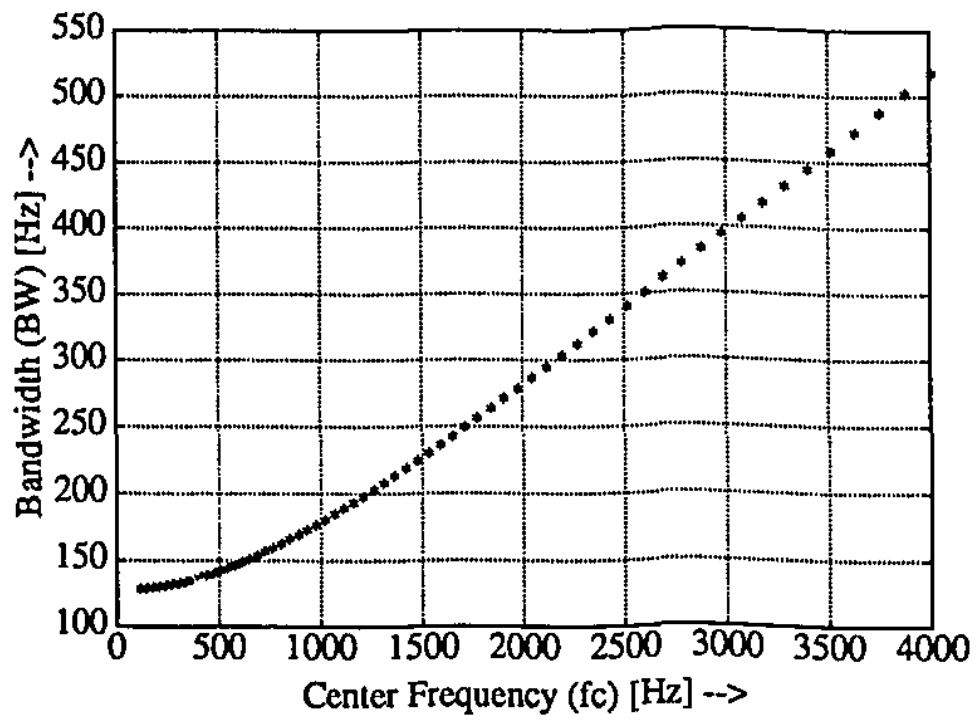

Figure 3.4: Bandwidths vs. center frequencies of sixty-four stages 
The composite transfer function of each ear-filter stage is an asymmetric bandpass function. $W_{\text {ear }}\left(f_{c}\right)$, the 3-dB bandwidth of a band-pass filter with center frequency $f_{c}$, is defined as

$$
W_{\text {ear }}\left(f_{c}\right)=\frac{\sqrt{f_{\mathrm{c}}^{2}+f_{\mathrm{eb}}^{2}}}{Q_{\mathrm{ear}}},
$$

where the ear-break frequency $f_{\mathrm{eb}}$ is $1,000 \mathrm{~Hz}$ and the constant Q-factor for ail the band-pass filters $Q_{\text {ear }}$ is 8 . In conformance to psychoacoustical data, four successive ear-filter stages are overlapped within the $3-\mathrm{dB}$ bandwidth of any one ear-filter and thus we have, $S_{\text {ear }}$, reciprocal of the number of overlapping ear-filter stages, as 0.25 . Finally, the following parameters are obtained for any ear-filter stage corresponding to a particular characteristic frequency:

$$
\begin{array}{r}
f_{c p}=f_{c} ; \quad Q_{c p}=\frac{f_{c p}}{W_{\mathrm{ear}}\left(J_{c}\right)} \\
f_{c z}=f_{c}+W_{\mathrm{ear}}\left(f_{c}\right) \cdot S_{\mathrm{ear}} \cdot Z_{\mathrm{off}} ; \quad Q_{c z}=h_{\mathrm{ear} \cdot} \cdot \frac{f_{c z}}{W_{\mathrm{ear}}\left(f_{c}\right)},
\end{array}
$$

where $f_{c p}$ and $f_{c z}$ are the center frequencies of the associated poles and zeros of a particular ear-filter stage having center frequency $f_{c}$. The center frequency of the associated zero is an extra stage higher than that of the pole. Thus, the $Z_{\mathrm{off}}$, a factor that determines how far the zero is offset from the center frequency of the ear-filter stage, is chosen to be 1.5. $Q_{c p}$ and $Q_{c z}$ are the $Q$-factors for the corresponding poles and zeros and the parameter $h_{\mathrm{ear}}$, which determines how much sharper the notch (zero) is than the resonator (pole), is selected to be 5.0 .

The ear-filter stages are indexed from 1 (corresponding to the highest frequency) to 64 (corresponding to the lowest frequency) and the center frequency of each stage decreases by $S_{\text {ear }}$ (here, 0.25 ) times the bandwidth of the previous stage. $W_{\text {ear }}\left(f_{c}\right)$ vs. $f_{c}$ of all the sixty-four ear-filter stages are plotted in Fig. 3.4 where we observe that $\lim _{f_{c} \rightarrow 0} W_{\text {ear }}\left(f_{c}\right) \rightarrow \frac{f_{\text {eb }}}{Q_{\text {ear }}}=125$.

Step 4 (Other Adjustments in Stage Filters): To implement the zeros at DC for every resonator, a differentiator is required for each stage. Since all the filtering used is linear, the differentiator ( $a$ term of the form $1-z$ ) can be placed just once before the ear cascade. In addition, the differentiator is combined with a zero at the Nyquist rate $(1+z)$ to compensate for the close spacing of the poles near $z=-1$ for high frequency. The frequency response for this combined filter is given as

$$
H_{\text {comb }}(z)=0.5\left(1-z^{2}\right)
$$




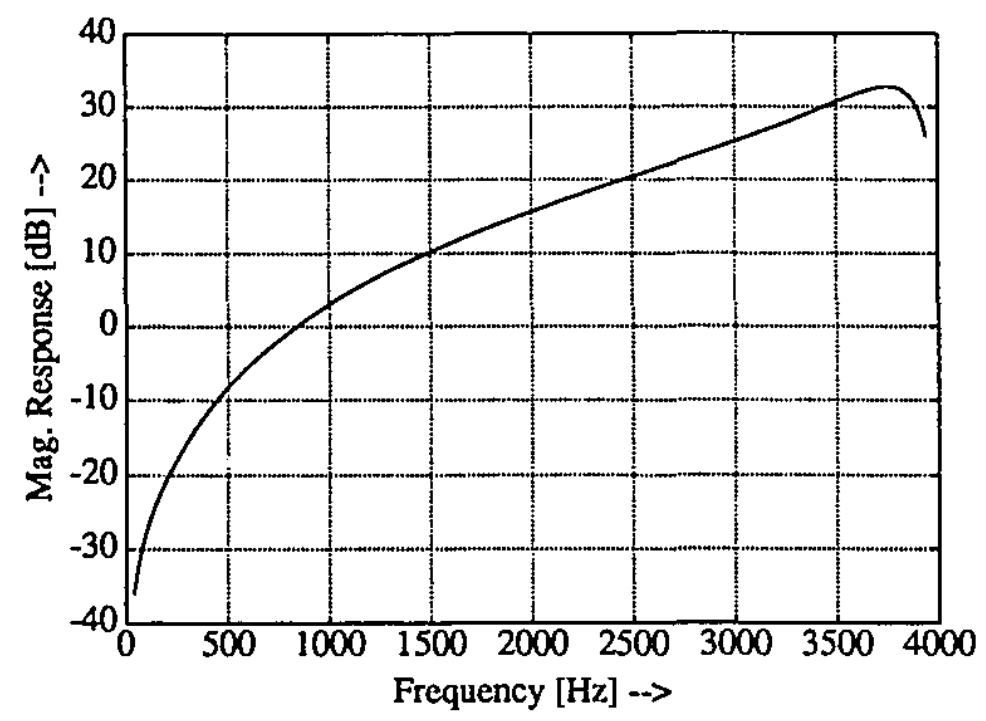

Figure 3.5: Initial stage filter

with unity gain at one-quarter of the sampling frequency.

In the cascade form, each of the ear-filter stages is implemented by a combination of two poles and two zeros. After the pole-zero integration, a pair of poles of the first stage is left aside. Thus, the ear-filter is redefined with an initial stage $H(z)$ which combines the effects of the outer-and-middle ear $H_{\mathrm{OM}}(z)$ and the differentiatorcompensator $H_{\text {comb }}(z)$ with the two poles of the first stage filter. The transfer function of this initial stage filter becomes

$$
H(z)=\frac{(-0.77356+3.91442 j)(1-0.79008 z)\left(1-z^{2}\right)}{0.67523+1.64342 z+z^{2}}
$$

and the corresponding magnitude frequency response plot is shown in Fig. 3.5.

The gain of an ideal differentiator is proportional to frequency. Preceding all stages of the ear-filter with a single differentiator causes the lower frequency stages to have a much lower output than the preceding stages. While within a single stage, it is desired to add a term that is proportional to frequency, the effect of differentiator at each stage is adjusted so that it has unity gain at the center frequency of the corresponding stage. Typical frequency responses for three ear-filter stages with center frequencies as $499 \mathrm{~Hz}, 1,013 \mathrm{~Hz}$ and $2,509 \mathrm{~Hz}$ are shown in Fig. 3.6.

Step 5 (Half-wave Rectification): The exact shape of the half-wave nonlinearity 


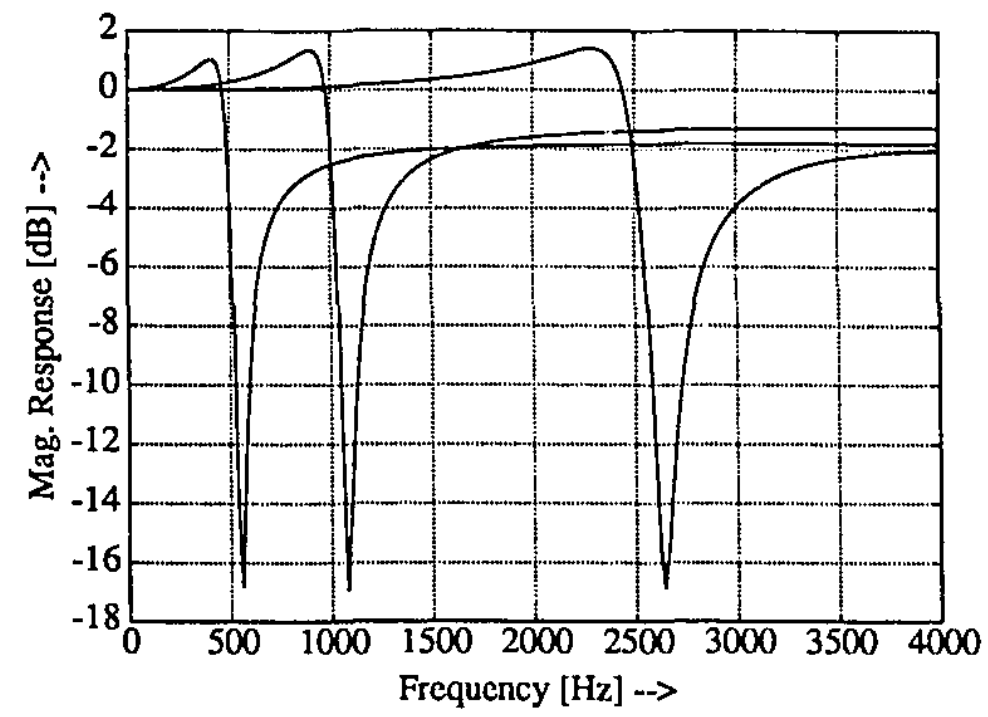

Figure 3.6: Magnitude responses for three typical ear-filter stages with $f_{c}=499 ; 1,013$ and $2,509 \mathrm{~Hz}$

is not obvious; there are proposals for ideal as well as soft half-wave [65] rectification. In this work, an ideal half-wave rectifier is considered.

Step 6 (Coupled Automatic Gain Controllers): The effects of the BM and the hair cell nonlinearity are taken care of adequately by lumping them into a gain control mechanism. Other nonlinear effects, such as the cubic difference tones etc., are assumed to be relatively unimportant to normal hearing [41].

The most important adaptation mechanism in sensory systems is lateral inhibition by which the sensory neurons reduce their own gain as well as the gain of the others nearby. A logarithmic or simple non-coupled AGC mechanism does not adequately handle wide variations of energy across the frequency dimensions. Therefore, Lyon proposed a coupled AGC that adapts in the frequency domain [44]. One such coupled AGC, as described in [61], is shown in Fig. 3.7. Each stage is coupled directly only to its neighboring stages. However, in principle, any stage can affect all the other stages having an effect, perhaps, decaying exponentially with distance from it [66]. The gain offered to an input in an AGC stage varies betwosin 0 and 1, and this gain factor is determined based on the previous states of the current, the left and the right stages as well as the previous output value. 


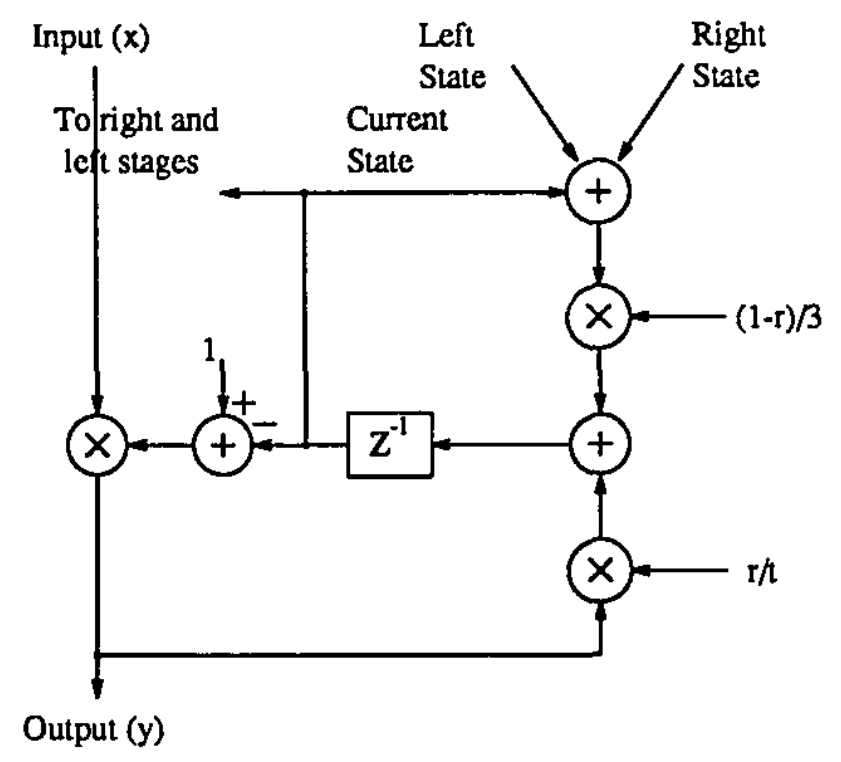

Figure 3.7: A typical automatic gain control (AGC) stage

The time constant of the coupled AGC is made dependent on the signal level. A cascade of four AGC blocks with different time constants, simulating the different adaptation times in the ear, are used [61]. A longer time constant implies that the AGC takes longer to respond to the input. Each AGC attenuates the incoming signal so that, under steady-state condition, it remains below the target value corresponding to that AGC. The target parameters $(t)$ and the time constants $(\tau)$ of the four AGC blocks, respectively, are chosen as $0.0032,0.0016,0.0008$ and 0.0004 units (on the same scale, the amplitude of a signal with $+120 \mathrm{~dB}$ SPL is assumed to be unity) and $640 \mathrm{~ms}, 160 \mathrm{~ms}, 40 \mathrm{~ms}$ and $10 \mathrm{~ms}$. The $r$ parameters as indicated in Fig. 3.7 are related to the $\tau$ parameters as

$$
r=\exp \left[-\frac{1}{\tau f_{s}}\right]
$$

For any one of the sixty-four stages, a typical steady-state response of the four cascaded AGC blocks is depicted in Fig. 3.8.

\subsubsection{Auditory Representation}

The outputs of the cochlear model vary over only about two orders of magnitude as the input signal varies over the entire range covering the threshold of hearing 


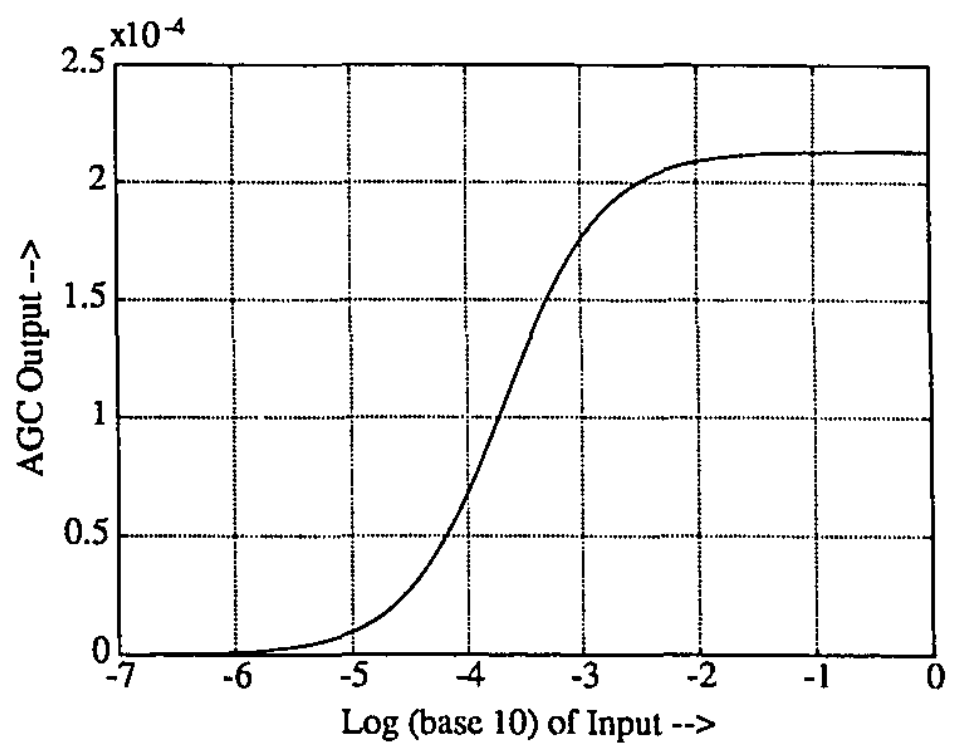

Figure 3.8: A typical steady-state response of four cascaded AGC blocks

to the threshold of pain. The neurons are attached to the hair cells at different places along the cochlear partition and they 'fire' (i.e., generate all-or-none clectrical spikes) based on the gain-controlled signals as sensed by the corresponding hair cells. Essentially, these neural firing events are communicated from the auditory system to the brain through a large number of neural fibers. These neural pathways are termed hereafter as the 'neural channels' so as to keep conformity with the other communication channels. Although these neural fibers are spread densely along the $\mathrm{BM}$, since we consider sixty-four discrete-place stages, we would visualize that all the neurons could be classified into sixty-four characteristic neural channels.

The normalized cochlear model output provides the probability-of-firing information in these sixty-four neural channels at each clock time. Here, the normalization is done with respect to the maximum possible outpul value (i.e., 0.000213 unit as shown in Fig. 3.8) of the four cascaded AGC blocks and the clock time is chosen to be same as the sampling time, i.e., $125 \mu \mathrm{s}$. Since we do not know the exact firing process, the neural activity patterns can be presented in a cochleagram matrix form which gives the probability-of-firings in all the neural channels for all the clock times. In our work, this auditory representation is referred to hereafter as the perceptual-domain (PD). We assert that, to devise a distortion measure for speech signals, the original and the coded/distorted signal should be compared in this perceptual (time-place) 
domain, rather than just in the time or in the frequency domain. In the next two chapters, we propose two distinct approaches for comparing these PD parameters. These comparisons, in turn, provide measure values to assess the degree of overall coder distortions in a coded/distorted speech with reference to its original version.

\subsection{Summary}

This chapter of the dissertation has dealt with the issue of auditory representation for speech signal which is the first step in our formulation of distortion measures for coded speech. For comparing an original speech with its coded version, neither the time-domain nor the frequency-domain representation is sufficient. It is important to consider all the major perceptual events and represent the speech signal in a joint time-place domain. Towards this end, we have used Lyon's auditory model. This model has simulated the high-pass behavior of the outer-and-middle ear, the bandpass characteristics of the inner ear (cochlea), the half-wave nature of the inner hair cells and the automatic gain controlling feature of the outer hair cells. Temporal and spectral masking effects have also been emulated by inter-stage coupling. The final perceptual domain representation of the speech signal is in the form of firing probabilities in the neural channels at the clock times. 


\section{Chapter 4}

\section{Cochlear Discrimination Information (CDI) Measure}

\subsection{Introduction}

In Chapter 3, we have addressed the issue of representing speech signal in a perceptualdomain (PD). This PD representation is a sequence of $N$-dimensional (in our work, $N=64$ ) vectors at the clock times within a speech signal. Each of the $N$ neural channels may be conceived as communication channels with an input alphabet of size two, i.e., firing and non-firing. Due to the lack of our knowledge about the exact neural conversion process, we compare the probability distributions for firing and non-firing, derived from an original and a coded signal, to quantify the degree of distortion. The discrimination information which has emerged as a powerful tool [67] for nucasuring the 'closeness' of two probability density or distribution functions is applied herc for defining a cochlear discrimination information (CDI) measure $[68,69]$. In the first part of this chapter, we formulate the CDI measure and study speech coder performances with it.

For any source-coder, a source-destination p.2.ir can be characterized by a probabilistic model of the source and a fidelity criteri $n$ measuring the degradation of the coded signal with reference to the original source. Based on the rale-distortion theory, a rate-distortion function $R(D)$ may be associated with any such source-destination pair. This function calculates the effective rate at which the source produces infor- 
mation subject to the constraint that an average distortion of $D$ is endured at the destination. A knowledge of the $R(D)$ is of considerable importance as it may prevent one from frivoling time as well as resources to achieve an impossible task. However, often, it becomes difficult to give an explicit closed-form or parametric solution to the $R(D)$, even for apparently simple sources and distortion measures. In such cases, a lower bound to the $R(D)$ or an algorithm to compute it proves to be helpful. The second part of this chapter provides a rate-distortion-theoretic analysis for speech coding based on the CDI distortion measure.

The remainder of the chapter is organized as follows. Section 4.2 puts forward the irlea of CDI, a perceptual cross-entropy measure-based fidelity criterion for speech signals. Section 4.3 provides some experimental results with relevant remarks. Section 4.4 defines $R(D)$ mathematically, provides preliminary background and surveys pertinent literature. Section 4.5 addresses the $R(D)$ evaluation problem by characterizing a source-destination pair and computing an $R(D)$ function for speech coding directly using the Blahut algorithm. The performance of different speech coders is analyzed with respect to these limits.

\subsection{Distortion Computation}

The cochlear discrimination information (CDI) measure, in effect, determines the amount of new information (the increase in neural source entropy) associated with the coded signal when the neural source entropy associated with the original speech is known or vice versa.

Let $P$ be a set of probability measures defined on a measure space $\mathcal{S}^{(J)}$ for a discrete information source with an alphabet of size $J$. The Rényi-Shannon entropy $H_{\alpha}(P)$ for such source with $P=\left\{p_{1}, p_{2}, \ldots, p_{J}\right\}$ is given as [70]

$$
H_{\alpha}(P)= \begin{cases}-\sum_{j=1}^{J} p_{j} \log p_{j}, & \alpha=1, \\ \frac{1}{1-\alpha} \log \left(\sum_{j=1}^{J} p_{j}^{\alpha}\right), & \alpha \geq 0, \quad \alpha \neq 1 .\end{cases}
$$

It has been shown in $[70,71]$ that 
1. $H_{\alpha}\left(P^{\prime}\right.$ is a continuous positive decreasing function of $\alpha$ and is also continuous in $P$.

2. $H_{\alpha}(P)$ is always non-negative and $H_{\alpha}(P)=0$ if and only if all of the $p_{j}$ 's except one are equal to zero.

3. $\left.H_{a_{i}} P\right)$ is strictly concave with respect to $P$ for $0<\alpha \leq 1$; i.c., $H_{\alpha}\left(\lambda P^{\prime}+\right.$ $\left.\overline{1-\dot{\lambda}} P^{\prime \prime}\right) \geq \lambda H_{\alpha}\left(P^{\prime}\right)+(1-\lambda) H_{\alpha}\left(P^{\prime \prime}\right) \forall P^{\prime}, P^{\prime \prime}$ and all $\lambda \in(0,1)$.

4. Convexity or concavity of $H_{\alpha}(P)$ with respect to $P$ depends on $J$ for $\alpha>1$.

Now, let us consider one neural channel for a specific clock time. Since there are only two events possible (i.e., firing and non-firing), the measure space can be written as

$$
\mathcal{S}^{(2)} \triangleq\left\{P: P=\left(p_{1}, p_{2}\right) ; \quad p_{1}, p_{2} \geq 0 ; \quad p_{1}+p_{2}=1\right\} .
$$

The Appendix A shows that with $P \in \mathcal{S}^{(2)}, H_{\alpha}(P)$ is strictly concave with respect to $P$ not only for $0<\alpha \leq 1$, but also for $1<\alpha \leq 2$. Thus, here we consider $\alpha$ values in the range $[0,2)$ which ensures a global maximum of $H_{\alpha}(P)$ for $p_{1}=p_{2}=1 / 2$.

In this work, time-domain speech representation $\mathcal{T}$ is mapped onto the $\mathrm{PD} \mathcal{A}$ using Lyon's cochlear model $\mathcal{C}$. Mathematically, this mapping $\mathcal{B}$ can be expressed as $\mathcal{B}: \mathcal{T} \stackrel{\mathcal{C}}{\rightarrow} \mathcal{A}$. The PD representation $\mathcal{A}$ for an original speech signal can be written in a matrix form as

$$
\mathcal{A}=\left[\begin{array}{cccc}
P_{11} & P_{12} & \cdots & P_{1 N} \\
P_{21} & P_{22} & \cdots & P_{2 N} \\
\vdots & \vdots & \vdots & \vdots \\
P_{n 1} & P_{n 2} & \cdots & P_{n N}
\end{array}\right]
$$

with $n$ clock times and $N$ neural channels. An element $P_{k l}$ of the matrix $\mathcal{A}$ implies that $p_{1 k l}$ and $p_{2 k l}=1-p_{1 k l}$ are the firing and the non-firing probabilities for the $k$-th neural channel at the $l$-th clock time corresponding to the original speech signal. Similarly, let $q_{1 k l}$ and $q_{2 k l}=1-q_{1 k l}$ be the firing and the non-firing probabilities for the coded/distorted speech. Accordingly, the directed divergence (a form of the 
discrimination information measure) between $P_{k l}$ and $Q_{k l}$ can be written as [71]

$$
D_{\alpha}\left(P_{k l} ; Q_{k l}\right)= \begin{cases}\sum_{j=1}^{2} p_{j k l} \log \left(\frac{p_{j k l}}{q_{j k l}}\right), & \alpha=1, \\ \frac{1}{(\alpha-1)} \log \left(\sum_{j=1}^{2} \frac{p_{j k l}^{\alpha}}{q_{j k l}^{\alpha-1}}\right), & \alpha \geq 0, \quad \alpha \neq 1 .\end{cases}
$$

This measure is not a metric as it does not satisfy some of the conditions required for it to be a metric-(a) the symmetry condition $\left[D_{\alpha}\left(P_{k l} ; Q_{k l}\right)\right.$ is not the same as $D_{\alpha}\left(Q_{k l} ; P_{k l}\right)$ when $P_{k l}$ and $Q_{k l}$ are different]; and (b) the triangle inequality [the sum of the measures $D_{\alpha}\left(P_{k l} ; Q_{k l}\right)$ and $D_{\alpha}\left(Q_{k l} ; R_{k l}\right)$ may be greater than, equal to or less than $D_{\alpha}\left(P_{k l} ; R_{k l}\right)$ for any three probability distributions $P_{k l}, Q_{k l}$ and $\left.R_{k l}\right]$. However, the satisfaction of the non-negativity condition allows it to be considered as a fidelity criterion (even though it is not a metric). We define the directed divergence measure of order $\alpha$ for $0<\alpha \leq 2$, the range in which $H_{\alpha}(P)$ has been shown to be concave with respect to $P \in \mathcal{S}^{(2)}$.

For simplicity, we assume that the neural firing events in different channels and at different clock times are independent. Thus, the neural sources corresponding to the $N$ neural channels and the $n$ clock times form a product source, i.e.,

$$
\mathcal{S}=\times_{l \in \mathcal{L}} \times_{k \in \mathcal{K}} \mathcal{S}_{k l}^{(2)}
$$

with $\times$ as the cartesian product of the probability spaces, $\mathcal{L} \equiv\{1,2, \cdots, n\}$ and $\mathcal{K} \equiv\{1,2, \cdots, N=64\}$. I'nder this assumption, the probability distribution of the product source is the product of the probability distributions of the individual sources [1] and the directed divergence values are additive, i.e.,

$$
D_{\alpha}(P ; Q)=\sum_{l \in \mathcal{L}} \sum_{k \in \mathcal{K}} D_{\alpha}\left(P_{k l} ; Q_{k l}\right)
$$

The salisfaction of (4.6), along with the non-negativity of the directed divergence for $\alpha \geq 0$, are shown in the Appendix B.

One generalized form for the directed divergence measure is the $f$-divergence [72] based on which the distortion measure can be defined as

$$
D_{\mathrm{gen}}(P ; Q)=\sum_{l \in \mathcal{L}} \sum_{k \in \mathcal{K}} \sum_{j=1}^{2} q_{j k l} f\left(\frac{p_{j k l}}{q_{j k l}}\right)
$$


where $f(\bullet)$ is a convex function. This specializes to the directed divergence with $\alpha=1$ (also known as the Kullback-Leibler divergence) if $f(x)=x \log x$; to the $\lambda^{2}$-divergence [72] if $f(x)=(x-1)^{2}$; to the $K$-directed divergence [73] if $f(x)=x \log \{2 x /(1+x)\}$ and to the variational distance [74] if $f(x)=|x-1|$. It may be noted that there exist relationships among many of these measures (e.g., a lower bound for the KullbackLeibler divergence in terms of the variational distance is given in [75]). In this work, we also use a 'symmetrized' divergence measure $S_{\alpha}(P ; Q)$ defined as

$$
S_{\alpha}(P ; Q)=D_{\alpha}(P ; Q)+D_{\alpha}(Q ; P)
$$

The divergence measures based on the entropies other than the Rényi-Shannon type can also be studied. One such common example is

$$
C_{1.5}(P ; Q)=\sum_{l \in \mathcal{L}} \sum_{k \in \mathcal{K}} \sum_{j=1}^{2}\left(\sqrt{p_{j k l}}-\sqrt{q_{j k l}}\right)^{2}
$$

based on the Havrda-Charvat entropy $E_{1.5}(P)$ given as [74]

$$
E_{1.5}(P)=2\left(1-\sum_{j=1}^{2} p_{j}^{1.5}\right) .
$$

In order to maintain the boundelness of the measure, in general, we impose a condition that the probability of firing or non-firing for the original and the coded signal can not be a complete certainty or uncertainty; and accordingly we associate a $1^{-}$or a $0^{+}$probability, as appropriate.

\subsection{Experimental Results}

Twelve speech utterances, of 1-2 sec durations and spoken by male as well as female, were considered for the test. Digitized versions of these speech sentences (listed in the Appendix C) were stored in audio-files having SNR of $50 \mathrm{~dB}$ approximately. Each of these original utterances were passed through six different code-excited linear prediction (CELP)-type speech coders.

No database containing various types of coded/distorted speech with accompanying MOS ratings was available to us. Also, we did not attempt to develop MOS ratings as it implies substantial cost and considerable time. Obtaining such a subjective scale involves the great difficulty of repeatability and elimination of biases and 
artifacts-especially without well-understood anchors. The quantization distortion unit (QDU), defined as the quantity of distortion subjectively equivalent to that of a single encoding of $64 \mathrm{kbps}$ PCM, has often been used in practice as a distortion measure. Recent tests, however, indicate that the QDU may not be as stable and dependable as once it was thought to be [13]. Considering all these aspects, we decided to administer an informal subjective test against which the objective measure results were judged.

In this subjective test, twelve listeners ranked six different coded versions (two with $8 \mathrm{kbps}$ coders $\mathrm{C} 1, \mathrm{C} 2$ and four with $4.8 \mathrm{kbps}$ coders $\mathrm{C} 3, \mathrm{C} 4, \mathrm{C} 5, \mathrm{C} 6)$ of all the twelve speech utterances. The overall perceptual quality of the coded signals was designated as the basis for the order of their preferences. Subsequently, we carried out an objective evaluation of these coded signals with reference to the original speech signal by considering eight variations of the proposed fidelity criterion. These measures were as follows.

1. The directed divergence with $\alpha=1\left[D_{1}(P ; Q)\right]$,

2. The directed divergence with $\alpha=1.5\left[D_{1.5}(P ; Q)\right]$,

3. The directed divergence with $\alpha=2\left[D_{2}(P ; Q)\right]$,

4. The symmetrized divergence with $\alpha=1\left[S_{1}(P ; Q)\right]$,

5. The variational distance $[V(P ; Q)]$,

6. The $\chi^{2}$-divergence $\left[\chi^{2}(P ; Q)\right]$,

7. The $K$-directed divergence $[K(P ; Q)]$ and

8. The Havrda-Charvat entropy-based $\mathrm{C}_{1.5}$-divergence $\left[C_{1.5}(P ; Q)\right]$.

A comparison of the informal listening test results and the objective measure values leads us to make the following remarks.

\subsubsection{Performance of Objective Measures}

In Fig. 4.1, the time-domain waveforms and the spectrograms of an original and three coded versions of a typical speech sentence, say, "Oak is strong and also gives shade" 

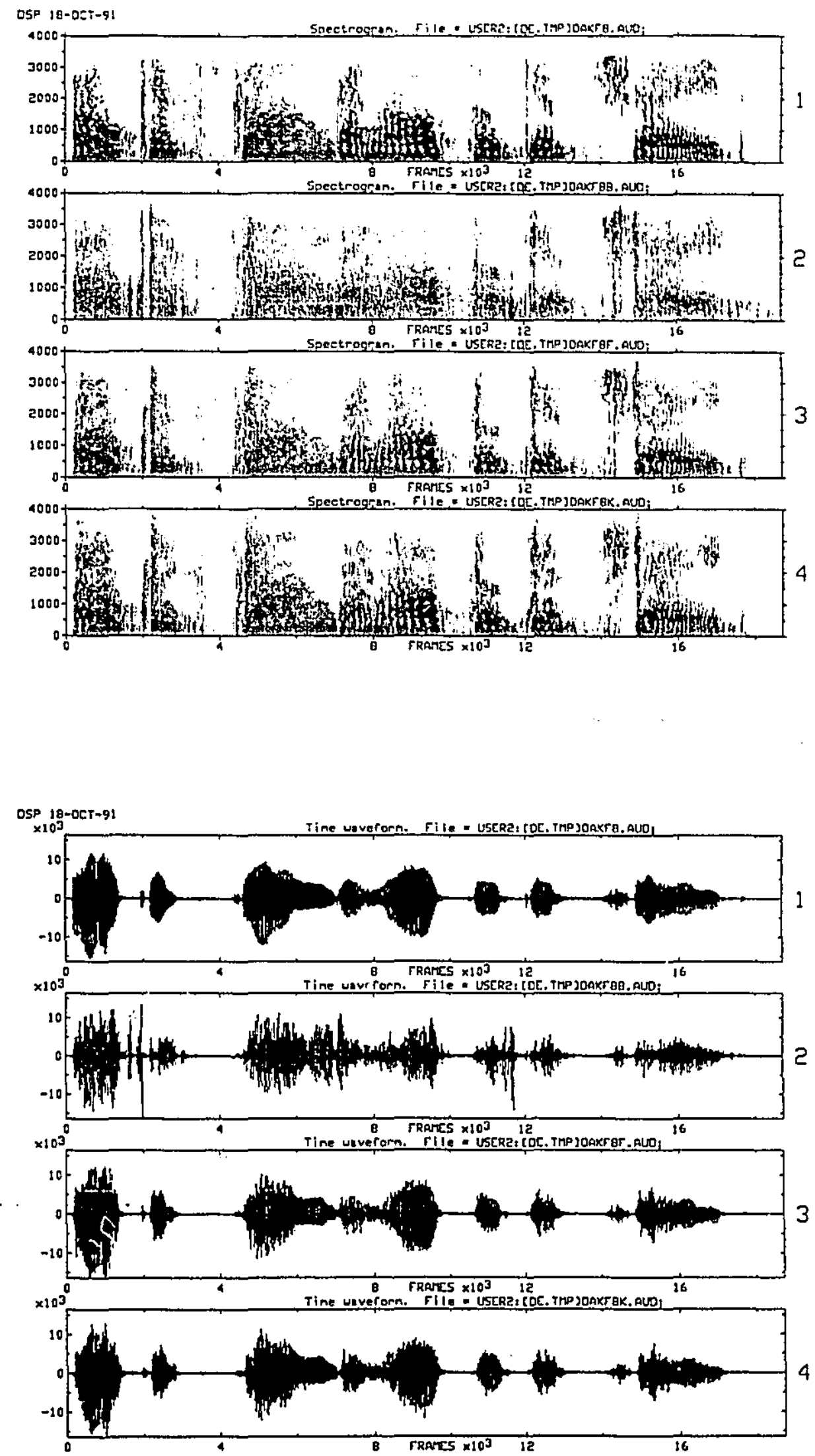

Figure 4.1: Time-domain waveforms and spectrograms of an original and three coded speech signals, "Oak is strong and also gives shade." 
(with 18,800 samples), are shown. Table 4.1 provides average distoition measure values per clock time (with a base-10 logarithm, wherever applicable) for the aforesaid speech utterance. We also tabulate the values of corresponding $\mathrm{SNR}_{\text {seg }}$ as well as SNR with and without scaling ('scaling' implies multiplication of all the coded speech samples by an appropriate factor so as to maximize the SNR value).

In Table 4.2, we provide subjective and objective measure values per clock time for each of the sentences. The subjective rankings ( 6 for the best and 1 for the worst) are averaged over the rankings made by the twelve listeners. These scores are average ordinal numbers and not the absolute quality scores. For each of the twelve utterances and six coded versions, the average ranking scores are mentioned in the first column (marked ' $S$ '). As an example, if a coded signal is given a score of ' 6 ' by eight listeners, a score of ' 5 ' by three listeners and a score of ' 4 ' by one listener, the ' $S$ ' value becomes $(6 \times 8+5 \times 3+4 \times 1) / 12=5.58$.

\begin{tabular}{crrrr}
\hline Measure Type & oakf8f & oakf8k & oakf8b \\
\hline Subjective Ranking & Best & Good & Poor \\
\hline$D_{1}(P ; Q)$ & 2.721 & 2.756 & 4.273 & \\
\hline$D_{1.5}(P ; Q)$ & 4.492 & 4.540 & 6.916 & \\
\hline$D_{2}(P ; Q)$ & 6.751 & 6.812 & 10.165 & \\
\hline$S_{1}(P ; Q)$ & 2.730 & 2.760 & 4.285 & \\
\hline$V(P ; Q)$ & 8.777 & 8.845 & 11.454 & \\
\hline$\chi^{2}(P ; Q)$ & 17.326 & 15.486 & 19.111 & $\times$ \\
\hline$K(P ; Q)$ & 0.795 & 0.806 & 0.909 & \\
\hline$C_{1.5}(P ; Q)$ & 0.077 & 0.083 & 0.117 & \\
\hline SNR (w/o scaling $[\mathrm{dB}])$ & 8.724 & 9.178 & -2.597 & $\times$ \\
\hline SNR (with scaling $[\mathrm{dB}])$ & 8.979 & 9.334 & 0.009 & $\times$ \\
\hline SNR seg $[\mathrm{dB}]$ & 6.815 & 7.080 & -2.004 & $\times$ \\
\hline
\end{tabular}

Table 4.1: Different measure values for three coded signals (with three different $4.8 \mathrm{kbps}$ speech coders) with reference to the original speech utterance F3 (' $x$ ' indicates that the objective measures for 'oakf8f' and 'oakf8k' do not agree with the subjective rankings)

On the other side, we have computed the eight variations of the CDI mea- 
sure values. However, here we tabulate only the $D_{1}(P ; Q)$ measure values (in the second column marked ' $D_{1}^{\prime}$ ') as an example and make general remarks about the other measures. It is emphasized that the lower the amount of additional information (cross-entropy), better is the signal quality of the coded speech with reference to the original one. In Table 4.2, we observe that with the utterance M1, the C4, C5 coders and with the utterance $\mathrm{F} 5$, the $\mathrm{C} 1, \mathrm{C} 2$ coders were ranked same subjectively. Objective measures have shown slight preference towards $\mathrm{C} 4$ coder for $\mathrm{Ml}$ and towards $\mathrm{C} 1$ coder for F5. Besides that, for the utterance F4, the subjective and objective rankings were in contradiction for the coders $\mathrm{C} 1, \mathrm{C} 2$.

\begin{tabular}{ccccccccccccc}
\hline Sent. & \multicolumn{2}{c}{$\mathrm{C} 1$} & \multicolumn{2}{c}{$\mathrm{C} 2$} & \multicolumn{2}{c}{$\mathrm{C} 3$} & \multicolumn{2}{c}{$\mathrm{C} 4$} & \multicolumn{2}{c}{ C5 } & \multicolumn{2}{c}{ C6 } \\
\hline & $S$ & $D_{1}$ & $S$ & $D_{1}$ & $S$ & $D_{1}$ & $S$ & $D_{1}$ & $S$ & $D_{1}$ & $S$ & $D_{1}$ \\
\hline M1 & 5.75 & 2.569 & 4.92 & 2.662 & 4.17 & 2.703 & 2.58 & 2.741 & 2.58 & 2.744 & 1.00 & 4.931 \\
\hline M2 & 5.50 & 2.630 & 5.17 & 2.651 & 4.25 & 2.678 & 2.75 & 2.702 & 2.25 & 2.793 & 1.08 & 4.817 \\
\hline M3 & 5.75 & 2.573 & 5.17 & 2.623 & 4.00 & 2.720 & 2.58 & 2.753 & 2.33 & 2.782 & 1.17 & 4.333 \\
\hline M4 & 5.00 & 2.672 & 5.67 & 2.654 & 4.25 & 2.716 & 2.50 & 2.752 & 2.58 & 2.747 & 1.00 & 4.776 \\
\hline M5 & 5.75 & 2.578 & 5.17 & 2.627 & 3.83 & 2.692 & 2.67 & 2.725 & 2.50 & 2.759 & 1.00 & 4.833 \\
\hline M6 & 5.58 & 2.621 & 5.25 & 2.666 & 3.83 & 2.696 & 2.75 & 2.719 & 2.42 & 2.760 & 1.17 & 4.669 \\
\hline F1 & 5.67 & 2.607 & 5.00 & 2.671 & 4.25 & 2.695 & 2.33 & 2.801 & 2.58 & 2.751 & 1.17 & 4.722 \\
\hline F2 & 5.67 & 2.612 & 5.00 & 2.678 & 3.91 & 2.737 & 2.67 & 2.766 & 2.50 & 2.774 & 1.25 & 4.285 \\
\hline F3 & 5.50 & 2.619 & 5.17 & 2.648 & 4.25 & 2.721 & 2.50 & 2.756 & 2.25 & 2.771 & 1.33 & 4.273 \\
\hline F4 & 5.41 & 2.661 & 5.25 & 2.649 & 4.17 & 2.700 & 2.75 & 2.729 & 2.17 & 2.793 & 1.25 & 4.562 \\
\hline F5 & 5.50 & 2.653 & 5.50 & 2.658 & 3.83 & 2.743 & 2.33 & 2.797 & 2.50 & 2.765 & 1.33 & 4.414 \\
\hline F6 & 5.67 & 2.602 & 4.83 & 2.674 & 4.08 & 2.694 & 3.08 & 2.701 & 2.17 & 2.791 & 1.17 & 4.379 \\
\hline
\end{tabular}

Table 4.2: Subjective and objective measure values for coded signals with reference to the corresponding original speech utterances (M1-M6 (male) and F1-F6 (female) are speech utterances, $\mathrm{C} 1-\mathrm{C} 6$ are speech coders, ' $S$ ' denotes the average subjective ranking scores and ' $D_{1}$ ' gives the directed divergence measure values with $\alpha=1$ )

Over the test sentences, the human rankings were found to be almost consistent with the measures $D_{1}(P ; Q), D_{1.5}(P ; Q), D_{2}(P ; Q)$ and $S_{1}(P ; Q)$; and satisfactorily consistent with the measures $K(P ; Q)$ and $C_{1.5}(P ; Q)$. Furthermore, the $D_{\alpha}(P ; Q)$ class of the measures has shown conformance to subjective evaluation results where 
the SNR measure (with or without scaling) and also the $\mathrm{SNR}_{\text {seg }}$ measure have failed. However, the $V(P ; Q)$ and the $\chi^{2}(P ; Q)$ measures often disagreed with the subjective rankings, especially when two coded signals were very close in their perceptual quality.

\subsubsection{Effect of Different Entropies}

The $D_{1}(P ; Q)$ and the $D_{2}(P ; Q)$ measure profiles for one neural channel at a particular clock time are presented in Fig. 4.2 where the $X$-axis is the probability-of-firing for the original signal, the $Y$-axis is the probability-of-firing for the coded signal in the same channel and the $Z$-axis is the corresponding measure. It was noticed that the value of $\alpha$ in the $D_{\alpha}(P ; Q)$ measure class has a consistent but small effect on its performance. For finer classification (i.e., classifying two coded signals almost equal in their perceptual quality), it has been found to be useful to apply an $\alpha$ value larger than one to increase the dynamic range of the measure values. It has also been observed that the measures based on the Rényi-Shannon entropy show better performance than that based on the Havrda-Charvat entropy.

\subsubsection{Effect of Gain Changes}

The $\chi^{2}(P ; Q)$ and $V(P ; Q)$ measure profiles with the same $X, Y$ and $Z$ axes as of Fig. 4.2 are shown in Fig. 4.3. In addition to the AGC nonlinearity, all the measure profiles (except the $V(P ; Q)$ ) exhibit nonlinearity and the measure values are relatively very small in the neighborhood of the $X=Y$ region. This also makes them insensitive to small gain changes. We speculate that a linear profile of the $V(P ; Q)$ measure is responsible for its poor performance. Due to its broad flatness around the $X=Y$ region, the $\chi^{2}(P ; Q)$ measure shows less sensitivity to gain changes; however, this may be the reason for its unsatisfactory performance in the coder evaluation.

\subsubsection{Effect of Sample Delays}

The CDI measures, in general, were found to be relatively less sensitive (compared to the SNR measure) to a slight time misalignment of the coded signal with respect to the original one or vice versa. For example, let us consider the coded speech signals marked 'oakf8f' and 'oakf8k' of Fig. 4.1. Table 4.3 provides the SNR measure 

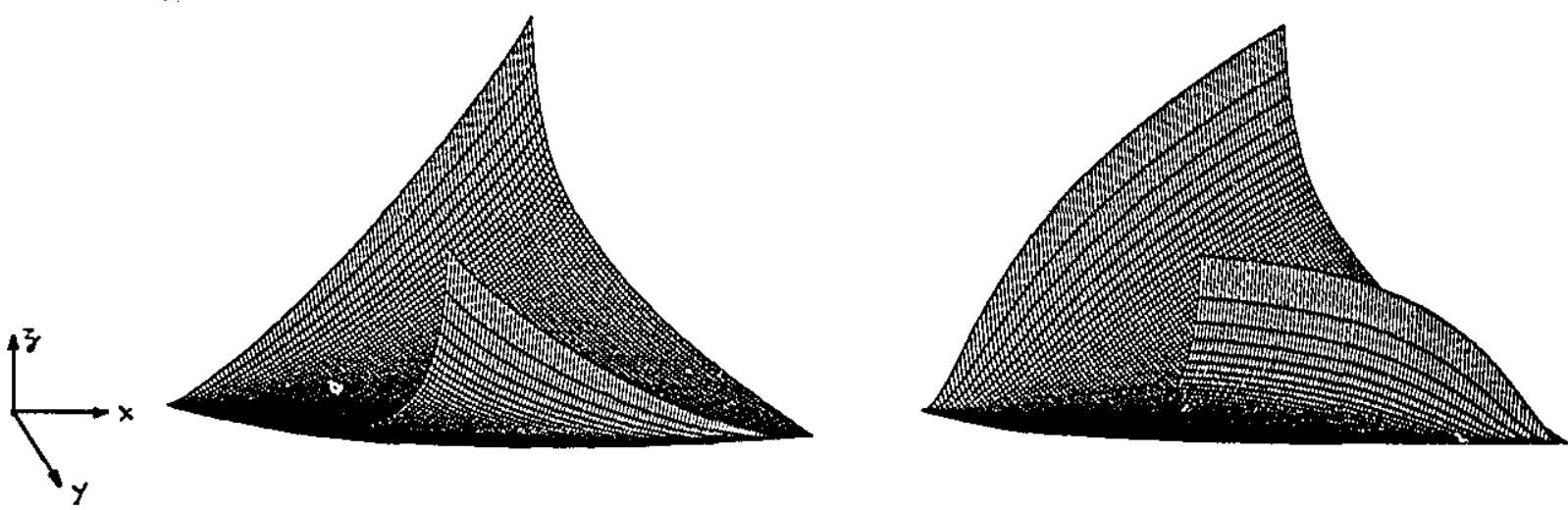

Figure 4.2: The discrimination measure profiles $(J=2)-($ a) the directed divergence with $\alpha=1$ and (b) the directed divergence with $\alpha=2$.

(without scaling) values as well as the $D_{1}(P ; Q)$ and the $D_{2}(P ; Q)$ measure values with zero, one, two and three sample delays in the coded speech. These sample delays are with reference to the original signal and the misaligned sample places are filled in with zero values. In general, we observe that one sample delay does not cause much change in the CDI measure values, but two or three sample delays have considerable effect. With three sample delays, the measures show 'oakf8f' to be inferior to 'oakf8k' (which is aligned to the original signal) although subjectively the reverse is true.

\begin{tabular}{cccccc}
\hline Coded Speech & Measure & \multicolumn{4}{c}{ Sample Delays } \\
\hline & & Zero & One & Two & Three \\
\hline oakf8f & SNR (w/o scaling $[\mathrm{dB}])$ & 8.724 & 7.391 & 5.619 & 5.117 \\
\hline oakf8f & $D_{1}(P ; Q)$ & 2.721 & 2.728 & 2.747 & 2.779 \\
\hline oakf8f & $D_{2}(P ; Q)$ & 6.751 & 6.792 & 7.193 & 8.838 \\
\hline oakf8k & SNR $($ w/o scaling [dB] $)$ & 9.178 & 7.503 & 6.108 & 7.027 \\
\hline oakf8k & $D_{1}(P ; Q)$ & 2.756 & 2.762 & 2.791 & 3.128 \\
\hline oakf8k & $D_{2}(P ; Q)$ & 6.812 & $6.85,5$ & 7.124 & 8.950 \\
\hline
\end{tabular}

Table 4.3: The directed divergence (with $\alpha=1,2$ ) measure values with zero, one, two and three sample delays for the coded signal 'oakf8f' and 'oakf8k' with reference to the original speech sentence 

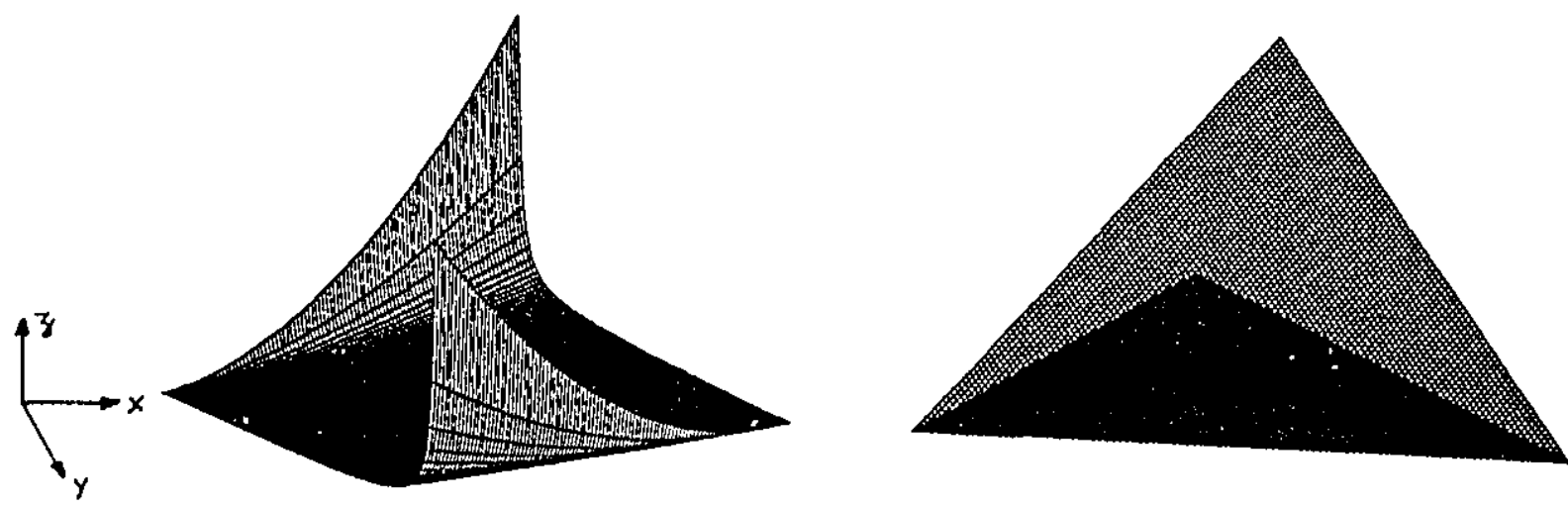

Figure 4.3: The discrimination measure profiles $(J=2)-(\mathrm{a})$ the $\chi^{2}$ divergence and (b) the variational distance.

\subsubsection{Speech Coder Identification}

By considering the neural pathway to be a noisy channel, the subjective evaluation of the speech coders can be treated as a hypothesis testing problem. Csiszár and Longo [76] have shown that the probability-of-error of optimum hypothesis testers based on blocks of measurements decreases exponentially with the block length. Let us consider two coded speech of the same utterance and let $\gamma^{*}$ be the smallest probability that ' $C$ ' is identified to be the samples of ' $A$ ' when it is actually the samples from ' $D$ '. 'This probability is smallest over all the decision rules such that the probability of other type of error (i.e., ' $C$ ' chosen as samples of ' $\mathrm{B}$ ' when it is actually from 'A') does not exceed $\beta$. Then, $\gamma^{*}$, for all $\beta$ in $(0,1)$ and with $\alpha=1$, can be given as [76]

$$
\gamma^{*} \sim \exp \left[-\sum_{l \in \mathcal{L}} \sum_{k \in \mathcal{K}} n^{n}\left(P_{k l} ; Q_{k l}\right)\right]
$$

We conducted an experiment where the listeners were asked to listen to two coded speech sentences ' $A$ ' and ' $B$ ' and then a varying number of samples ' $C$ ' from one of them, not known to the listeners which one, were played. In such subjective evaluation testing, there is no precise way of determining $\gamma^{*}$. The $\gamma^{*}$ could be estimated by carrying out the test with a large number of listeners and then considering their opinions (whether ' $A$ ' or ' $B$ ') about ' $C$ '.

It would be of academic interest to investigate the validity of the relationship of (4.11). In our experiment, we only verified that to achieve a given probability of 


\begin{tabular}{crrrr}
\hline Sentence & Sample Nos. & C3-C4 & C4-C5 & C5-C6 \\
\hline M1 & 3,000 & $5 / 12$ & $4 / 12$ & $7 / 12$ \\
\hline & 6,000 & $7 / 12$ & $7 / 12$ & $9 / 12$ \\
\hline & 9,000 & $11 / 12$ & $10 / 12$ & $12 / 12$ \\
\hline F3 & 12,000 & $12 / 12$ & $12 / 12$ & $12 / 12$ \\
\hline & 3,000 & $6 / 12$ & $4 / 12$ & $8 / 12$ \\
\hline & 6,000 & $8 / 12$ & $6 / 12$ & $11 / 12$ \\
\hline & 9,000 & $11 / 12$ & $9 / 12$ & $12 / 12$ \\
\hline & 12,000 & $12 / 12$ & $11 / 12$ & $12 / 12$ \\
\hline
\end{tabular}

Table 4.4: Speech coder identification for two sentences M1 and F3 (the sample numbers played and the fraction of listeners who have correctly identified the coders are provided in the table)

decision error, it required more samples (i.e., longer durations) of ' $\mathrm{C}$ ' to be played when ' $A$ ' and ' $B$ ' are of 'near equal' quality (as indicated by our measure) compared to that required when ' $A$ ' and ' $B$ ' are of 'substantially different' quality. Table 4.4 shows, for the same example sentence, the subjective identification of coders (i.c, the number of listeners out of twelve listeners correctly identified the coders) and the corresponding number of samples played. We have considered three coder pairs where C4-C5, C3-C4 and $\mathrm{C} 5-\mathrm{C} 6$ were ranked in the descending order from their perceptual quality 'closeness' point of view. For example, let us consider the utterance F3. In Table 4.4, we observe that by playing 6,000 samples, for $\mathrm{C} 4-\mathrm{C} 5$ coder pair, only onehalf of the listeners could identify the coder correctly, the remaining listeners either identified wrongly or could not decide. On the other hand, with the same number of samples played, the correct coders were identified by two-third of the listeners for C3-C4 pair and by almost all the listeners for $\mathrm{C} 5-\mathrm{C} 6$ pair.

\subsection{Rate-Distortion Analysis}

Rate-distortion theory is a branch of information theory that establishes a mathematical foundation to a source encoding problem. For a particular source-destination pair, a rate-distortion function $R(D)$ could be computed which gives the lowest achievable 
rate with an average distortion of $D$ by the defined fidelity criterion. As $D$ increases, $R(D)$ decreases monotonically and usually becomes zero at some finite value of distortion. In the following, we define the $R(D)$ analytically, discuss important results relevant to this work and review some of the pertinent literature.

\subsubsection{Preliminary Background}

We consider a time-discrete source $\left\{X_{t}, P\right\}$ that produces i.i.d. outputs described by a probability density function $p(x)$. The accuracy of reproduction of $x$ by $y$ is measured by a non-negative distortion measure $\rho(x, y)$. An average distortion

$$
d(q)=\iint p(x) q(y \mid x) \rho(x, y) d x d y
$$

and an average mutual information

$$
I(q)=\iint p(x) q(y \mid x) \log \left\{\frac{q(y \mid x)}{q(y)}\right\} d x d y
$$

where

$$
q(y)=\int p(x) q(y \mid x) d x
$$

are assigned to every conditional probability density $q(y \mid x)$. Then, the rate distortion function $R(D)$ of $\left\{X_{t}, P\right\}$ with respect to the fidelity criterion is defined by

$$
R(D)=\inf _{q \in Q_{D}} I(q)
$$

where the set of all $D$-admissible conditional probability assignments is denoted by the symbol

$$
Q_{D}=\{q(y \mid x): d(q)=D\} .
$$

$I(q)$ is a convex downward function of $q$ which implies that any stationary point of $I(q)$ in $Q_{D}$ must yield the absolute minimum, namely the $R(D)$. Since the above formulation is a convex programming problem, generalized Kuhn-Tucker conditions can be determined to identify the conditional probability distribution which attains the infimum in (4.15). The variational problem defining $R(D)$ can be solved using the method of Lagrange multipliers. An application of this method results in the following parametric expressions for $D$ and $R$ [77]:

$$
D=\iint \lambda(x) p(x) q(y) e^{s \rho(x, y)} \rho(x, y) d x d y
$$


and

$$
R=s D+\int p(x) \log \lambda(x) d x
$$

where,

$$
\lambda(x)=\left[\int q(y) e^{s \rho(x, y)} d x\right]^{-1} .
$$

The slope of any $R(D)$ curve at the point $\left(D_{s}, R_{s}\right)$ is represented by the parameter $s$ which is generated parametrically from (4.17), (4.18) and (4.19). If $\Lambda$, be the set of all non-negative functions $\lambda(x)$ satisfying

$$
c(y)=\int \lambda(x) p(x) e^{s \rho(x, y)} d x \leq 1 \quad \text { for all } y
$$

then,

$$
R(D)=\sup _{s \leq 0, \lambda(x) \in \Lambda_{s}}\left[s D+\int p(x) \log \lambda(x) d x\right] .
$$

For each $s \leq 0$, a necessary and sufficient condition for $\lambda(x)$ to attain the supremum in (4.21) is the existence of a probability density $q(y)$ that is related to $\lambda(x)$ by (4.19) and is such that $c(y)=1$ in (4.20) for almost all $y$ for which $q(y)>0$.

\subsubsection{Relevant Literature}

The rate-distortion theory has been developed in the last two decades for discrete as well as continuous sources. For the evaluation of $R(D)$, two broad approaches are generally adopted.

One approach is to derive the Shannon lower bound $R_{L}(D)$ [77] and then to find conditions for the existence of a $D_{c}>0$ s.t. $R(D)=R_{L}(D)$, for all $D \in$ $\left[0, D_{c}\right]$. With difference-type distortion measures $R(D)$ functions have been calculated for Laplacian, Cauchy and Gaussian sources [77]. This idea is generalized and a parametric solution is provided for a weighted mean-square error distortion measure in [78]. For quotient-type distortion measure (i.e, a measure of the functional form $f(x / y))$ and a source with $p(x)=0$ for $x<0$, a logarithmic transformation of the source variables $x$ and $y$ yields $R(D)$ bounds from the results of the difference distortion measures [79]. With balanced distortion measures (i.e., with distortion matrix containing the same set of entries, perhaps permuted, in each column), the $R(D)$ functions are computed in [80] for discrete memoryless source and in [81] for 
finite-alphabet sources with memory. However, it appears to be difficult with this approach to evaluate $R(D)$ with an arbitrary non-balanced fidelity criterion.

The second approach is to evaluate the $R(D)$ function directly. Some simple examples of the finite-alphabet source-destination pair for which the rate-distortion function that can be determined analytically are provided in [77]. Tan and Yao [82] have evaluated $R(D)$ for a Gaussian source and an absolute-magnitude difference criterion by making a suitable choice of the boundary set (i.e., the value of $y$ for which the condition (4.20) is satisfied with equality). This method has also been applied to a large class of i.i.d. sources having probability densities with constrained tail decay [82]. An efficient algorithm for the direct evaluation of the $R(D)$ function for discrete as well as continuous sources has been suggested by Blahut in [83].

Historically, the application of the rate-distortion theory to the speech process has been hindered because of the lack of a widely accepted probabilstic model of the speech process as well as a meaningful distortion measure. The problem is further complicated by the mathematical difficulties in evaluating the rate-distortion function even if a reasonable source-destination pair is defined. A fairly large set of pdf models is suggested in the literature based on the first-order histograms of Nyquist samples of continuous speech waveforms. The gamma pdf based on the long-term statistics [3], the Laplacian pdf based on the medium-term statistics [84] and the Gaussian pdf based on the short-term statistics [85] are among the more proular ones. An evaluation of the first-order $R(D)$ functions based on these prlfs and difference uivitortion measures are available in [86]; and with Itakura-Saito distortion measure in [79].

\subsection{Evaluation of Rate-Distortion Function}

The objective of this section is to provide a rate-distortion-theoretic analysis for speech coders with the CDI measure. We formulate the problem by characterizing the source-destination pair precisely. Then, the $R(D)$ function is computed ...ing the Blahut algorithm. Finally, the performances of different speech coders are studied with respect to these bounds. 


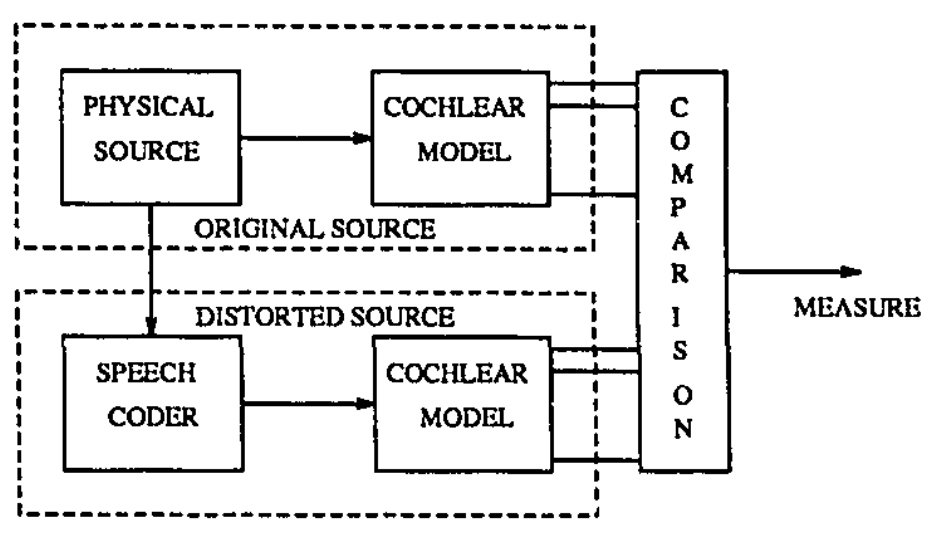

Figure 4.4: Source-destination pair characterization

\subsubsection{Source-Destination Pair Characterization}

The cochlear model is, in essence, a highly non-linear structure with the half-wave rectifiers, the $A G C$ stages and the coupling among them simulating the auditory spectral and temporal masking phenomena. It may prove to be sufficiently difficult to express these signal processing operations, especially the coupling of the AGC stages, with the help of simple mathematical operators. Thus, we take a different outlook towards the source-destination pair model shown in Fig. 4.4. We merge the physical speech source with the cochlear model and consider this ensemble to be tis source. Since there is as such no uniquely accepted pdf for the physical speech source, we are not in any further disadvantageous position by integrating the cuchlear model with the speech source and detcrmining the histogram of the cochlcar model outputs. These outputs, being the probability-of-firing informi, wion, assume values in the range $(0,1)$. The histogram for the firing-probiability is determined by experimenting with twenty-four speech utterances (twelve male and twelve female voices) of 1-2 sec. durations. The firing-probability histogram for each of the sixty-four neural channels could be determined separately. For simplification purpose, we have assumed all the hisiogiams to be identical and derived only one histogram based on the pribabilityof-tiring information obtained from all the chanizels. 


\subsubsection{Calculation Based on Blahut's Algorithm}

In [87], we have derived analytically a lower bound to the $R(D)$ with a single-letter cochlear variational distance measure. However, with the other distortion measure forms, it becomes difficult to give an analytical solution. Moreover, these are not exact solutions; they are merely lower bounds. Here, we use the Blahut algorithm for calculating the $R(D)$ functions exactly.

We treat the probability-of-firing information to be discrete-valued with symbols from one of the 255 uniformly spaced values between 0 and 1 (i.e., $1 / 256,2 / 256, \cdots$, $255 / 256$ ). Let the input alphabet (firing-probability corresponding to the original speech) $u$ be reproduced in terms of an output alphabet (firing-probability corresponding to the coded speech) $v$. Then, the algorithmic steps could be written as follows.

Step 1 : An initial output probability distribution $\left\{Q_{v}\right\}$ is assumed, say, $Q_{v}^{0}$. The parameter set $\left\{A_{u v}=e^{s \rho_{u v}}\right\}$ is evaluated, where $\rho_{u v}$ is the single-letter CDI measure between the input alphabet $u$ and the output alphabet $v$.

Step 2 : The parameter $s$ is chosen from the range of $-\infty$ to 0 ; and then Steps 3 and 4 are caried out with different values of $s$.

Step 3 : With the values of the input probability distribution $P_{u}$ (obtained from the histogram of the cochlear model output) and the parameters $A_{u v}$ the following parameters are calculated:

$$
\begin{aligned}
& c_{v}=\sum_{u} P_{u} \frac{A_{u v}}{\sum_{v} A_{u v} Q_{v}}, \quad Q_{v} \leftarrow Q_{v} c_{v}, \\
& L=\sum_{v} Q_{v} \log c_{v}, \quad U=\max _{v} \log c_{v} .
\end{aligned}
$$

Step 4 : If $U-L \geq \epsilon$, then the previous step is repeated; otherwise, the progiam is terminated for this value of $s$ by evaluating the following:

$$
\begin{gathered}
Q_{v \mid u}=\frac{A_{u v} Q_{v}}{\sum_{v} A_{u v} Q_{v}}, \\
D=\sum_{u} \sum_{v} P_{u} Q_{v \mid u} \rho_{u v}, \\
R(D)=s D-\sum_{u} P_{u} \log \left(\sum_{v} A_{u v} Q_{v}\right)-\sum_{v} Q_{v} \log c_{v}
\end{gathered}
$$




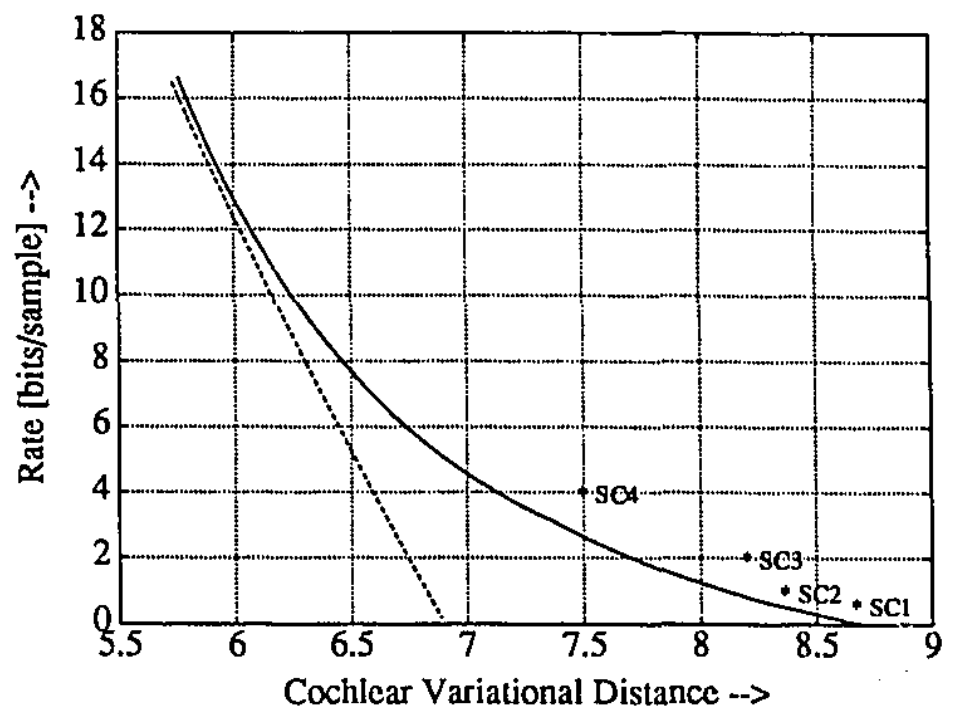

Figure 4.5: Speech coder rate in bits/sample vs. average cochlear variational distance measure ( - - line shows an analytically derived lower bound, _- line shows the exact rate-distortion curve using Blahut's algorithm and four '*' points [SCl-SC4] denote the performances of four speech coders)

Fig. 4.5 shows the $R(D)$ for the $V(P ; Q)$ measure whereas Fig. 4.6 plots the $R(D)$ function for the $D_{1}(P ; Q)$.

\subsubsection{Measured Performances of Speech Coders}

We have considered four state-of-the-art speech coders for the assessment of their average perceptual quality. These four coders (designated as SC1-SC4) were: CELLPbased coder SC1 (4.8 kbps) [5], VSELP-based coder SC2 ( $8 \mathrm{kbps})$ [88], wideband CELP-based coder SC3 (16, kbps) [89] and ADPCM coder SC4 (32 kbps) [3]. For the first, second and the fourth coders with sampling rates of $8,000 \mathrm{~Hz}$, sixly-four neural channels (covering up to $4,000 \mathrm{~Hz}$ band) were assigned as described in this chapter. On the other hand, for the wideband coder with sampling rate of $16,000 \mathrm{~Hz}$, eighty-five neural channels (covering up to $8,000 \mathrm{~Hz}$ band) were assigned as described in Chapter 6. Although we considered only the CELP-type speech coders for comparing the CDI measure performance with subjective assessment, we do not foresee any difficulty in applying this measure to other types of speech coders. With this 


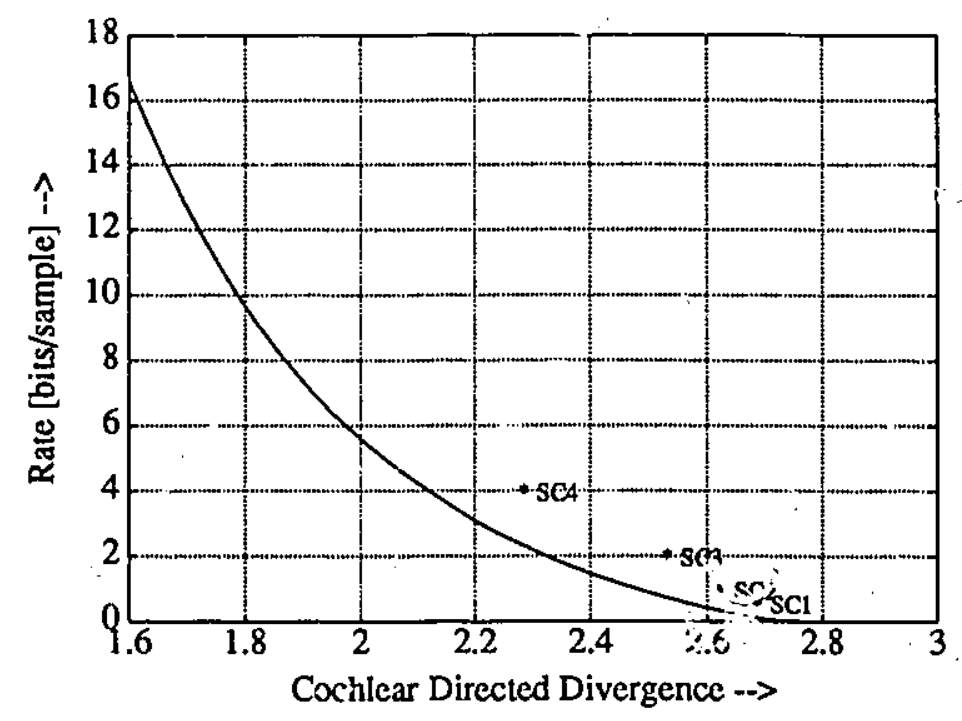

Figure 4.6: Speech coder rate in bits/sample vs. average cochlear directed divergence (with $\alpha=1$ ) measure (— line shows the rate-distortion curve using Blahut's algorithm and four '*' points [SC1-SC4] denote the performances of four speech coders)

understanding, we have included one ADPCM coder in this section to examine its quality with resnect to the rate-distortion limit.

T'welve speech sentences of 1-2 sec dinations were passed through each of the four coders to calculate the average distortion valucs over each sampling time. Fig. 4.5 and Fig. 4.6 plot the performances of the four speech coders (marked by '*') as evaluated by $V(P ; Q)$ and $D_{1}(P ; Q)$, respectively. Now, let us examine one of the figures, Fig. 4.6. We observe that the perceptual quality obtained (measured with the $D_{1}(P ; Q)$ ) by $S \mathrm{~S} 1$ coder is possible to achieve with much lower rate (as low as $1.5 \mathrm{kbps})$. Similarly, SC2, SC3 and SC4 coder performances are achievable with almost $3.8 \mathrm{kbps}, 5.4 \mathrm{kbps}$ and $20 \mathrm{kbps}$, respectively. From another perspective, we can say that a perceptual quality (a value of 2.575 units/sample) somewhere between those attained by SC2 and SC3 coders are attainable with a $4.8 \mathrm{kbps}$ speech coder. A value of 2.485 units/sample which falls between the perceptual quality of SC3 and SC4 is theoretically achievable with an $8 \mathrm{kbps}$ speech coder. Although the rate-distortion analysis does not provide with an answer to how to attain these limits, it gives an insight to what is possible and how close a specific speech coder is performing witli respect to the $R(D)$ limits in terms of the perceptual quality. 


\subsection{Summary}

In this chapter, the firing/non-firing probabilities of original and coded signals were compared in an information-theoretic sense to formulate the cochlear discrimination information measure. This fidelity criterion, in essence, evaluates the ncural firing cross-entropy of the coded speech with respect to that of the original one. The performance of this objective measure was compared with subjective evaluation results. $\mathrm{A}$ low value in this measure has indicated superior quality of the corresponding speech coder. The last part of the chapter has dealt with the calculation of the rate-distortion functions for speech coding based on this distortion measure. For this purpose, we have applied the Blahut algorithm. Four speech coders with rates ranging from 4.8 kbps to $32 \mathrm{kbps}$ were studied from the viewpoint of their performance (as assessed by the cochlear discrimination measure) with respect to the rate-distortion limits. Our study has shown that there is ample scope for the improvement of the coder architecture and the coding algorithm. 


\section{Chapter 5}

\section{Cochlear Hidden Markovian (CHM) Measure}

\subsection{Introduction}

In Chapter 4, we have introduced a cochlear discrimination information measure which exploits the perceptual events at the auditory periphery. In this chapter, we attempt to capture the basics of high-level processing in the brain with simple hidden Markov models. We use these HMMs over the perceptual-domain speech representation and introduce a new measure [90, 91], namely the cochlear hidden Markovian (CHM) measure. Computing coder distortion with the CHM measure involves estimating the HMM parameters from the perceptual-domain observations of an original speech frame and calculating the likelihood (against the estimated HMM) of observing the PD representation corresponding to the coded version of the same speech frame. The proposed CDI measure compares the PD observations directly whereas the CHM measure is a parametric nonlinear model-based measure. Test results, model behavior, advantages/disadvantages of this method and also some other alternatives for measuring coder distortion are discussed.

The format of this chapter is as follows. Section 5.2 characterizes the hidden Markovian signal model. Section 5.3 provides some relevant background materials. Section 5.4 introduces a method to compute distortion for speech coders and also suggests briefly some other alternative approaches. Section 5.5 addresses the HMM 
behavior and tabulates experimental results for speech coder evaluation.

\subsection{Characterization of Hidden Markov Model}

The cochlear model output is a sequence of $K$-dimensional vectors (in our work, $K=64$ corresponding to sixty-four neural channels) with one vector for each clock time $t$. The elements in each of the $K$-dimensional nhservation vectors represent information regarding the probability-of-firing. Based on this PD representation of a speech signal, what are transmitted through neural channels to the brain are series of all-or-none electrical spikes (firings). However, the exact conversion process of the I'D representation to the firing/non-firing representation is not yet known. Wc attempt here to capture the underlying firing/non-firing event in each channel with discrete-time series analysis.

One such analysis technique involves using a hidden Markov model for modeling the observation sequence. The time-varying observation process is considered as a concatenation of many short-time segments of a fixed duration. However, it is expected that the properties of the process change neither synchronously with every analysis duration nor abruptly from each unit to the next one. The development of an efficient optimization technique [92] to estimate the model parameters so as to 'match' the observed signal patterns has culminated in the theory of HMM-based signal representation. $\mathrm{T} \cdot \mathrm{s}$ success of this hidden Markov modeling technique has been proven by its application in ecology (e.g., [93]), text analysis (c.g., [94]), coding theory (e.g., [95]) and speech recognition (e.g., [96]).

An HMM is a doubly embedded stochastic model with an underlying process that is not directly observable (it is hidden), but can be observed through anither set of stochastic processes that produce the sequence of observations. In other words, the states of $a_{1}$ HMM are hidden and the observation is a probabilistic function of the states. The order of occurrence of observations and the correlations among adjacent observations are suitably modeled by stochastic dependencies among the hidden states of an HMM. In the following, we characterize an HMM for our problem by selecting the model type, the number of hidden states and all the parameters associated with the model.

We consider $K$ numbers of independent two-state $(N=2)$ fully-connected mod- 


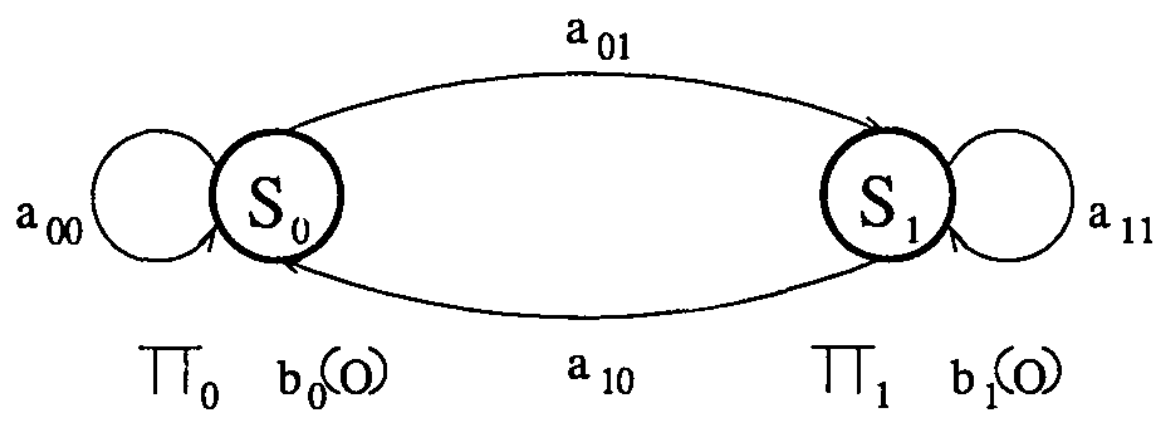

Figure 5.1: A two-state fully-connected hidden Markov model $\left(S_{0}\right.$ and $S_{1}$ denote the non-firing and firing states, $\pi_{0}$ and $\pi_{1}$ are the initial state probabilities, $a_{i j}$ gives the state transition probability from a state $S_{i}$ to a state $S_{j}, b_{0}(O)$ and $b_{1}(O)$ are the observation probability density functions for the state $S_{0}$ and $S_{1}$ respectively)

els, as shown in Fig. 5.1, where either state is reachable from the other one. Although in many applications, the states do not have a physical meaning; here a state $S_{0}$ corresponds to a non-firing event whereas a state $S_{1}$ corresponds to a firing event. The initial state distribution (i.e., at $t=1$ ) is given as $\pi=\left\{\pi_{i} \mid i \in \mathcal{N}\right\}$ with

$$
\pi_{i}=P\left[q_{1}=S_{i}\right] \text { for } i \in \mathcal{N} \text { and } \sum_{i \in \mathcal{N}} \pi_{i}=1
$$

where $\mathcal{N} \equiv\{0,1\}$ and a state reached at any clock time $t$ is denoted by $q_{t}$.

The HMM considered is of order one and hence the transition from one state to the next one occurs according to a transition probability distribution wistch depends only on the previous state. If we define an integer set $\mathcal{T} \equiv\{1,2, \cdots, T-1\}$ then the state transition probability distribution $A=\left\{a_{i j} \mid i, j \in \mathcal{N}\right\}$ is given by

$$
a_{i j}=P\left[q_{t+1}=S_{j} \mid q_{t}=S_{i}\right] \text { for } i, j \in \mathcal{N} \text { and } t \in \mathcal{T}
$$

where every $a_{i j}$ coefficient (i.e., $a_{00}, a_{01}, a_{1 \mathrm{c}}, a_{i 1}$ ) is positive, and $\sum_{j \in \mathcal{N}} a_{i j}=1$ for $i \in \mathcal{N}$.

Now, we consider any one of the neural channels for which the observation is represented by $\mathrm{O}=\mathrm{O}_{1} \mathrm{O}_{2} \cdots O_{T}$. To avoid significant degradation due to any quantization process, we treat the PD representation to be continuous-valued and accordingly 
consider an HMM with continuous pdfs. However, the use of a continuous pdf requires some restrictions on its form so as to facilitate reestimation of the pdf pararisters (e.g., mean, variance) in a consistent manner. The pdf for each of the two states is maintained fixed regardless of when and how the state is reached. The most general representation of the pdf, for which a reestimation procedure exists [92], is used here. Each state $S_{j}$ is characterized by a continuous mixture pdf $b_{j}(x)$ of the form

$$
b_{j}(x)=\sum_{m \in \mathcal{M}_{L}} c_{j m} b_{j m}(x) \text { for } j \in \mathcal{N},
$$

where $\mathcal{M}_{L} \equiv\{1,2, \cdots, L\}$ with $L$ as the number of components in the mixture and $b_{j m}($.$) is any log-concave [92] or elliptically symmetric [97] density. The rationale$ behind choosing a mixture pdf and selecting the component pdf $b_{j m}($.$) to be log-$ concave or eiliptically symmetric is discussed later. In our present study, $b_{j m}($.$) is$ assumed to be a beta density function and can be written as

$$
b_{j m}(x)=\frac{\Gamma\left(d_{j m}+f_{j m}+2\right)}{\Gamma\left(d_{j m}+1\right) \Gamma\left(f_{j m}+1\right)} x^{d_{j m}}(1-x)^{f_{j m}} \quad \text { for } \quad d_{j m}, f_{j m}>0, j \in \mathcal{N}, m \in \mathcal{M}_{L},
$$

where $d_{j m}$ and $f_{j, \ldots}$ are the parameters associated with the density function. The bata fidf of (5.4) is suitable as the observations are continuous-valued between 0 and 1. The Appendix D shows that the beta density function satisfies the log-conacavity condition.

The observation probability density function $B$ is denoted as $B=\left\{b_{j}(x) \mid j \in \mathcal{N}\right\}$, where $b_{j}(x) d x$ is the probability of observing a value $O_{t}$ in state $S_{j}$ at clock time $t$. A coefficient $c_{j m}$ is the $m$-th component mixture gain in statc $S_{j}$ and the set $\left\{c_{j m} \mid j \in \mathcal{N}, m \in \mathcal{M}_{L}\right\}$ satisfies the stochastic constraint

$$
\sum_{m \in \mathcal{M}_{L}} c_{j m}=1 \text { for } j \in \mathcal{N} \text { with } c_{j m}>0 \text { for } j \in \mathcal{N} \text { and } m \in \mathcal{M}_{L}
$$

so that

$$
\int_{-\infty}^{\infty} b_{j}(x) d x=1, \quad j \in \mathcal{N}
$$

\subsection{Preliminaries}

In Section 5.2, an HMM has been defined by describing ...e complete parameter set of the model. The model is represented as $\lambda=(\pi, A, B)$, where $\pi$ is the state probability 


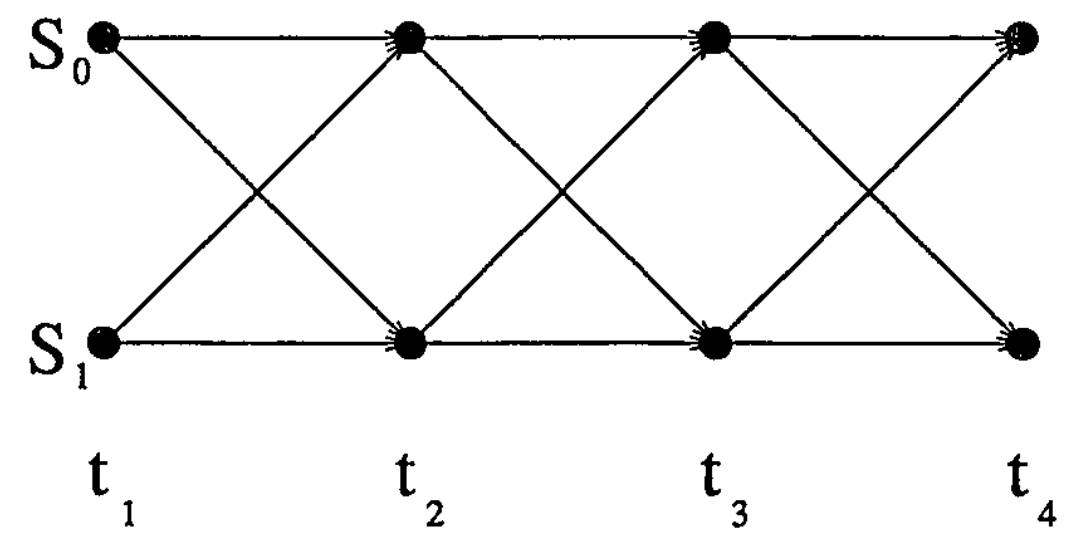

Figure 5.2: A two-state trellis diagram $\left(S_{0}\right.$ and $S_{1}$ denote the non-firing and firing states)

vector, $A$ is the state transition probability matrix and $B$ is a set of two $(N=2)$ continuous mixture pdfs, each with $L$ mixtures. In this section, we provide some preliminaries required for computing the degree of distortion (similarity) of a coded speech with reference to its original version. A forward and a backward likelihood variables and an auxiliary function are defined below.

\subsubsection{Forward and Backward Likelihood Variables}

Let us extend the integer set $\mathcal{T}$ to $\mathcal{T}^{+}$as $\mathcal{T}^{+} \equiv \mathcal{T}+\{T\}$. Following Baum [92], a forward likelihood variable $\alpha_{t}(i)$ is then defined as

$$
\alpha_{t}(i)=P\left(O_{1} O_{2} \ldots O_{t}, q_{t}=S_{i} \mid \boldsymbol{\lambda}\right), \quad \text { for } i \in \mathcal{N} \text { and } t \in \mathcal{T}^{+}
$$

which gives the probability of observing the partial sequence $O_{1} O_{2} \cdots O_{t}$ (until time $t$ ) and reaching the state $S_{i}$ at clock time $t$ given an HMM $\lambda$. Likewise, a backward likelihood variable $\beta_{t}(j)$ is defined as

$$
\beta_{t}(j)=P\left(O_{t+1} O_{t+2} \cdots O_{T} \mid q_{t}=S_{j}, \lambda\right), \quad \text { for } j \in \mathcal{N} \text { and } t \in \mathcal{T}
$$

which gives the probability of observing the partial sequence $O_{t+1} O_{t+2} \cdots O_{T}$ (from $t+1$ to the end) given state $S_{j}$ at time $t$ and a model $\boldsymbol{\lambda}$.

The forward likelihood variable $\alpha_{t}(i)$ is initialized as the joint probability of 
being in state $S_{i}$ at $t=1$ and an initial observation $O_{1}$, i.e.,

$$
\alpha_{1}(i)=\pi_{i} b_{i}\left(O_{1}\right), \quad i \in \mathcal{N} .
$$

With the help of the trellis diagram shown in Fig. 5.2, an iterative procedure is followed to compute the other forward likelihood variables from the initial one. Since $\alpha_{t}(i)$ is the probability of the joint ever: that $O_{1} O_{2} \cdots O_{t}$ are observed and the state $S_{i}$ is reached at clock time $t$, the product $\alpha_{t}(i) a_{i j}$ becomes the probability of the joint event that $O_{1} O_{2} \cdots O_{t}$ are observed and the state $S_{j}$ is reached at $t+1$ through the state $S_{i}$ at $t$. Summation of this product over the possible two states $S_{i}$ (for $i \in \mathcal{N}$ ) at time $t$ yields the probability of reaching state $S_{j}$ at $t+1$ with the corresponding partial observation sequence upto time $t$. Multiplication of the summed quantity by $b_{j}\left(O_{t+1}\right)$, the probability of observing $O_{t+1}$ at state $S_{j}$, results in the forward likelihood variable $\alpha_{t+1}(j)$ for time $t+1$. This evaluation procedure can be expressed by the following recurrence equation:

$$
\alpha_{t+1}(j)=\left[\sum_{i \in \mathcal{N}} \alpha_{t}(i) a_{i j}\right] b_{j}\left(O_{t+1}\right), \quad t \in \mathcal{T}, \quad j \in \mathcal{N}
$$

In a similar manner, let us now consider the backward variable $\beta_{t}(i)$. An initialization process arbitrarily defines

$$
\beta_{T}(j)=1, \quad j \in \mathcal{N} .
$$

Then, $\beta_{t}(i)$ is calculated recursively as follows:

$$
\beta_{t}(i)=\sum_{j \in \mathcal{N}} a_{i j} b_{j}\left(O_{t+1}\right) \beta_{t+1}(j), \quad t \in \mathcal{T}, \quad i \in \mathcal{N} .
$$

For a given model $\lambda, \beta_{t}(i)$ is the probability of observing the particular partial sequence from time $t+1$ to the end when it is known that the state $S_{i}$ is reached at time $t$. To compute this, it is evident from the trellis diagram of Fig. 5.2 that we need to consider both the states $S_{0}$ and $S_{1}$ at time $t+1$ accounting for the possible transitions from $S_{i}$ to $S_{j}$, the observation $O_{t+1}$ in state $S_{j}$ and also the partial observation sequence $O_{t+2} O_{t+3} \cdots O_{T}$ (being in state $S_{j}$ at timie $t+1$ ). 


\subsubsection{Auxiliary Function}

Following the concept of the Kullback-Leibler statistic, an auxiliary function $F\left(\lambda, \lambda^{\prime}\right)$ of two models $\lambda$ and $\lambda^{\prime}$, for a given observation vector $O$, can be defined [98] as

$$
F\left(\lambda, \lambda^{\prime}\right)=\sum_{Q \in \mathcal{N}^{T}} \sum_{M \in \mathcal{M}_{L}^{T}} P(O, Q, M \mid \lambda) \log P\left(O, Q, M \mid \lambda^{\prime}\right)
$$

with $\boldsymbol{Q}=q_{1} q_{2} \cdots q_{T}, M=m_{1} m_{2} \cdots m_{T}, q_{k} \in \mathcal{N}$ and $m_{k} \in \mathcal{M}_{L}$ for $k \in \mathcal{T}$. In the following, we show that if $F\left(\lambda, \lambda^{\prime}\right) \geq F(\lambda, \lambda)$, then $P\left(O \mid \lambda^{\prime}\right) \geq P(O \mid \lambda)$.

$$
\begin{aligned}
P(\boldsymbol{O} \mid \boldsymbol{\lambda}) \log \frac{P\left(\boldsymbol{O} \mid \boldsymbol{\lambda}^{\prime}\right)}{P(\boldsymbol{O} \mid \boldsymbol{\lambda})} & =P(\boldsymbol{O} \mid \boldsymbol{\lambda}) \log \sum_{Q \in \mathcal{N}^{T}} \sum_{M \in \mathcal{M}_{L}^{T}} \frac{P\left(\boldsymbol{O}, \boldsymbol{Q}, M \mid \boldsymbol{\lambda}^{\prime}\right)}{P(\boldsymbol{O} \mid \boldsymbol{\lambda})} \\
& =P(\boldsymbol{O} \mid \boldsymbol{\lambda}) \log \sum_{Q \in \mathcal{V}^{T}} \sum_{M \in \mathcal{M}_{L}^{T}} \frac{P(O, Q, M \mid \lambda)}{P(\boldsymbol{O} \mid \boldsymbol{\lambda})} \cdot \frac{P\left(\boldsymbol{O}, \boldsymbol{Q}, \boldsymbol{M} \mid \boldsymbol{\lambda}^{\prime}\right)}{P(\boldsymbol{O}, \boldsymbol{Q}, \boldsymbol{M} \mid \boldsymbol{\lambda})} \\
& \geq P(\boldsymbol{O} \mid \boldsymbol{\lambda}) \cdot \sum_{Q \in \mathcal{N}^{T}} \sum_{M \in \mathcal{M}_{L}^{T}} \frac{P(O, Q, M \mid \lambda)}{P(\boldsymbol{O} \mid \boldsymbol{\lambda})} \cdot \log \frac{P\left(\boldsymbol{O}, \boldsymbol{Q}, \boldsymbol{M} \mid \boldsymbol{\lambda}^{\prime}\right)}{P(\boldsymbol{O}, \boldsymbol{Q}, \boldsymbol{M} \mid \boldsymbol{\lambda})} \\
& =\left[F\left(\boldsymbol{\lambda}, \boldsymbol{\lambda}^{\prime}\right)-F(\boldsymbol{\lambda}, \boldsymbol{\lambda})\right] \geq 0
\end{aligned}
$$

with strict inequality except when $P(O, Q, M \mid \lambda)=P\left(O, Q, M \mid \lambda^{\prime}\right)$. In the above, the fact that $\log x$ is strictly concave for $x>0$ (since $d^{2} / d x^{2}(\log x)=-x^{-2}<0$ ) has been used. The first inequality is the well-known Jensen's inequality whereas the second one is true by hypotinesis. If the current model is ciefined as $\lambda=(\pi, A, B)$ and a reestimated model is $\lambda^{\prime}=\left(\pi^{\prime}, A^{\prime}, B^{\prime}\right)$; then either the initial model $\boldsymbol{\lambda}$ defines a critical point of the likelihood function (in that case $\lambda^{\prime}=\lambda$ ), or the model $\lambda^{\prime}$ is better than the model $\lambda$ in a sense that the observation sequence $O$ is more likely to have been generated by $\lambda^{\prime}$. From (5.14), we observe that the maximization of $P(O \mid \lambda)$ implies maximization of the auxiliary function; and hence a critical point of the auxiliary function gives an estimate about the HMM parameters.

\subsection{Distortion Measure Methodology}

An original speech segment and its coded version are passed through the cochlear model to obtain the PD representations. For each of these segments, the PD observations are sequences of 64-dimensional vectors corresponding to sixty-four neural 
channels. A hidden Markov model is associated with each of the channels and the parameters are estimated from the $P D$ observation sequence produced by the original speech segment. In a sense, all the sixty-four HMMs are 'trained' with the pertinent observation vectors corresponding to the original speech segment. Then, for the same speech segment, the observations from all the coded specch signals are 'matched' against the derived HMMs to compute the relative coder distortions. Now we describe the exact procedures for the model parameter estimation as well as the likelihood computation.

\subsubsection{Parameter Estimation}

There is no optimal way of estimating the model parameters from any finite-length observation sequence. Since the closed-form maximum likelihood is not possible, the HMM parameters are (re)estimated iteratively starting from an initial estimate. To solve this problem, Baum-Welch reestimation algorithm [99] is used here. $\Lambda \mathrm{n}$ application of this algorithm is equivalent to solving a mathematical optimization problem for obtaining the maximum likelihood estimates of the HMM parameters. The scheme for estimating the HMM parameters is based on the maximization of the probability of the observation sequence given a model. This algorithm is quite powerful as it ensures a monotonic increase in the likelihood with the successive iterations of the algorithm [92].

Let us now consider the calculation of $P(O \mid \lambda)$, the probability of the observation sequence $O$ given the model $\lambda$. Assuming the statistical independence of observations, for every given state sequence $Q=q_{1} q_{2} \cdots q_{T}$, the probability of observing $\boldsymbol{O}$ can be written as $P(\boldsymbol{O} \mid \boldsymbol{Q}, \boldsymbol{\lambda})$, where

$$
P(O \mid Q, \lambda)=b_{q_{1}}\left(O_{1}\right) b_{q_{2}}\left(O_{2}\right) \cdots b_{q_{T}}\left(O_{T}\right) .
$$

The probability of the occurrence of such a state sequence $Q$ is given as

$$
P(Q \mid \lambda)=\pi_{q_{1}} a_{q_{1} q_{2}} a_{q_{2} q_{3}} \cdots a_{q_{T-1} q_{T}} .
$$

Using (5.15) and (5.16), $P(O \mid \lambda)$ can be computed as

$$
P(\boldsymbol{O} \mid \boldsymbol{\lambda})=\sum_{Q \in \mathcal{N}^{T}} P(\boldsymbol{O} \mid \boldsymbol{Q}, \boldsymbol{\lambda}) P(\boldsymbol{Q} \mid \boldsymbol{\lambda}) .
$$


The global density function of (5.17) with the state density defined by (5.3) can be rewritten as

$$
\begin{aligned}
P(\boldsymbol{O} \mid \boldsymbol{\lambda}) & =\sum_{Q \in \mathcal{N}^{T}} \pi_{q_{1}} \prod_{t \in \mathcal{T}^{+}}\left[a_{q_{t} q_{t+1}}\left\{\sum_{m \in \mathcal{M}_{L}} c_{q_{i} m} b_{q_{t} m}\left(O_{t}\right)\right\}\right] \\
& =\sum_{Q \in \mathcal{N}^{T}} \sum_{m_{1} \in \mathcal{M}_{L}} \sum_{m_{2} \in \mathcal{M}_{L}} \cdots \sum_{m_{T} \in \mathcal{M}_{L}}\left[\pi_{q_{1}} \prod_{t \in T^{+}} a_{q_{t} q_{t+1}} c_{q_{t} m_{t}} b_{q_{t} m_{t}}\left(O_{t}\right)\right]
\end{aligned}
$$

assuming the parameter $a_{q_{T} q_{T+1}}=1$. The direct computation of $P(O \mid \lambda)$ as given by $(5.18)$ involves enumerating every possible state sequence of length $T$. Instead, we exploit the trellis structure and use (5.10) and (5.12) for the forward and the backviard likelihood parameters. In order to describe the procedure for an iterative update of the HMM parameters, we define a set of transition likelihood variables $\left\{\xi_{t}(i, j) \mid i, j \in \mathcal{N}, t \in \mathcal{T}\right\}$ as

$$
\xi_{t}(i, j)=P\left(\boldsymbol{O}, q_{t}=S_{i}, q_{t+1}=S_{j} \mid \boldsymbol{\lambda}\right)
$$

which gives the probability of observing the particular sequence $O$, and being in the state $S_{i}$ at time $t$ and the state $S_{j}$ at time $t+1$ given the model. From the trellis diagram of Fig. 5.2 , it can be noted that $\xi_{t}(i, j)$ can be written as

$$
\xi_{t}(i, j)=\sum_{m \in \mathcal{M}_{L}} \alpha_{t}(i) a_{i j} c_{j m} b_{j m}\left(O_{t+1}\right) \beta_{t+1}(j)
$$

We note the following relationships among the three likelihood variables as defined in (5.10), (5.12) and (5.19):

1. A product of the forward and the backward likelihood variables for any clock time $t$ is shown, using (5.3) and (5.12), equal to the sum of the transition likelihood variable $\xi_{t}(i, j)$ over the index $j$.

$$
\begin{aligned}
\alpha_{t}(i) \beta_{t}(i) & =\alpha_{t}(i)\left[\sum_{m \in \mathcal{M}_{L}} \sum_{j \in \mathcal{N}} a_{i j} c_{j m} b_{j m}\left(O_{t+1}\right) \beta_{t+1}(j)\right] \\
& =\sum_{j \in \mathcal{N}} \xi_{t}(i, j) .
\end{aligned}
$$

2. Using (5.10) and (5.12), it is shown that a sum of the product of the forward and the backward likelihood variables, i.e., $\alpha_{t}(i) . \beta_{t}(i)$ over $i$ is independent of 
the time index $t$.

$$
\begin{aligned}
\sum_{j \in \mathcal{N}} \alpha_{t+1}(j) \beta_{t+1}(j) & =\sum_{j \in \mathcal{N}}\left[\sum_{i \in \mathcal{N}} \sum_{m \in \mathcal{M}_{L}} \alpha_{t}(i) a_{i j} c_{j m} b_{j m}\left(O_{t+1}\right)\right] \beta_{t+1}(j) \\
& =\sum_{i \in \mathcal{N}} \alpha_{t}(i)\left[\sum_{j \in \mathcal{N}} \sum_{m \in \mathcal{M}_{L}} a_{i j} c_{j m} b_{j m}\left(O_{t+1}\right) \beta_{t+1}(j)\right] \\
& =\sum_{i \in \mathcal{N}} \alpha_{t}(i) \beta_{t}(i) \quad \text { for } t \in \mathcal{T}
\end{aligned}
$$

3. Using (5.11) and applying (5.22) recurrently, $P(O \mid \lambda)$ can be written as the sum of the terminal forward likelihood variables $\alpha_{T}(i)$ over $i$, i.c.,

$$
P(O \mid \lambda)=\sum_{i \in \mathcal{N}} \alpha_{T}(i)
$$

The logarithm of $P\left(O, Q, M \mid \lambda^{\prime}\right)$, the square brackeled term in (5.18), can be written as

$$
\log P\left(\boldsymbol{O}, \boldsymbol{Q}, \boldsymbol{M} \mid \boldsymbol{\lambda}^{\prime}\right)=\log \pi_{q_{1}}^{\prime}+\sum_{t \in \mathcal{T}^{+}} \log a_{q_{t} q_{t+1}}^{\prime}+\sum_{t \in \mathcal{T}^{+}} \log c_{q_{t} m_{t}}^{\prime}+\sum_{t \in \mathcal{T}^{+}} \log b_{q_{t} m_{t}}^{\prime}\left(O_{t}\right) .
$$

It is seen that the HMM parameters $\pi^{\prime}, A^{\prime}$ and $B^{\prime}$ corresponding to the model $\lambda^{\prime}$ are segregated. Without any loss of generality, then the auxiliary function $F\left(\lambda, \lambda^{\prime}\right)$ of (5.13) can also be written in a separated form as

$$
\begin{aligned}
F\left(\boldsymbol{\lambda}, \boldsymbol{\lambda}^{\prime}\right)= & \sum_{Q \in \mathcal{N}^{T}} \sum_{M \in \mathcal{M}_{L}^{T}} P(O, Q, M \mid \lambda)\left\{\log \pi_{q_{1}}^{\prime}+\sum_{t \in \mathcal{T}^{+}} \log a_{q_{t} q_{t+1}}^{\prime}\right. \\
& \left.+\sum_{t \in \mathcal{T}^{+}} \log c_{q_{t} m_{t}}^{\prime}+\sum_{t \in \mathcal{T}^{+}} \log b_{q_{t} m_{t}}^{\prime}\left(O_{t}\right)\right\} .
\end{aligned}
$$

Since $F\left(\lambda, \lambda^{\prime}\right)$ is considered as the basis for the maximum likclihood optimization procedure, separability of the individual auxiliary function: as given in $\Lambda$ ppendix $\mathrm{E}$ simplifies the (re)estimation procedure. Individual maximization of the first three summands subject to the constraints

$$
\begin{gathered}
\sum_{j \in \mathcal{N}} \pi_{j}=1, \quad \pi_{j} \geq 0 \text { for } j \in \mathcal{N} . \\
\sum_{j \in \mathcal{N}} a_{i j}=1, \quad a_{i j} \geq 0 \text { for } i, j \in \mathcal{N} .
\end{gathered}
$$




$$
\sum_{m \in \mathcal{M}_{L}} c_{i m}=1, \quad c_{i m} \geq 0 \text { for } i \in \mathcal{N}, \quad m \in \mathcal{M}_{L}
$$

respectively, is well known. Each of the individual auxiliary functions has the same form $\sum_{j \in \mathcal{N}} u_{j} \log v_{j}$, which as a function of $\left\{v_{j} \mid j \in \mathcal{N}\right\}$ with the constraint $\sum_{j \in \mathcal{N}} v_{j}=$ 1 and $v_{j} \geq 0$ for $j \in \mathcal{N}$ attains a global maximum at the single point $v_{j}=u_{j} / \sum_{i \in \mathcal{N}} u_{i}$ for $j \in \mathcal{N}$. The initial probability $\bar{\pi}$ can be reestimated as

$$
\bar{\pi}_{i}=\frac{\alpha_{1}(i) \beta_{1}(i)}{\sum_{i \in \mathcal{N}} \alpha_{1}(i) \beta_{1}(i)}=\frac{\alpha_{1}(i) \beta_{1}(i)}{\sum_{i \in \mathcal{N}} \alpha_{T}(i)}, \quad \text { for } i \in \mathcal{N}
$$

which is the expected frequency in state $S_{i}$ at $t=1$. Similarly, the reestimation formula for $A$ results in a ratio of the expected number of transitions from state $S_{i}$ to state $S_{j}$ to the expected number of transitions out of state $S_{i}$, i.e.,

$$
\bar{a}_{i j}=\frac{\sum_{t \in \mathcal{T}^{+}} \xi_{t}(i, j)}{\sum_{t \in \mathcal{T}^{+}} \sum_{j \in \mathcal{N}} \sum_{m \in \mathcal{M}_{L}} \xi_{t}^{(m)}(i, j)}=\frac{\sum_{t \in \mathcal{T}^{+}} \xi_{t}(i, j)}{\sum_{t \in \mathcal{T}^{+}} \alpha_{t}(i) \beta_{t}(i)},
$$

where $\xi_{i}^{(m)}(i, j)$ is the probability of being in state $S_{j}$ at time $t+1$ and state $S_{i}$ at time $t$ with the $m$-th mixture component accounting for $O_{t}$, i.e.,

$$
\xi_{t}^{(m)}(i, j)=\xi_{t}(i, j) \cdot\left[\frac{c_{i m} b_{i m}\left(O_{t}\right)}{\sum_{i \in \mathcal{M}_{L}} c_{i l} b_{i l}\left(O_{t}\right)}\right] .
$$

with $b_{i m}\left(O_{t}\right)$ as given by (5.3). $\bar{c}_{i m}$ is the ratio of the expected number of transitions out of state $S_{i}$ using the $m$-th mixture component to the expected number of total transitions out of state $S_{i}$. Thus, for $i \in \mathcal{N}$ and $m \in \mathcal{M}_{L}$, we get

$$
\bar{c}_{i m}=\frac{\sum_{t \in \mathcal{T}^{+}} \sum_{j \in \mathcal{N}} \xi_{t}^{(m)}(i, j)}{\sum_{t \in \mathcal{T}^{+}} \sum_{j \in \mathcal{N}} \sum_{m \in \mathcal{M}_{L}} \xi_{t}^{(m)}(i, j)}=\frac{\sum_{t \in \mathcal{T}^{+}} \sum_{j \in \mathcal{N}} \xi_{t}^{(m)}(i, j)}{\sum_{t \in \mathcal{T}^{+}} \alpha_{t}(i) \beta_{t}(i)} .
$$

The parameters set $\left\{d_{\text {im }} \mid i \in \mathcal{N}, m \in \mathcal{M}_{L}\right\}$ and $\left\{f_{\text {im }} \mid i \in \mathcal{N}, m \in \mathcal{M}_{L}\right\}$ can be calculated from the following two equations.

$$
\sum_{r=1}^{f_{i m}+1} \frac{1}{\left(d_{i m}+r\right)}=-\frac{\sum_{t \in \mathcal{T}^{+}} \sum_{j \in \mathcal{N}} \xi_{t}^{(m)}(i, j) \log \left(O_{t}\right)}{\sum_{t \in \mathcal{T}^{+}} \sum_{j \in \mathcal{N}} \xi_{t}^{(m)}(i, j)}
$$




$$
\sum_{r=1}^{d_{i m+1}} \frac{1}{\left(f_{i m}+r\right)}=-\frac{\sum_{t \in \tau} \sum_{j \in \mathcal{N}} \xi_{t}^{(m)}(i, j) \log \left(1-O_{t}\right)}{\sum_{t \in \mathcal{T}+} \sum_{j \in \mathcal{N}} \xi_{t}^{(m)}(i, j)},
$$

where the parameters $d_{i m}$ and $f_{i m}$ are assumed, for reducing computations, to take up integer values.

\subsubsection{Distortion Computation}

We now discuss the CHM measure methodology. At first, we obtain the PD observation sequences from the original signal. For each of the sixty-four neural channels, we consider these PD observations for a frame of $T$ consecutive clock limes. An HMM is associated with each of such channels and the model parameters are determined starting from an initial estimate. Equations (5.29) to (5.34), derived based on the Baum-Welch algorithm, are used for estimating the model parameters. This technique iteratively chooses a 'better' model by maximizing $P\left(O_{n} \mid \lambda_{n}\right)$ where $O_{n}$ is the $n$-th channel PD observation sequence for the original speech. After a reasonable number of iterations, the algorithm is terminated and the final model is denoted as $\lambda_{n}^{(0)}$. Let the $n$-th channel PD observations for a corresponding coded speech be represented by $O_{n}^{(c)}$. Using (5.23), we compute $P\left(O_{n}^{(c)} \mid \lambda_{n}^{(o)}\right)$ for all the neural channels. This computation, in essence, evaluates the likelihood probability of the PD representation of the coded signal against the models derived from the PD representation of the original speech. We assume the neural channels to be independent and therefore the probability scores are multiplied. Upon taking logarithm, we obtain a similarity measure $M_{f}$ for the frame as

$$
M_{\mathcal{f}}=\sum_{n=1}^{64} \log P\left(O_{n}^{(c)} \mid \lambda_{n}^{(o)}\right) .
$$

Finally, a cochlear hidden Markovian (CHM) distortion measure value is defined by taking average of $M_{f}$ values over all the frames, negating it and also dividing it by 64 (i.e., $\mathrm{CHM}=-\bar{M}_{f} / 64$ ).

\subsubsection{Alternative Approaches}

Here, we suggest two other logical approaches for computing coder distortion although we have not carried out any tests with them. 


\section{State sequence approach}

One alternative method is to determine the 'optimal' state sequences associated with the PD observation sequences of an original speech as well as its coded version. An optimality criterion chooses the state $q_{t}$ that are individually most likely by maximizing the expected number of correct individual states. The inciividually most likely state $g_{t}$ at time $t$ is determined by computing

$$
q_{t}=\underset{i \in \mathcal{\Lambda}}{\operatorname{argmax}}\left[P\left(q_{t}=S_{i} \mid O, \lambda\right)\right]
$$

The bracketed term, i.e., the probability of being in state $S_{i}$ at time $t$, given the observation sequence $\boldsymbol{O}$ and the model $\lambda$, is written for the forward-backward technique in terms of the variables $\xi_{t}(i, j)$ as

$$
P\left(q_{t}=S_{i} ; O, \lambda\right)=\frac{\sum_{j \in \mathcal{N}} \xi_{t}(i, j)}{\sum_{i \in \mathcal{N}} \alpha_{T}(i)} .
$$

The solution simply determines the most likely state at every instant without any regard to the probability of occurrence for sequence of states. A distortion measure could be defined based on calculating the Hamming distance between the estimat $\mathrm{d}$ state sequences for the original and the coded speech signals. There is no unique way of selecting an 'optimality' criterion and the approach may even be modified to maximize the expected number of correct paths of pairs of states $\left(q_{t}, q_{t+1}\right)$ or triples of states $\left(q_{t}, q_{t+1}, q_{t+2}\right)$ etc.

\section{Model distance approach}

Another alternative is to estimate a model $\lambda^{(c)}$ from the PD observations of the coded speech frame exactly the way we have estimated the model $\lambda^{(0)}$ from the PD observation of the original speech frame. A nodel distance measure following the notion of discrimination information could be defined for comparing these pairs of HMMs [100]. One such measure form is

$$
D\left(\boldsymbol{\lambda}^{(\mathrm{c})}, \boldsymbol{\lambda}^{(0)}\right)=\sum_{n=1}^{64} \log P\left(\boldsymbol{O}_{n} \mid \boldsymbol{\lambda}_{n}^{(c)}\right)-\sum_{n=1}^{64} \log P\left(\boldsymbol{O}_{n} \mid \boldsymbol{\lambda}_{n}^{(0)}\right) .
$$

This measure is non-symmetric and a symmetrized version could be used in practice. 


\subsection{Practical Considerations}

A 'good' distortion measure should consider only the informativn a elevant to perceptuai events. However, the success of the measure also becomes heavily dependent on the accuracies of the implementation and the model description. Here, we discuss some practical aspects related to the evaluation of speech coders by the CHM measure.

\subsubsection{Computational Issues}

The forward probahility calculation is, in effect, based upon the trellis structure shown in Fig. 5.2. Since there are only two possible states at each time in the trellis, all the possible state sequences will rem rrge into one of these two nodes, regardless of the length of the observation sequence. At any time $t$, computation of $\alpha_{t}(j)$ involves only two previcus values of $\alpha_{t-1}(i)$ because each of the two grid points is reached from the same two grid points at the previous time slot. For computing each $\alpha_{t}(i)$ and $\beta_{t}(j)$, it requires on the order of $N^{2} T$ calculations, rather than $2 T N^{T}$ as required by the direct calculation.

Another important issue is that computing the likelihood variables involves multiplication of many terms having values smaller than 1 . In a recursive procedure, each term of these variables starts to diminish towards zero exponentially and thus the number representation goes below the precision range of any machine. To circumvent this problem, the likelihood and other variables are multiplied by constants known as scaling coefficienis [101]. The scaling procedure is not applied at every clock time, but once every few clock times.

\subsubsection{Initial Estimates for HMM Parameters}

Since a convergent reestimation procedure exists for the continuous mixture model considered here, it is theoretically possible to have arbitrary initial estimates for the HMM parameters obeying the stochastic constraints. The restimaition equations provide values for the HMM parameters corresponding to a local maximum of the likelihood function. The choice of 'good' initial estimates is thus important in making the convergence faster or ensuring the local maximum to be the global maximum of 
the likelihood function. In fact, some of the parameters may be very sensitive to the initial estimates [102].

\subsubsection{Training Data and Iterations}

The PD observation sequence used for 'training' the models has a finite length and this causes problem in determining the HMM parameters via reestimation method. An insufficient number of occurrences of different model events does not truely portray the real scenario and therefore we have to have sufficiently long training data. On the other hand, we wint the model parameters to be fixed for a specitic period and then vary depending on the new PD observations. The Baum-Welch estimation algorithm also needs several iterations before the convergence occurs.

\subsubsection{Mixture Processes}

It is an usual practice to approximate a $K$-dimensional correlated random process by a mixture of few uncorrelated, $K$-dimensional random processes. The number of mixture components is heavily dependent on the degree of correlation. By assuming mixture uncorrelated processes, we effectively reduce the number of parameters to be estimated and thus help making the estimates more reliable. The trade-off is clearly between the increased error in the approximation process and the increased reliability in the estimation rrocess.

\subsection{Experimental Results}

Before providing with the objective measure results, we describe the set-up procedure for some of the experimental parameters.

(i) We have 'trained' and 'matched' the HMMs with speech frames of 480 samples. For $N=2$ and $T=480$, only about 1920 computations were needed since the algorithm used was based on trellis structure.

(ii) The scaling procedure was used ist at every instant, but after every ten clock times. 
(iii) Although the length of the PD sequences over which the training and matching were done is 480 , we overlapped each such frame with the previous frame by $50 \%$. In other words, the observation window was shifted by 240 samples for dealing with each new model. This has allcwed having sufficiently long training data and also has facilitated the models not to change the parameters drastically.

(iv) In our experiment, we have chosen models with three mixture components (i.e., $M=3$ ). This has appeared to be a reasonable choice for making trade-off between the accuracy of modeling the histogram and the number of parameters to be estimated.

(v) Based on the psychoacoustic data, we have assumed the initial transition probability from a non-firing state to another non-firing state is 0.8 and that from a firing state to another firing state is 0.2 . In accordance with this, the initial state probabilities were chosen to be 0.8 for non-firing state $\left(S_{0}\right)$ and 0.2 for firing state $\left(S_{1}\right)$.

(vi) The initial estimates for the beta pdf parameters $\left\{d_{i m}\right\}$ and $\left\{f_{i m}\right\}$ were chosen in such a fashion that the corresponding mean values were $0.25,0.50$ and 0.75 for $i \in \mathcal{N}$. The weighting factors $\left\{c_{i m}\right\}$ were all assumed to be equal (i.e., 0.33 ) initially.

(vii) For any particular neural channel, the final estimate of HMM parameters obtained for a speech frame was considered as the initiai estimate of the parameters for the subsequent frame.

(viii) While solving the simultineous equations of (5.33) and (5.34), the $\left\{d_{i m}\right\}$ and $\left\{f_{i, m}\right\}$ parameters were allowed to take up integral values between 1 and 10 . Since the exact solution could not be found, we have determined the parameter values by choosing the best pair which minimizes the sum of the square errors. One more constraint imposed on the parameters was that the mean values (given by $d_{i m} /\left(d_{i m}+\right.$ $\left.f_{i m}\right)$ ) for three different mixture components have been kept confined to three different regions-one between 0 and $1 / 3$, the second between $1 / 3$ and $2 / 3$; and the third between $2 / 3$ and 1 . This also reduced the search for best solution by making some combinations of the parameter values to be invalid.

(ix) For model parameter estimations, we have made 30 iterations each time. 


\begin{tabular}{ccccccccccccc}
\hline Sent. & \multicolumn{1}{c}{ C1 } & \multicolumn{1}{c}{ C2 } & \multicolumn{2}{c}{ C3 } & \multicolumn{2}{c}{ C4 } & \multicolumn{2}{c}{ C5 } & \multicolumn{2}{c}{ C6 } \\
\hline & $S$ & $H$ & $S$ & $H$ & $S$ & $H$ & $S$ & $H$ & $S$ & $H$ & $S$ & $H$ \\
\hline M1 & 5.75 & 195 & 4.92 & 225 & 4.17 & 336 & 2.58 & 358 & 2.58 & 365 & 1.00 & 420 \\
\hline M2 & 5.50 & 250 & 5.17 & 231 & 4.25 & 280 & 2.75 & 310 & 2.25 & 390 & 1.08 & 414 \\
\hline F1 & 5.67 & 209 & 5.00 & 263 & 4.25 & 300 & 2.33 & 389 & 2.58 & 371 & 1.17 & 430 \\
\hline F2 & 5.67 & 220 & 5.00 & 276 & 3.91 & 347 & 2.67 & 378 & 2.50 & 312 & 1.25 & 398 \\
\hline
\end{tabular}

Table 5.: Subjective and objective measure values for six coded signals with reference to the corresponding original speech utterances (' $S$ ' gives the average subjective ranking scores and ' $H$ ' denotes the cochlear hidden Markov measure with single channel (CHM-SC))

\begin{tabular}{ccccccccccccc}
\hline Sent. & \multicolumn{1}{c}{$\mathrm{C} 1$} & \multicolumn{2}{c}{$\mathrm{C} 2$} & \multicolumn{2}{c}{ C3 } & \multicolumn{2}{c}{ C4 } & \multicolumn{2}{c}{ C5 } & \multicolumn{2}{c}{ C6 } \\
\hline & $S$ & $H$ & $S$ & $H$ & $S$ & $H$ & $S$ & $H$ & $S$ & $H$ & $S$ & $H$ \\
\hline M1 & 5.75 & 146 & 4.92 & 188 & 4.17 & 256 & 2.58 & 314 & 2.58 & 320 & 1.00 & 408 \\
\hline M2 & 5.50 & 161 & 5.17 & 179 & 4.25 & 238 & 2.75 & 287 & 2.25 & 346 & 1.08 & 398 \\
\hline M3 & 5.75 & 157 & 5.17 & 183 & 4.00 & 261 & 2.58 & 310 & 2.33 & 304 & 1.17 & 401 \\
\hline M4 & 5.00 & 196 & 5.67 & 152 & 4.25 & 230 & 2.50 & 326 & 2.58 & 311 & 1.00 & 412 \\
\hline M5 & 5.75 & 138 & 5.17 & 170 & 3.83 & 277 & 2.67 & 301 & 2.50 & 335 & 1.00 & 421 \\
\hline M6 & 5.58 & 163 & 5.25 & 186 & 3.83 & 265 & 2.75 & 292 & 2.42 & 319 & 1.17 & 392 \\
\hline F1 & 5.67 & 154 & 5.00 & 182 & 4.25 & 244 & 2.33 & 326 & 2.58 & 307 & 1.17 & 416 \\
\hline F2 & 5.67 & 159 & 5.00 & 192 & 3.91 & 270 & 2.67 & 296 & 2.50 & 310 & 1.25 & 386 \\
\hline F3 & 5.50 & 170 & 5.17 & 177 & 4.25 & 221 & 2.50 & 319 & 2.25 & 352 & 1.33 & 381 \\
\hline F4 & 5.41 & 169 & 5.25 & 174 & 4.17 & 238 & 2.75 & 281 & 2.17 & 361 & 1.25 & 399 \\
\hline F5 & 5.50 & 162 & 5.50 & 155 & 3.83 & 272 & 2.33 & 330 & 2.50 & 304 & 1.33 & 373 \\
\hline F6 & 5.67 & 156 & 4.83 & 202 & 4.08 & 263 & 3.08 & 322 & 2.17 & 348 & 1.17 & 391 \\
\hline
\end{tabular}

Table 5.2: Subjective and objective measure values for six coded signals with reference to the corresponding original speech utterances (' $S$ ' gives the average subjective ranking scores and ' $H$ ' denotes the cochlear hidden Markov measure with three channels $(\mathrm{CHM}-\mathrm{TC}))$ 
In this work, we have followed two strategies for computing coder distortions. Let us now consider determining the model parameters for the $n$-th neural channel. In the first strategy, while training the model, we have used only the $n$-th channel $\mathrm{PD}$ observation sequence corresponding to the original speech. We call this strategy as CHM-SC (with single channel). Table 5.1 shows subjective and objective measure values for six coded signals with reference to the original speech utterance. We tabulate here measure values for only four utterances. The CHM-SC measure was found to be not very satisfactory in ranking coded signals.

It has been our understanding that the training data length was not sufficient in the CHM-SC strategy to make a reliable estimate for the model parameters. Therefore, we formulated a new strategy where three adjacent channels-the $(n-1)$-th, $n$-th and $(n+1)$-th channel PD sequences were used in alternate manners for training. This strategy has been termed as the CHM-TC (with three channels). Table 5.2 provides subjective and CHM-TC measure values ior all the twelve utterances given in the Appendix C.

\begin{tabular}{rcrrrr}
\hline Coded Speech & Measure & \multicolumn{4}{c}{ Sample Delays } \\
\hline & & Zero & One & Two & Three \\
\hline oakf8f & SNR (w/o scaling [dB]) & 8.724 & 7.391 & 5.619 & 5.117 \\
\hline oakf8f & CHM-TC & 221 & 227 & 224 & 229 \\
\hline oakf8k & SNR (w/o scaling [dB]) & 9.178 & 7.503 & 6.108 & 7.027 \\
\hline oakf8k & CHM-TC & 319 & 321 & 326 & 323 \\
\hline
\end{tabular}

Table 5.3: The SNR and the cochlear hidden Markovian-threc channcls (CHM-TC) measure values with zero, one, two and three sample delays for the coded signal 'oakf8f' and 'oakf8k' with reference to the original speech sentence

For the CHM distortion measure values, we have computed the logarithm (natural) of the likelihood probability scores, negated them and averaged over all the channels and all the speech frames. Tables 5.1 and 5.2 provide these measure values where a low value implies a better perceptual quality. Already in Chapter 1 , we have noted that with the utterance $\mathrm{M} 1$, the $\mathrm{C} 4, \mathrm{C} 5$ coders and with the utterance $\mathrm{F} 5$, the $\mathrm{C} 1, \mathrm{C} 2$ coders were ranked same subjectively. The CHM-TC measure has found $\mathrm{C} 4$ coder for $\mathrm{M} 1$ and $\mathrm{C} 2$ coder for F5 to be slightly better than their counterparts. Other 
than these tie cases, the subjective and objective measures were not in conformance for the $\mathrm{C} 4, \mathrm{C} 5$ with the utterance M3.

The success of the CHM mecisure is quite comparable with that of the CDI measure. However, the primary two advantages of the CHM measure are that (i) ample provisions (selecting better initial estimates, carrying out more iterations etc.) exist for its improvement and (ii) it attempts to take time correlations into account and is fairly robust against few sample delays. Unlike most of the other distortion measures, the CHM measure performs quite well without an explicit time-alignment. Table 5.3 provides the SNR measure (without scaling) values as well as the CHM measure values with zero, one, two and three sample delays in the coded speech. The misaligned sample places are filled in with very small (approximately zero) values. It is observed that the sample delays do not affect the measure values considerably.

\subsection{Summary}

In ihis chapter, we have introduced a cochlear hidden Markovian (CHM) measure for computing coder distortion. We have attempted to capture the basics of neural firing events with simple hidden Markovian models where the occurrence of perceptualdomain observations and correlation among adjacent observations are modeled appropriately. A two-state (one each for firing and non-firing events), fully-connected HMM has been assuciated with each of the neural channels.

For computing coder distortions, at first, all the HMMs are 'trained' (i.e., the HMM parameters are estimated) with the PD observation derived from the original signal. The Iaum-Welch reestimation technique has been applied to derive the HMM parameters iteratively starting from an initial estimate. The PL observations obtained from the coded speech are 'matched' against these HMMs. A negated version of the log likelihood probability scores, averaged over all the speech speech frames and neural channels, has acted as the CHM distortion measure. This measure has shown promise by conforming appreciably with subjective evaluation results and also by exhibiting its robustness against coder delays. 


\section{Chapter 6}

\section{Applications in Coder Analysis}

\subsection{Introduction}

The earlier chapters have dealt with an auditory representation of specch and two distinct approaches for computing distortions by comparing these perceptual-domain parameters of a coded signal vis-a-vis its original version. We have evaluated the performances of speech coders with these measures and also have computed the ratedistortion functions for speech coding with one of them. Although we have not attempted to use our measure formulation in a closed-loop fashion in any specch coder, it may very well be possible to use it for 'populating' a codebook in the training phase and/or for 'selecting' an appropriate codebook entry in the transmission phase. $\Lambda$ typical speech coder has several components based on the features and its encoding parameters. For a low bit-rate speech coder, a proper bit allocation among these components plays a significant role in achieving a good perceptual quality for the coded speech. Thus, it would be helpful for the designer if there could be a way to assess the performances of these components in a separate manner.

State-of-the-art analysis-by-synthesis medium or low bit-rate speech coders comprise of a linear prediction filter to model the short-term spectrum, a pitch predictor to model the long-term periodicity and a stochastic codebook to represent the residual speech signal. In practice, some of these filter blocks and codebooks are often split into more than one components primarily to give different perceptual importance and also for computational reason. While transmitting, in the analysis phase, 
different synthesized signals are compared with the original signal by a fidelity criterion. A mean-square distortion criterion has been found to be unsatisfactory as it does not even address perhaps the most important perceptual event, namely the auditory masking. To address this issue, in the recent literature, various noise weighting schemes have been coupled with the mean-square distortion criterion and/or other noise shaping filters have been suggested.

A detailed analysis on a component-by-component basis for different coders with the same bit-rate is beyond the scope of this thesis. Nonetheless, in this chapter, we will outline two related applications of the proposed measures. The first part describes an analysis procedure for determining the the pitch frequency by examining the output space of the cochlear model and applying the CDI raeasure. With the help of this analysis, it is possible to compare the pitch information of the original signal and that retained in a coded version. This, in turn, could provide a feedback to the designer regarding the deficieiscy of the pitch filter component. In the second part, we corsiuuer a wideband speech coder which uses three-way split vector quantization for the LPC parameters and fractional pitch lag value in the pitch predictor. We apply the CDI as well as the CHM measures for studying the performances of different noise weighting methods as incorporated in this coder. The coder was designed by $\mathrm{K}$. Abboud [9] and the vvaluation of the noise weighting schemes was carried out jointly by this author and Abboud.

The remainder of this chapter is formatted as follows. Section 6.2 briefly reviews some of the existing pitch frequency estimation algorithms. Section 6.3 suggests an algorithmic approach, using the CDI measure form, for the pitch frequency determination from the PD representation of a speech signal. Section 6.4 describes a $11.2 \mathrm{kbps}$ code-excited linear prediction (CELP)-based wideband speech coder. Section 6.5 introduces some of the perceptual weighting schemes while Section 6.6 investigates their performances by the proposed objective measures. Thus, Sections 6.2 and 6.3 are related to the first application of pitch frequency estimation whereas Sections $6.4,6.5$ and 6.6 pertain to the second application of performance evaluation of perceptual weighting schemes implemented in a wideband coder. 


\subsection{Existing Pitch Estimation Algorithms}

One simple time-domain approach for the pitch estimation is to low-pass filter all the energy from the speech signal except the fundamental harnonic and then detect the zero-crossing rate (ZCR). The ZCR measure is related to the pitch (F0) by

$$
\mathrm{F} 0=\mathrm{ZCR} * f_{s} / 2
$$

where $f_{s}$ is the sampling frequency in samples/sec. The prime difficulty of this method is in the determiration of the cut-off frequency for the low-pass filter as it should be high enough to inciude the fundamental frequency from a high-pitched voice and low enough to exclude the first harmonic of a low-pitched voice. Morcover, the ZCR detects some time the first formant frequency rather than the $\mathrm{FO}$ if the former has sufficiently high energy. A windowed autocorrelation function (ACF) is often calculated by taking the product, of the speech sample $\{s[n]\}$ with its delayed version and passing it through a window filter $\{w[n]\}[103]$. In the pitch determination, the ACF $R_{n}[k]$ given by

$$
R_{n}[k]=\sum_{m=-\infty}^{\infty} s[m] w[n-m] s[m-k] w[n-m+k]
$$

is evaluated for $k$ ranging from the shortest possible period (e.g., $3 \mathrm{~ms}$ for a female voice) to the longest one (e.g., $20 \mathrm{~ms}$ for a male voice). Another alternative technique is to calculate the average magnitude difference function (AMDF) defined as

$$
\operatorname{AMDF}[k]=\sum_{m=-\infty}^{\infty}|s[m]-s[m-k]|
$$

which shows minimum for a $k$ value corresponding to the pitch period.

Frequency-domain techniques involve computing a windowed Fourier transform defined as

$$
S_{n}\left(e^{j \omega}\right)=\sum_{m=-\infty}^{\infty} s[m] e^{-j \omega m} w[n-m] .
$$

In a spectrogram, $S_{n}\left(e^{j \omega}\right)$ is plotted with the sample-time $n$ on the horizontal axis, the frequency $\omega$ on the vertical axis and the magnitude by darkness of the display. The pitch period can be detected by searching either the periodically-spaced vertical lines in a wideband spectrogram or the periodically-spaced horizontal lines in a narrowband spectrogram. For estimating the F0, a cepstral analysis technique has also been 
employed where the complex cepstrum exhibits sharp pulses spaced at intervals typical of the pitch periods.

Unlike the conventional techniques, an auditory model-based pitch determination technique works for varying pitch effects, and is robust against a wide range of distortions [104]. Eased on the duplex theory of the pitch perception, Lyon has published an 'auditory correlogram' [105]. Using this idea, Slaney et al. have recently proposed a perceptual pitch detector [104]. In this algorithm, a pre-processing step emphasizes the vertical structure in the correlogram, sums the value at each timedelay in the enhanced correlogram across all the frequencies and determines the pitch based on the location of the largest peak. Weintraub has used a cost-reduced correlogram version as a pitch tracker for his two voice sound separation experiments [106]. Seneff has used an auditory model and suggested a generalized synchrony detection (GSD) mechanism for detecting the pitch periodicities in the speech signal [107].

\subsection{Pitch Frequency Estimation}

Here we suggest an algorithm, using the CDI measure form, for estimating the pitch fundamental frequency.

A rectangular analysis window of $40 \mathrm{~ms}$ is chosen for a speech frame of $20 \mathrm{~ms}$ so that the successive windows overlap by $50 \%$ and at least two pitch periods are included in the analysis window. The output for each of the sixty-four neural channels is compared with itself delayed by $\tau$ samples ( $\tau$ up to $20 \mathrm{~ms}=160$ samples accounting for the lowest possible F0). With two sets $\mathcal{I} \equiv\{1,2, \cdots, 160\}$ and $\mathcal{K} \equiv\{1,2, \cdots, 64\}$, the comparison with the discrimination measure (e.g., with the $\left.D_{1}(P ; Q)\right)$ takes the form

$$
E_{k}(\tau)=\sum_{l \in \mathcal{I}} \sum_{j=1}^{2} p_{j k l} \log \left(\frac{p_{j k l}}{p_{j k(l+\tau)}}\right), \quad \text { for } \tau \in \mathcal{I}, k \in \mathcal{K} .
$$

In this way, from a two-dimensional time-place representation $\mathcal{A}$, we derive a crossentropogram $\mathcal{E}=\left\{E_{k}(\tau) \mid k \in \mathcal{K}, \tau \in \mathcal{I}\right\}$ which is also two-dimensional where the vertical direction corresponds to the channel $k \in \mathcal{K}$ and the horizontal direction corresponds to the sample delay $\tau \in \mathcal{I}$. To enhance the vertical structure of $\mathcal{E}$, a convolutional operator $O=[-1+2-1]$ is used to give $\mathcal{G}=\left\{G_{k}(\tau) \mid k \in \mathcal{K}, \tau \in \mathcal{I}\right\}$ 


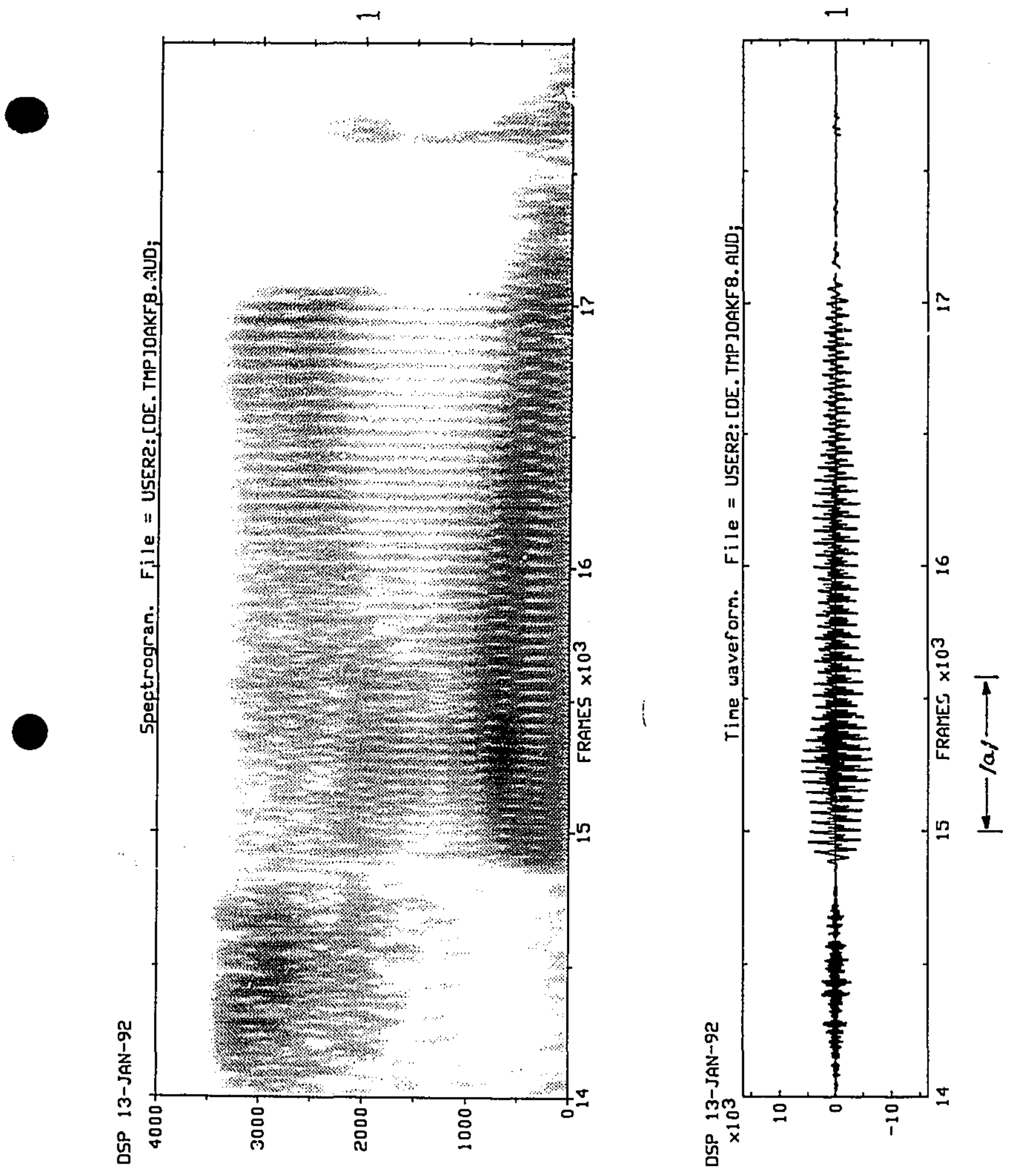

Figure 6.1: Time-domain waveform and spectrogram plot of the vowel /a/ in the word 'shade' (fomale voice) 
with

$$
G_{k}(\tau)=-E_{k}(\tau-1)+2 E_{k}(\tau)-E_{k}(\tau+1), \quad \text { for } \tau \in \mathcal{I}, \quad k \in \mathcal{K},
$$

where $E_{k}(0)=E_{k}(161)=0, \forall k \in \mathcal{K}$. An exponentiai weightivg set $\{w[k] \mid k \in \mathcal{K}\}$ of the form

$$
w[k]=e^{-\mathfrak{a}(N-k) / N}, \quad \text { for } k \in \mathcal{K}
$$

is defined where the number of channels $N$ is sixty-four and the decay factor $a$ is chosen to be 6 . The exponentially-weighted discrimination measure values for all tili. sample delays, summed over all the sixty-four channels, can then be written as.

$$
G(\tau)=\sum_{k \in \mathcal{K}} w\left[h_{\mathrm{j}} G_{k}(\tau), \quad \text { for } \quad \tau \in \mathcal{I}\right.
$$

The evidences from the higher harmonics are combined this way to make the pitch estimate robust. At the same time, the contributions from the formant frequencies are minimized by giving exponentially decaying weights to the higher frequency channels. In this flattened one-dimensional cross-entropogram $\mathcal{M}=\{G(\tau) \mid \tau \in \mathcal{I}\}$, the measure $G(\tau)$ shows the first significant dip at a $\tau$ value corresponding to the pitch period. Thus, an average $\mathrm{F} 0$ for a frame is calculated as

$$
\mathrm{F} 0=f_{s} *\left[\min _{\tau}\{\tau \in \mathcal{I}: G(\tau)<H\}\right]^{-1}
$$

with $f_{s}=8,000 \mathrm{~Hz}$ and an appropriate threshold $H$.

Fig. 6.1 shows the time-domain waveform and spectrogram plot for the word 'shade' (female voice). We execute our pitch estimation algorithm to determine the pitch period for one frame (160 samples starting from the sample number 15,000 ) of $/ a /$ in that word. In the one-dimensional cross-entropogram plot (using the directed divergence measure with $\alpha=1$ ) of Fig. 6.2, the first dip in $G(\tau)$ is observed at $\tau=40$ samples (equivalently, $5 \mathrm{~ms}$ ) with an $H$ value, say, -30 units. The perceptual pitch period is thus calculated to be $200 \mathrm{~Hz}$. It is expected that this scheme, due to merging of information from all the channels, could estimate the pitch correctly even when the 'fundamental frequency' component is fiitered out from the original signal. However, the selection of $I I$ may become more stringent. A post-processor may be used for the pitch estimates of successive frames to correct any serious error, e.g., pitch doubling or halving. 


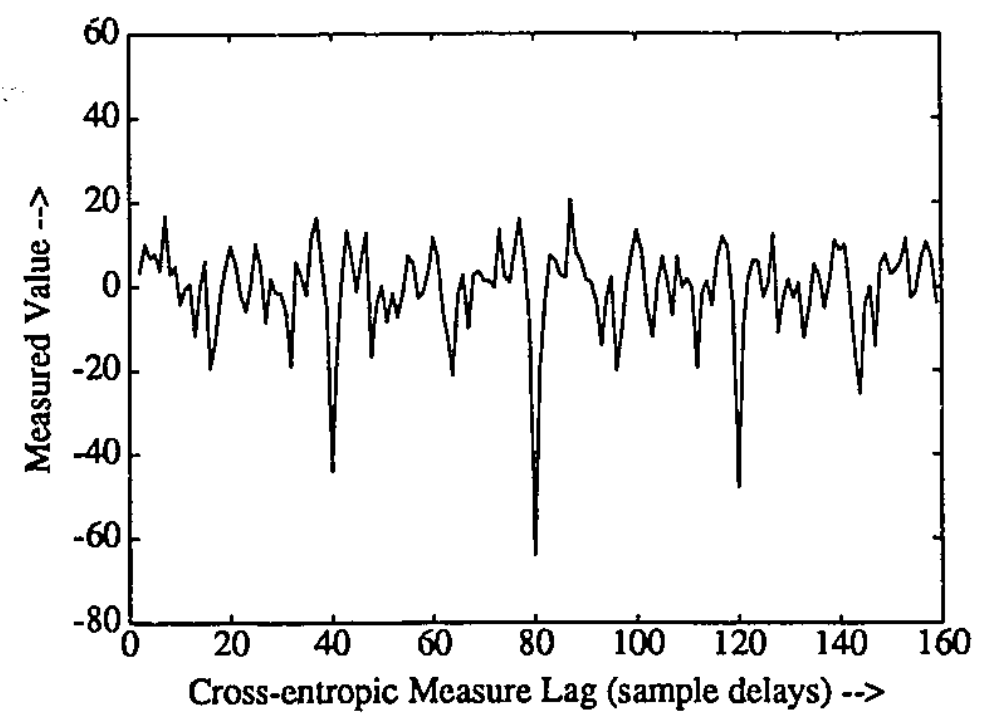

Figure 6.2: One-dimensional cross-entropogram (directed divergence with $\alpha=1$ ) for one particular frame (160 samples starting from the sample number 15,000$)$ of $/ a /$ in the word 'shade'

\subsection{Wideband Coder Architecture}

In the second application, our intention is to examine the performances of some of the noise shaping schemes generally incorporated in a medium or low bit-rate speech coder. Here, we consider a $11.2 \mathrm{kbps}$ CELP-based wideband speech coder. A wideband $(50-7,000 \mathrm{~Hz})$ speech shows superiority in the perceived quality over a narrowband $(200-3,400 \mathrm{~Hz})$ speech. This stems from the fact the added low frequencies increase the naturalness of a voice while the added high frequencies make the speech sound more intelligible, especially for fricative sounds. Obviously, more bits are required to code the additional information which leads us to have a trade-off between preserving acceptable speech quality of the reconstructed signal and maintaining a relatively low operating bit-rate. However, here our objective is not efficient coding; but assessing the effectiveness of the perceptual weighting schemes.

The CELP analysis-by-synthesis speech coders treat the input speech samples on a frame-by-frame basis. Linear prediction operations are used to exploit the fact that the speech exhibits a high degree of intersample correlations-correlation observed between adjacent samples (near-sample redundancy) and also, for voiced speech, cor- 
relation between samples separated by the pitch period (far-sample redundancy). Generally, a CELP coding technique consists of three basic functions- (i) short-term prediction (in the analysis phase) to determine the LPC (or equivalent) coefficients, (ii) pitch search (in the synthesis phase) to calculate the pitch lag (i.e., the pitch period in samples) and the pitch coefficient (i.e., the corresponding gain parameter) values and (iii) codebook search (in the synthesis phase) to determine the index of an excitation waveform and the associated scale factor.

Accordingly, two codebook indices and two quintized gain values are determined along with the formant predictor coefficients. In fact, the CELP coder does not directly need an analysis stage; ideally, the formant synthesis filter could also bc optimized for each candidate excitation waveform. However, the formulation of an optimal (in a mean-square sense) formant synthesis filter leads to a highly nonlinear set of equations which is not amenable to solution. Thus, a formant synthesis filter is generally implemented as the inverse of a formant filter determined in the analysis step. These parameters are selected in a systematic way for matching a synthesized speech to the original one with minimum error as defined by a disiortion measure. All of them are updated at regular intervals and transmitted over a communication channel in order to reconstruct the speech signal in the decoder side. A wide variety of wideband CELP-based algorithms has been proposed in the literature (e.g., [89]). In this work, we use a $11.2 \mathrm{kbps}$ wideband speech coder as designed by Abboud [9] and described below briefly.

\subsubsection{LSF-based Short-term Prediction}

For this wideband coder, a 16-th order LPC filter is chosen for the short-term prediction. The LPC parameters are determined by an autocorrelation method in which each frame of speech samples is multiplied by a Hamming window before getting filtered by the inverse formant filter. This method involves minimizing the residual signal (of the filtered version) energy and requires solving a set of Yule-Walker equations. The LPC parameters obtained are not well-suited for direct transmission because an error in any one parameter can cause the filter to become unstable and their wide dynamic range may make an efficient quantization practically impossible. Thus, they are transformed into a 'better-behaved' set of parameters such that the synthesis filter characteristics vary smoothly as a function of those parameters. 
The line spectral frequency (LSF) parameters represent the phase angle of an ordered set of poles on the unit circle. They are used quite often because they simplify the quantization procedure, ensure synthesis filter stability (if LSFs are ordered) and are closely relaied to the formant frequencies. An use of the LSF parameters has an added advantage of localized spectral sensitivity, i.e., that an error in one LSF only affects the synthesized spectrum near that frequency. An efficient technique for the computation of the LSFs is followed. The polynomial roots are determined by applying a Chebyshev transformation so as to map the upper semi-circle in the $z$-plane to the $[-1,+1]$ range and searching for sign changes in this interval [108].

These LSF parameters are quantized before transmission. In scalar quantization, each LSF coeficients are quantized individually while in the vector quantization (VQ), all the LSF coefficients are quantized together. For the same performance, the first one demands a high number of bits for quantization while the second one suffers from high complexity in terms of the amount of training data needed, the memory required and the number of computations involved. Here, a three-way split VQ technique for the LSF parameters is adopted. For each frame of $15.625 \mathrm{~ms}, 30$ bits are distributed among the 16 LSFs. They are divided into three subgroups-13 bits (for the first $8 \mathrm{LSFs}$ ), 9 bits (for the middle $4 \mathrm{LSFs}$ ) and 8 bits (for the last $4 \mathrm{LSFs}$ ). A training data of LSF vectors is used to construct three different optimal (at least in the local sense) codebook sets using the Linde-Buzo-Gray (LBG) algorithm [109].

During the transmission phase, an unquantized LSF vector is compared with the codebook entries for the LSF vectors. The algorithm chooses that codebook vector which minimizes a weighted-Euclidean LSF distance measure where the weighting factor considers the frequency sensitivity and also the LSF positions. A nested search technique is followed with priority given to the first LSF subgroup where most of the perceptual information prevails. The optimal first vector is combined with the second LSF codebcok entries to yield the second LSF vector; and finally, the optimal first and second vectors are combined with the third LSF codebook entries to generate the overall LSF vector. With a transmission of 30 bits per frame and an update rate of $64 \mathrm{~Hz}$, the operating rate for the short-term predictor is $1,920 \mathrm{bits} / \mathrm{sec}$. 


\subsubsection{Long-term Prediction with Fractional Delays}

The long-term linear predictor parameters are the pitch coefficient and the pitch lag. The pitch coefficient is a scaling factor related to the degree of waveform periodicity. It is zero for a signal without a periodic structure and is approximately one for steadystate voiced speech. The pitch lag tends to vary smoothly in the voiced segments with only occasional departure from the smooth trajectory [110]. However, in the unvoiced segments, the pitch lag tends to jump around. To avoid the problem of locking onto the correct pitch during the transition from silence to voiced speech, a good piich delay resolution should be maintained at all times during the analysis and synthesis stäges of the CELP coder.

The pitch coefficient values may be positive or negative. In general, the negative values tend to occur in the speech regions with low energy and the large positive values tend to occur in the transition regions (silence to speech). A restriction on the pitch period to be integer multiples of the sampling interval results in the partial destruction of the harmonic structure, especially in the high frequency regions. To increase the temporal resolution, non-integer (fractional) pitch lag values can be used. For this purpose, a multitap or a pseudo-multitap pitch prediction filter [111] can also be used. However, in this coder, a single-tap pitch predictor with fractional lag values is adopted. This is implemented by the use of interpolation and polyphase filters [112].

The pitch lag in wideband speech ranges from 40 to 320 samples with some delays occuring more often than others. With the use of 38,400 pitch subframes of $3.125 \mathrm{~ms}$ (five subframes in a formant frame of $15.625 \mathrm{~ms}$ ) each, a pitch delay distribution is generated. Based on this, a nonuniform distribution of non-integer delays is set up; the highest resolution is given to the pitch lags in the range of 71 to 100 while the lowest resolution is given to the end of the lag range. For each subframe, the pitch gain is represented by 4 bits whereas the pitch lag by 10 bits. Thus, with an update rate of $320 \mathrm{~Hz}$, the pitch parameters altogether require $4,480 \mathrm{bits} / \mathrm{sec}$.

\subsubsection{Residual Signal Codebook}

The residual signal codebook is filled up with codevectors containing sparse ternary elements. These excitations are generated by center-clipping and using a zero-mean unit-variance Gaussian sequence. The center-clipping threshold is set to \pm 1.2 in order 
to satisfy a specific percent (75\%) of sparsity inside the vector. All values between +1.2 and -1.2 in the Gaussian distribution are set to 0 , values greater than +1.2 are set to $+i$, and values less than -1.2 are set to -1 . The number of codewords is set to 1,024 requiring 10 bits for each subframe of $3.125 \mathrm{~ms}$. A 4 bit differential quantizer with a leaky predictor is used to code the differences in successive subframe magnitudes and an extra bit to code the sign. Thus, $4,800 \mathrm{bits} / \mathrm{sec}$ are required for residual signal representation with an update rate of $320 \mathrm{~Hz}$.

\subsection{Perceptual Noise Weighting}

At a low bit-rate, the mean-squared error criterion between the original and the reconstructed speech has been found to provide an unsatisfactory result. This indicates a requirement to shape the noise based on the auditory masking phenomenon in speech perception. In noise spectral shaping, the noise components at certain frequencies can only be diminished at the price of increased noise components at some other frequencies. At low bit-rates where the average noise level is quite high, it is difficult, if not impossible, to maintain noise below the masking threshold at all frequencies. The noise components in spectral valleys may exceed the threshold; nonetheless, they can be attenuated substantially by a postfilter used at the last stage of the decoding process. On the other hand, the postfiltering operation introduces distortion in the speech signal to some degree [113]. In this coder, the filter paramoters and the codebook entries are selected by minimizing a noise-weighted mean square error and/or using noise shaping filters. This section discusses three perceptually-weighted filtering schemes-(i) a simple noise weighting filter, (ii) a codebook shaping filter and (iii) an enhanced noise weighting filter. Although no postfilter is used here, it could be used with any of the above schemes. 


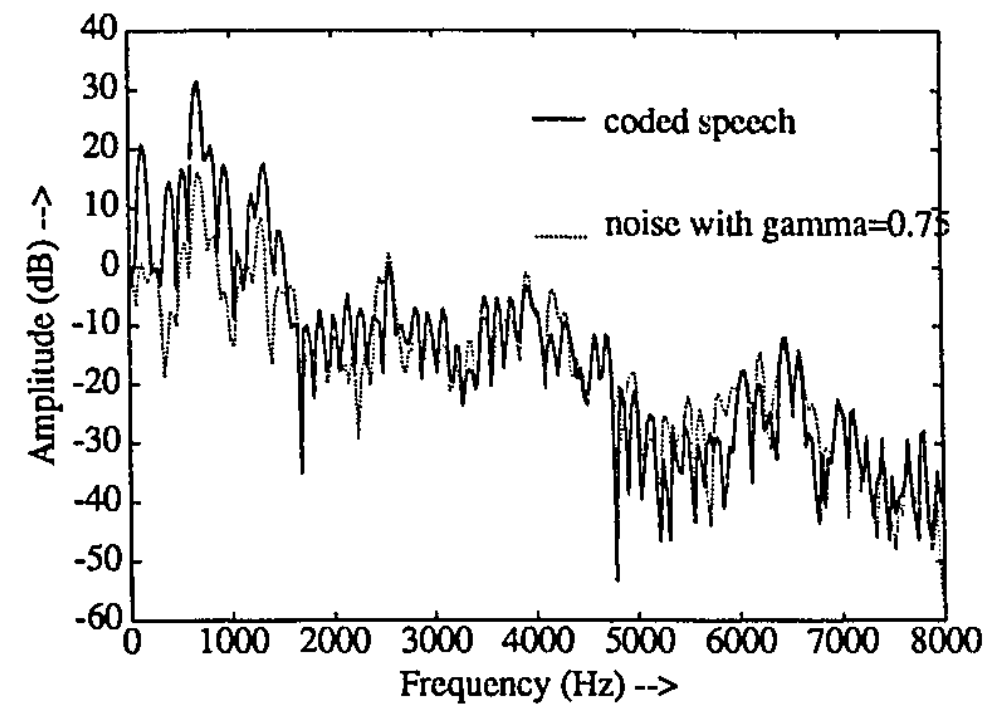

Figure 6.3: Noise weighting with $\gamma=0.75$.

\subsubsection{Simple Noise Weighting}

This auditory masking scheme is accomplished by minimizing a weighted mean-square error with a noise shaping filter $W(z)$ defined as

$$
W(z)=\frac{A(z)}{A^{\prime}(z / \gamma)}=\frac{1-\sum_{i=1}^{p} a_{i} z^{-i}}{1-\sum_{i=1}^{p} a_{i} \gamma^{i} z^{-i}}
$$

where $A(z)$ is the formant analysis filter with the predictor order $p$. The value of $\gamma(0<\gamma<1)$ is determined by the degree desired to de-emphasize the formant regions in the error spectrum. Decreasing the value of $\gamma$ moves the poles of the filter $1 / A(z / \gamma)$ inward and therefore increases the bandwidth of the poles of $W(z)$. A good value of $\gamma$ is 0.75 [9]. Fig. 6.3 shows the effect of using such a noise weighting filter in the reconstruction process of the input speech. The resulting noise level is no longer flat, but has the spectral shape of $W^{-1}(z)$ and therefore is boosted in the formant peaks and attenuated in the formant valleys. 


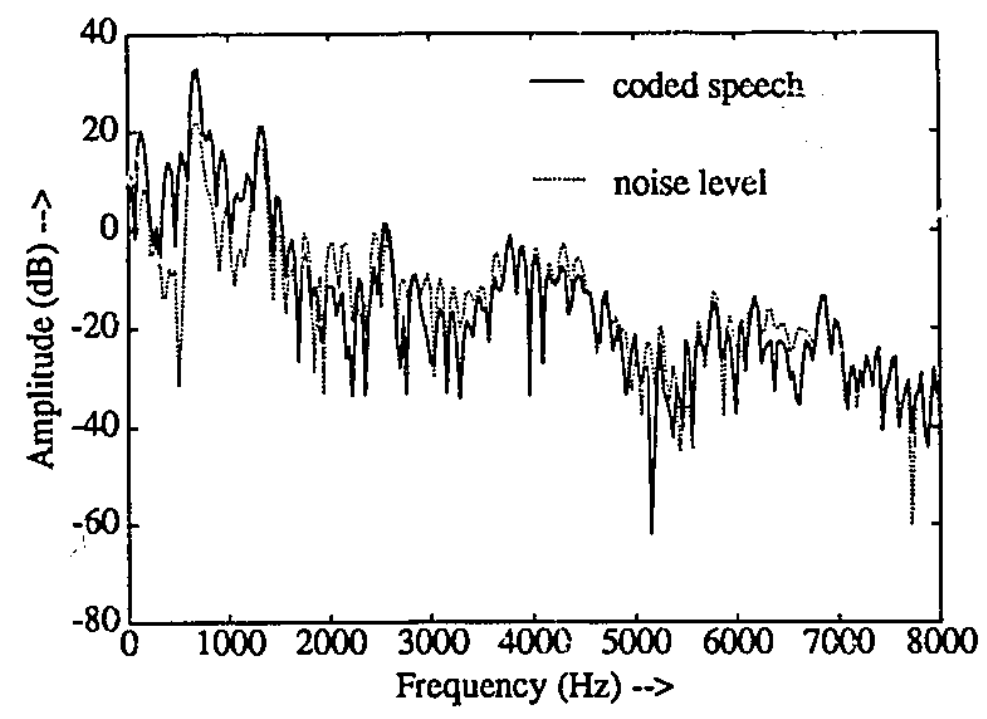

Figure 6.4: Noise level using codebook shaping filter.

\subsubsection{Codebook Shaping Filter}

In the second approach, an excitation codebook is cascaded with a shaping filter $F(z)$ to form a modified codebook structure. The filter $F(z)$, defined as [114]

$$
F(z)=\left(1-\mu z^{-1}\right) \frac{A\left(z / \gamma_{1}\right)}{A\left(z / \gamma_{2}\right)}
$$

is dynamically changed to control the statistical properties of the codebook in time and in frequency. The parameters $\gamma_{1}$ and $\gamma_{2}$ are de-emphasizing constants while $\mu$ is a parameter compensating for the spectral tilt. A differencer uses $\mu=1$, but an optimum preemphasis filter which maximizes the output spectral flatness measure has $\mu=\frac{r(1)}{r(0)}$, where $r(n)$ represents the autocorrelation sequence for the excitation signal before shaping. For unvoiced sounds, this fraction is relatively small and the effect of the preemphasis filter becomes negligible. On the other hand, for voiced sounds where $r(1)$ is very close to $r(0)$, the preemphasis filter acts almost as a differencer.

\subsubsection{Enhanced Noise Weighting}

The prime disadvantage of a simple noise weighting filter $W(z)$ is inadequate balancing of low and high frequency components due to interdependency of the tilt and 


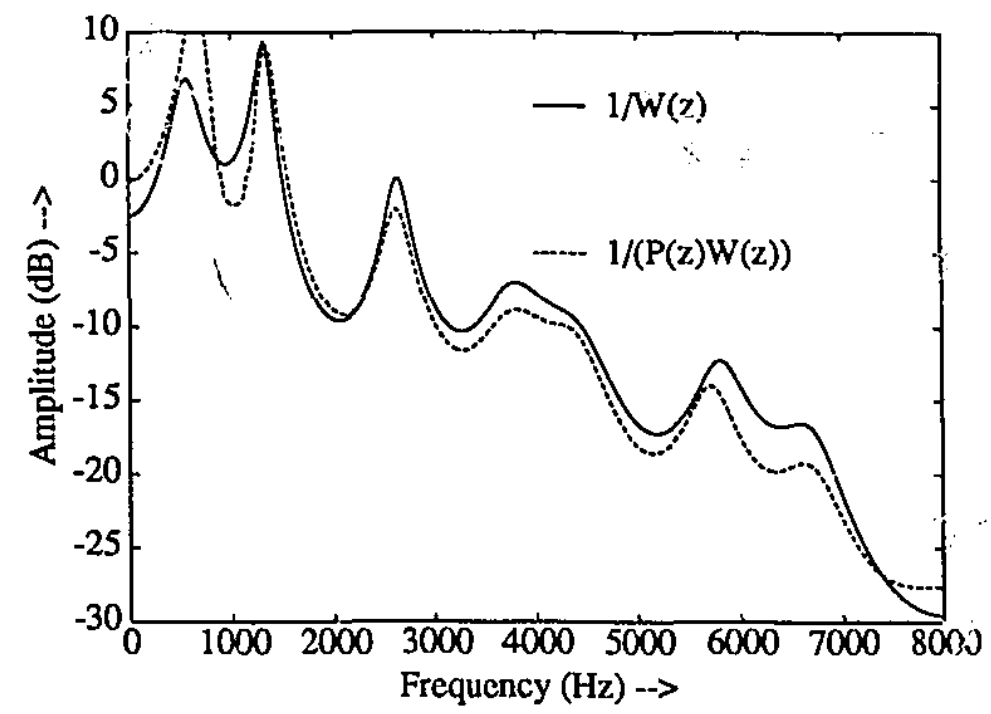

Figure 6.5: Performance of the weighting filter with $N=2$ and $\delta=0.7$.

formant parameters. An accurate modeling of one requires a sacrifice in modeling the other. An enhanced noise weighting technique [115] introduces a decoupling factor that results in an independent control of the tilt with respect to the formants. In general, the corresponding weighting filter is implemented as

$$
W_{N}^{\prime}(z)=W(z) P_{N}(z)=\frac{A(z)}{A(z / \gamma)} \frac{1}{1+\sum_{k=1}^{N} p_{k} \delta^{k} z^{-k}},
$$

where the coefficients $p_{k}$ are determined by an LPC analysis on the first $(N+1)$ correlation coefficients of the inverse filter $A(z)$ and the parameter $\delta$ controls the spectral tilt.

\subsection{Performance Evaluation}

We kept the wideband coder architecture in tact and applied different noise weighting schemes. In this second application, the primary contribution of this author, was in the performance evaluation of these schemes by the introduced objective measures [116]. The following seven configurations were used for the evaluation purpose.

1. no weighting, 
2. simple noise weighting vith $W(z)$,

3. simple noise weighting with $W(z)$ and also codebook shaping with $F(z)$,

4. enhanced noise weighting with $W_{3}^{\prime}(z)$,

5 . enhanced noise weighting with $W_{2}^{\prime}(z)$,

6. enhanced noise weighting with $W_{3}^{\prime}(z)$ and also codebook shaping with $F(z)$ and

7. enhanced noise weighting with $W_{2}^{\prime}(z)$ and codebook shaping with $F(z)$.

For the filter $W(z)$, the parameter $\gamma$ was chosen to be 0.75 . The filter $F(z)$ used parameters $\gamma_{1}=0.80, \gamma_{2}=0.95$ and an optimum $\mu$. The pardmeter $\delta$ for $W_{3}^{\prime}(z)$ was taken to be 0.5 whereas that for $W_{2}^{\prime}(z)$ was considered to be 0.7 .

$\therefore$\begin{tabular}{cccc}
\hline Config. & SNR $_{\text {seg }}(\mathrm{IB})$ & $\mathrm{CDI}$ & $\mathrm{CHM}-\mathrm{TC}$ \\
\hline 1 & 16.06 & 2.731 & 362 \\
\hline 2 & 12.89 & 2.684 & 284 \\
\hline 3 & 11.00 & 2.649 & 247 \\
\hline 4 & 11.90 & 2.543 & 183 \\
\hline 5 & 11.59 & 2.518 & 166 \\
\hline 6 & 9.69 & 2.606 & 212 \\
\hline 7 & 9.95 & 2.623 & 225 \\
\hline
\end{tabular}

Table 6.1: Distortion mcasures for different noise weighting configurations (the segmental SNR values $\left(\mathrm{SNR}_{\text {seg }}\right)$, the cochlear discrimination information measure values with $\alpha=1$ (CDI) and the cochlear hidden Markovian measure with three channels (CHM-TC) are tabulated)

For this part of the work with wideband speech, the cochlear model was extended to have eighty-five neural channel outputs. The transfer functions corresponding to all the filters are provided in [61]. It is worth mentioning that although sixty-four neural channels werc needed to cover from 0 to $4,000 \mathrm{~Hz}$, only an additional twenty-one channels were sufficient for covering the 4,000 to $8,000 \mathrm{~Hz}$ band. This is attributed to the fact that the center frequencies of the stages corresponding to these neural channels are logarithmically and not linearly placed. 
By evaluating the coded signal qua!ity with the CDI and CHM meastres, the effectiveness of the perceptual weighting schemes described in the previous section were assessed. The results with the $\mathrm{SNR}_{\text {seg }}$, the CDI (with $\alpha=1$ ) and the CHM measures are presented in Table 6.1. These experimental results indicate the following noints.

(a) As has been cïsrisssed earlier, the $\mathrm{SNR}_{\text {seg }}$ measure does not perform well as an objective criterion. Ironically, we observe here that the $\mathrm{SNR}_{\text {neg }}$ with the first configuration (no noise weighting filter) is highest whereas the perceptual quality measured subjectively as well as by the CDI and CHM measures is lowes among the others.

(b) The use of a codebook shaping filter $F(z)$ in combination with a simple noise weighting filter $W(z)$ yields better results in the perceptual domain than the configuration (1) with no shapiag filter. 'This is due to appropriate shaping of the excitation codevectors.

(c) The two-pole weighting filter $W_{2}^{\prime \prime}(z)$ exhibits better performance titan the three-pole weighting filter $W_{3}^{\prime}(z)$. The ihree-pole filter effectively boosts the high frequencies near the half sampling rate due to the presence of a real pole. However, this is achieved at the cust of a broadband increase in the level of distortion at lower frequencies. By getting rid of the real pole, the two-pole filter has been able to attain low level of distortion at lower frequencies while maintaining an acceptable level of high frequency noise.

(d) Among the configurations tested, the enharced noise weighting scheme with two-pole filter (i.e., the choice (5)) appears to be the best one. A pausible explanation for such a good performance of this configuration is that the weighting filter controls the tilt as weil as the formant parameters efficiently.

(e) We note that an integration of codebook shaping filter along with the enhanced noise weighting scheme (two or three poles), in fact, degrades the perceptual quality of the synthesized speech compared to the one generated by using only the enhanced noise weighting technique.

(f) In Table 6.1, we observe that the CDI and CHM measures are consistent with each other in ordering the coded signals. 
(g) For this part of the work, we did not conduct an informal listening test with all the twelve listeners; instead, only two of us (i.e., myself and Abboud) made a cursory subjective assessment. Our assessments agreed with the objective measure results. Despite the agreement made by the concerned objective measures about the relative superiority of the configuration (6) over (7), subjectively we assessed them to be of equal quality.

\subsection{Summary}

In this chapter, we have sketched applications of the proposed distortion measures in the analysis of speech coder components. Using the CDI measure form, the output space of the cochlear model was examined to estimate the pitch frequency. We carried out, although not presented here, some preliminary work for formant estimation [87] similar to the work presented in [107]. In another application, several noise weighting schemes were used in a wideband speech coder. As a coordinated work with $\mathrm{K}$. Abboud, the perceptual impacts of these techniques were studied with the CDI and CHM measures. We believe that this type of analysis could help the designer to study any particular section of the speech coder, adopt a new strategy and/or redistribute the available bits in a more efficient manner. 


\section{Chapter 7}

\section{Concluding Remarks}

In this dissertation, we have proposed two auditory distortion measures and investigated their performance in speech coder analysis and evaluation. Section 7.1 summarizes the key points of our work while Section 7.2 provides a future research direction related to our work.

\subsection{Summary of Our Work}

In Chapter 1, we have given a brief overview of speech coding techniques. The coding algorithms vary in the selection of features, in the parametric representation of features, in the quantization of parameters and in the computation of distortion. We have explained the importance of deriving an objective quality measure for specch coding. A 'good' measure could be used in the evaluation of specch coder performance, in the computation of rate-distortion function, in the analysis of speech coder components and also in the design of speech coder. In this dissertation, our purpose has been to introduce and investigate auditory distortion measures for coded speech.

In Chapter 2, we have reviewed some of the subjective and objective quality measures used in the speech coding area. Among the existing subjective measures, the MOS and DAM scores are more popular than the others. The time-domain objective measures such as the SNR and the segmental SNR are used widely for their simplicity even though they do not correlate well with subjective measures. We have studied numerous parametric distortion measures (e.g., the log likelihood ratio, the 
cepstral distance) based on all-pole speech synthesis models. We have also discussed two recently proposed psychoacoustically-motivated objective measures, namely the information index and the Bark spectral distance.

In this work, we have introduced two types of perceptual distortion measures for the purpose of speech coder evaluation. Towards this end, we have represented the speech signal onto a perceptual-domain using an auditory model. In Chapter 3, we have described the mechanism of auditory system and also analyzed some of the important psychoacoustic observations related to the speech perception. Among various functional auditory models, we have chosen Lyon's cochlear model. The outer-and-middle ear filter is modeled by a simple high-pass filter. The band-pass characteristics of the basilar membrane in the inner ear (cochlea) are simulated by sixty-four combinations of second-order notch filters and resonators. The activitics of the inner hair cells are mimicked by half-wave rectification process while those of the outer hair cells are imitated by the automatic gain control stages. Unlike many other models, Lyon's auditory model considers the temporal as well as spectral masking effects. The final representation of the cochlear model output is the probability-offiring information in the neural channels at the clock times.

In Chapter 4, we have introduced and studied a distortion measure, namely the cochlear discrimination information (CDI) measure, which compares the neural-firing information corresponding to an original speech and its coded version in a crossentropic sense. An insufficient knowledge about the exact neural firing processes has prompted us to use the probabilistic information of firing/non-firing in the comparison. We have investigated several variants of the CDI measure based on different types of entropy, the associated parameters and also the cross-entropic measure form. The effects of gain changes and sample delays etc. have also been studied. The directed divergence measure form based on the Rényi-Shannon entropy has shown very good performance by conforming strongly with informal subjective test in terms of ranking coded speech from six different coders. Subsequently, a rate-distortion analysis for speech coder has also been carried out with this measure. We have evaluated the rate-distortion function directly using the Blahut algorithm and also determined performances of four speech coders. We have observed that there is ample scope for improving the coded speech quality at a specified bit-rate.

We have suggested another approach towards formulating a perceptual distortion measure in Chapter 5. This method has used hidden Markovian model in an 
effort to capture the basic firing/non-firing process operative in the brain. We have considered two-state fully-connected model of order one for each neural channel; the two states of the model are corresponding to the firing and non-firing events. These models have been assumed to be stationary over a fixed duration (in our work, 480 sample times). The model parameters have been determined based on the PD observations corresponding to the original signal. The Baum-Welch optimization technique has been applied for the parameter estimation. Finally, the PD representations of the coded speech have been passed through the respective models so as to calculate the corresponding likelihood probabilities. The logarithms of these probability scores have been added and negated to give the cochlear hidden Markovian (CHM) distortion measure. This measure has shown promise by agreeing with subjective evaluation results to a large extent and also by demonstrating its robustness against sample delays.

Chapter 6 has outlined some of the possible applications of these measures in the analysis of speech coder components. The present-day analysis-by-synthesis medium or low bit-rate coders use several filters and codebooks. Keeping all but one component intact and having various configurations for the specific component under test, several coded versions could be synthesized for a speech utterance. As a first application, an algorithm for pitch frequency estimation has been suggested. This algorithm has involved examining the output space of the cochlear model with the CDI measure form and integrating information across channels. As a second application, different noise weighting schemes have been included in a wideband speech coder and their effect on performance has been evaluated by the CDI and CHM measures. An enhanced noise weighting scheme which controls the tilt as well as the formant parameters efficiently shows the best performance among the configurations.

While converting the time-domain speech signal into its corresponding PD representation by an auditory model, the resonating nature of the cochlea, the perceptual nonlinearity as well as the temporal and spectral masking effects have been considered. An inclusion of the spectral masking feature has allowed the probability-of-firing information in a particular neural channel at a specific clock time to depend not only on the strength of the gain-controlled signal of that channel but also on those of the other channels. Similarly, the same probability-of-firing information depends not only on the strength of the gain-controlled signal at that clock time but also on those at the other times. Thus, the PD representation for speech signal has exploited reasonably 
the interdependencies at the auditory periphery level.

In the CDI measure, we have compared element-by-element of the cochleagram matrices (whose elements are the probability-of-firing information) for the original and the coded speech signals. However, this measure has been found to be not very robust against the coder delays. Thus, estimating and removing time-delay between the original and the coded speech are, in some sense, necessary first steps in applying the CDI measure. The CHM neasure which has considered the temporal ordering in the firing pattern has shown a greater robustness against the coder delays. An explicit removal of the coder delays is not a necessity when the delays are confined to just a few samples. We believe that, even if the original and the coded speech signals are properly aligned, the CHM measure methodology is more powerful in the sense that it utilizes the contextual information present in the neural firing patterns.

\subsection{Future Research Directions}

In this section, we provide a future research direction by outlining some of the issues involved to improve this work.

\subsubsection{Improvement of Model Structure}

Lyon's auditory model which we have used in our work is, no doubt, a simplification of the complex behavior of the cochlea. The main simplification is in separating the interacting behaviors of the basilar membrane and the organ of Corti into noninteracting models-simple time-invariant filtering followed by a detection nonlinearity and an automatic gain control mechanism. A further refinement (e.g., [117]) of the model structure may improve the performance of the distortion measure. Some of the aspects for refinement are-fine-tuning the model parameters (e.g., $Q_{\text {ear }}, f_{e b}$ ), dynamically adjusting the $Q_{\text {ear }}$ value, making the model structure to be two- or threedimensional, incorporating the binaural feature etc. Many of these aspects may be important for other reasons such as localization of sound source etc. and thus may not contribute significantly in the distortion measure for coded speech. If we want to create a more biology-like condition by having a large number of neurons, it may become necessary to use a massively-parallel computer architecture based on 'connec- 
tionist' model or a neural network architecture based on Kohonen's self-organizing feature map [118].

\subsubsection{Reduction of Computational Complexity}

It takes approximately seventy times (run on a SUN-SLC workstation) the real time system to provide the perceptual-domain representation for a speech signal. However, most of the signal processing tasks (except the coupling stages) may be performed in parallel to make the operation real-time. Advances in VLSI and signal processing technology have resulted in the fabrication of an application-specific integrated circuit (ASIC) for cochlea [119]. An application of such an ASIC would make the processing very fast compared to the software simulation.

\subsubsection{Administration of Formal Subjective Test}

An objective measure is considered to be useful if its result comports with the result of a formal subjective test (generally, the MOS). A regression analysis is usually performed to determine an analytic relationship between the objective measures and the MOS scores. Since different coded signals with accompanying MOS scores werc not available in our academic environment, we had to rely on the results of informal test with twelve listeners. As a consequence of this, we have not carried out any regression analysis because finding a relationship between our objective measure and any such informal listening test result would only be misleading. With a limited timeduration for doctoral work, we had to make a choice between the two-(i) confining to the CDI measure approach and pursuing a more rigorous testing, or (ii) along with the CDI measure, addressing the issues of temporal ordering in the firing pattern and robustness of the measure against coder delays. The second option appealed to us. Although our experimental results show enough promise, correlation with a formal subjective test result is needed to validate our approach.

In a speech coder standardization process, the perceptual qualities of several coders are evlauated by subjective testing. Often, the coders are assessed under different test conditions. For example, the Telecommunications Industries Association (TIA) is currently setting up a $6.5 \mathrm{kbps}$ speech coder standard (half-rate North American standard) for mobile communication purposes [120]. From a large pool of 
candidate coders, they have selected nine of them. All these coders are being tested subjectively under fourteen different conditions (e.g., channel conditions, background noise, tandemming). Such a testing procedure involves a great deal of money and also consumes a large amount of time. We believe that our measures could, at least, be used in bringing down the number of candidate coders to a few for final subjective assessment. This would substantially reduce the amount of time and money involved for testing.

\subsubsection{Derivation of Firing Pattern}

For continuous speech, its perception depends not only on the acoustic cues, but also on the semantic cues (the meaning of preceding and following words and the subject matter), the syntactic cues (grammatical rules) and the circumstantial cues (speaker identity, listening environment etc.). It is quite likely that the processing of speech does not occur in a hierarchical way from one level to the next and that there are extensive links between levels [43]. However, the speech coders typically do not produce distortions that are specifically related to the semantic, syntactic or circumstantial cues. Therefore, it is reasonable to hypothesize that the proposed measures are, by and large, sufficient from this perspective.

The CDI measure compares the probability-of-firing information whereas the CHM measure compares implicitly the neural firing patterns for the original and the coded signals. With further progress in psychoacoustic research, it may be possible to derive the actual neural firing patterns from the cochlear model outputs by a suitable trigger mechanism [121] and compare them explicitly for the original and coded signals. Since all the information related to the speech perception are conveyed to the brain only as a sequence of neural firings through neural fibers, in future, an explicit comparison of these patterns may become an effective way for devising a distortion measure.

\subsubsection{Application of Measures in Speech Coding}

The present day state-of-the-art low bit-rate speech coders generally use, in the closedloop analysis, a mean square error criterion with some form of perceptual weighting filter. For an use of the introduced measures in a speech coding process, the cochlear 
model transformation has to be expressed in a more analytically tractable form. Also, because of the temporal masking effects, speech coding with such measures would imply additional coding delays. Motivated by the in-synchrony characteristics of the timing information in the auditory nerve firing patterns, in [122], the in-synchronybands spectrum has been used in an analysis/synthesis system. In [123], wavelet functions have been used to incorporate the multiresolution signal nature at the cochlea and represent the speech signal onto a joint time-frequency domain. Recent efforts (e.g., $[124,125])$ have been made to propose empirical but perceptually advantageous time-frequency frameworks for speech processing. Further research is necessary to express cochlear functions, preserving all the major perceptual events, in a compact mathematical form and apply it in the speech coding process.

\subsection{Epilog}

In this dissertation, our primary contributions are-(i) reviewing the existing subjective and objective distortion measures, (ii) studying the auditory system and various cochlear models, (iii) applying Lyon's cochlear model for auditory representation of speech, (iv) devising a cochlear discrimination information measure and evaluating speech coder performance with it, (v) pursuing a rate-distortion analysis with this measure for speech coding, (vi) formulating a cochlear hidden Markovian measure and assessing speech coder quality with it, (vii) suggesting an algorithm for pitch frequency estimation from the cochlear model outputs, (viii) comparing different perceptual weighting strategies adopted in low bit-rate speech coders and (ix) providing a future research direction in the context of our work.

Determining a 'good' distortion measure for speech coding is an extremely difficult problem due to its very basic nature. At the same time, finding such a measure would surely have a significant impact on the speech coding and coder evaluation procedures. Our objective has never been to give a 'final' answer for this complex problem, rather we have tried to take an incremental step towards the solution. With the progress of time, we expect an improvement of the cochlear model structure, a determination of an analytically tractable expression for it and also a reduction in the computational complexity. However, the basic framework of comparing the neural firing information for original and coded signal could still be maintained. 
Since the work of von Békésy, the auditory system has been studied from different perspectives. Some of these research findings are well-accepted in the literature. On the other side, since the pioneering work of Shannon, the field of information theory has grown substantially. Through the proposed work of distortion measures for coded speech, we have made an endeavor to use a physiological model for auditory processing and apply information-processing techniques from information theory. 


\section{Appendix A}

It is known [70,71] that $H_{\alpha}(P)$ is strictly concave with respect to $P$ for $0<\alpha \leq 1$, but its convexity or concavity depends on $J$ for $\alpha>1$. In this apperidix, we show that for $J=2$ (i.e., with $P=\left\{p_{1}, p_{2}\right\}$ ), (a) $H_{\alpha}(P)$ is strictly concave with respect to $P$ for $0<\alpha \leq 2$ and (b) for every $\alpha>2, H_{\alpha}(P)$ is neither convex nor concave with respect to $P$.

It is shown in [71] that the $H_{\alpha}(P)$ is concave for $0<\alpha \leq 1$. So, to prove (a), we have to show that for $J=2$, the concavity is also satisfied for $1<\alpha \leq 2$. We demonstrate this by showing that the second derivative of $H_{\alpha}(p,(1-p))$ with respect to $P$ is negative in the range $1<\alpha \leq 2$.

$$
\begin{aligned}
H_{\alpha}(P) & =\frac{1}{(1-\alpha)} \log \left(p_{1}^{\alpha}+p_{2}^{\alpha}\right), \quad \text { where } p_{2}=1-p_{1}, \quad p_{1}, p_{2} \geq 0 \\
\frac{d^{2} H_{\alpha}(P)}{d p_{1}^{2}} & =\frac{\alpha}{(1-\alpha)} \cdot \frac{(\alpha-1)\left(p_{1}^{\alpha}+p_{2}^{\alpha}\right)\left(p_{1}^{\alpha-2}+p_{2}^{\alpha-2}\right)-\alpha\left(p_{1}^{\alpha-1}-p_{2}^{\alpha-1}\right)^{2}}{\left(p_{1}^{\alpha}+p_{2}^{\alpha}\right)^{2}} \\
& =\frac{\alpha}{(1-\alpha)} \cdot \frac{\left(p_{1}^{\alpha-2} p_{2}^{\alpha-2}\right) \cdot\left\{\alpha-\left(p_{1}^{\alpha}+p_{2}^{\alpha}\right)\left(p_{1}^{2-\alpha}+p_{2}^{2-\alpha}\right)\right\}}{\left(p_{1}^{\alpha}+p_{2}^{\alpha}\right)^{-2}}
\end{aligned}
$$

It is noted that for $\alpha>1$,

$$
\left(p_{1}^{\alpha}+p_{2}^{\alpha}\right)<\left(p_{1}+p_{2}\right)^{\alpha}=1 .
$$

Furthermore, $p_{1}=p_{2}=1 / 2$ maximizes the expression $\left(p_{1}^{2-\alpha}+p_{2}^{2-\alpha}\right)$ for $1<\alpha \leq 2$. We note that $\alpha>\left(\frac{1}{2}\right)^{1-\alpha}$ for $1<\alpha \leq 2$. Additionally, we observe that the denominator factor $(1-\alpha)$ of (A.2) is negative for $\alpha>1$. Thus, $H_{\alpha}(P)$ is proved to be concave in the range $1<\alpha \leq 2$.

Now, we investigate the concavity for $J=2$ and $\alpha>2$. With sufficiently small $\delta>0$ and $p_{1}=\delta$ or $p_{2}=\delta$, we obtain

$$
\frac{d^{2} H_{\alpha}(P)}{d p_{1}^{2}}>0
$$

On the other hand, with $p_{1}=p_{2}=1 / 2$, we have

$$
\frac{d^{2} H_{\alpha}(P)}{d p_{1}^{2}}=-4 \alpha<0
$$

From (A.4) and (A.5), we observe that for $J=2$ and $\alpha>2, H_{\alpha}(P)$ is neither convex nor concave. 


\section{Appendix B}

In this appendix, we show that (a) the directed divergence measure based on the Rényi-Shannon entropy is non-negative and (b) it is additive for random measurements that are independent under both probability distributions. For showing the part (a), the inequality $\log x \geq 1-(1 / x)$ is used.

(a) Non-negativity of the measure:

$$
\begin{gathered}
D_{1}\left(P_{k l} ; Q_{k l}\right)=\sum_{j=1}^{2} p_{j k l} \log \left(\frac{p_{j k l}}{q_{j k l}}\right) \geq \sum_{j=1}^{2} p_{j k l}\left(1-\frac{q_{j k l}}{p_{j k l}}\right)=\sum_{j=1}^{2} p_{j k l}-\sum_{j=1}^{2} q_{j k l}=0 . \\
D_{\alpha}\left(P_{k l} ; Q_{k l}\right)=\frac{1}{\alpha-1} \log \left(\sum_{j=1}^{2} \frac{p_{j k l}^{\alpha}}{q_{j k l}^{\alpha-1}}\right) \geq \frac{1}{\alpha-1}\left[1-\frac{1}{\sum_{j=1}^{2} \frac{p_{j k l}^{\alpha} \frac{1}{q_{j k l}-1}}{\alpha-1}}\right], \quad \alpha \neq 1 .
\end{gathered}
$$

To show that $D_{\alpha}\left(P_{k l} ; Q_{k l}\right) \geq 0$, we need to show that

$$
\begin{aligned}
Y_{\alpha}\left(P_{k l} ; Q_{k l}\right) \equiv \sum_{j=1}^{2} \frac{p_{j k l}^{\alpha}}{q_{j k l}^{\alpha-1}} & \leq 1 \text { for } 0 \leq \alpha<1 \\
& \geq 1 \text { for } \alpha>1 .
\end{aligned}
$$

We note that $p_{1 k l}=q_{1 k l}$ and $p_{2 k l}=q_{2 k l}$ maximizes $Y_{\alpha}\left(P_{k l} ; Q_{k l}\right)$ for $0 \leq \alpha<1$ and minimizes it for $\alpha>1$. Thus, the $Y_{\alpha}\left(P_{k l} ; Q_{k l}\right)$ conditions of (B.3) are met and hence the non-negativity of the divergence measure (the Rényi-Shannon type) is also satisfied. The measure becomes equal to zero if and only if the distributions $P_{k l}$ and $Q_{k l}$ become the same.

(b) Addivitivity of the measure: With $w \in \mathcal{L} \times \mathcal{K}$ and $m=n N$, we obtain

$$
\begin{aligned}
D_{1}(P ; Q) & =\sum_{j_{1}=1}^{2} \sum_{j_{2}=1}^{2} \cdots \sum_{j_{m}=1}^{2}\left(\prod_{w=1}^{m} p_{j_{w}}\right)\left[\sum_{w=1}^{m} \log \frac{p_{j_{w}}}{q_{j_{w}}}\right] \\
& =\left\{\sum_{j_{1}=1}^{2} p_{j_{1}} \log \left(\frac{p_{j_{1}}}{q_{j_{1}}}\right) \sum_{j_{2}=1}^{2} p_{j_{2}} \cdots \sum_{j_{m}=1}^{2} p_{j_{m}}\right. \\
& +\sum_{j_{1}=1}^{2} p_{j_{1}} \sum_{j_{2}=1}^{2} p_{j_{2}} \log \left(\frac{p_{j_{2}}}{q_{j_{2}}}\right) \cdots \sum_{j_{m}=1}^{2} p_{j_{m}} \\
& \left.+\cdots+\sum_{j_{1}=1}^{2} p_{j_{1}} \sum_{j_{2}=1}^{2} p_{j_{2}} \cdots \sum_{j_{m}=1}^{2} p_{j_{m}} \log \left(\frac{p_{j_{m}}}{q_{j_{m}}}\right)\right\}
\end{aligned}
$$




$$
\begin{aligned}
& =\sum_{w=1}^{m}\left\{\sum_{j_{w}=1}^{2} p_{j_{w}} \log \left(\frac{p_{j_{w}}}{q_{j_{w}}}\right)\right\} \\
& =\sum_{l \in \mathcal{L}} \sum_{k \in \mathcal{K}} D_{1}\left(P_{k l} ; Q_{k l}\right)
\end{aligned}
$$

Similarly, for $\alpha \neq 1, \alpha \geq 0$, we get

$$
\begin{aligned}
D_{\alpha}(P ; Q) & =\frac{1}{(\alpha-1)} \log \left\{\sum_{j_{1}=1}^{2} \cdots \sum_{j_{m}=1}^{2}\left(\frac{\prod_{w=1}^{m} p_{j_{w}}^{\alpha}}{\prod_{w=1}^{m} q_{j_{w}}^{\alpha-1}}\right)\right\} \\
& =\frac{1}{(\alpha-1)} \log \left\{\left(\sum_{j_{1}=1}^{2} \frac{p_{j_{1}}^{\alpha}}{q_{j_{1}}^{\alpha-1}}\right)\left(\sum_{j_{2}=1}^{2} \frac{p_{j_{2}}^{\alpha}}{q_{j_{2}}^{\alpha-1}}\right) \cdots\left(\sum_{j_{m}=1}^{2} \frac{p_{j_{m}}^{\alpha}}{q_{j_{m}}^{\alpha-1}}\right)\right\} \\
& =\sum_{w=1}^{m}\left\{\frac{1}{(\alpha-1)} \log \left(\sum_{j=1}^{2} \frac{p_{j_{w}}^{\alpha}}{q_{j_{w}}^{\alpha-1}}\right)\right\} \\
& =\sum_{l \in \mathcal{C}} \sum_{k \in \mathcal{K}} D_{\alpha}\left(P_{k l} ; Q_{k l}\right)
\end{aligned}
$$




\section{Appendix C}

The reference audio files were obtained by digitally filtering the speech and sampling it at a rate of $8,000 \mathrm{~Hz}$. The digital filter (255 tap FIR) applied was designed to be unity between 0 and 3,200 Hz. For the purpose of speech coder evaluation, the following test sentences [male (M) and female (F) voices] were used.

1. Add the sum to the product of these three (M1, F1).

2. Cats and dogs each hate the other (M2, F2).

3. Oak is strong and also gives shade (M3, F3).

4. Open the crate but don't break the glass (M4, F.1).

5. The pipe began to rust while new (M5, F5).

6. Thieves who rob friends deserve jail (M6, F6). 


\section{Appendix D}

A beta density function is given as

$$
b(x)=\frac{\Gamma(d+f+2)}{\Gamma(d+1) \Gamma(f+1)} x^{d}(1-x)^{f} .
$$

In this appendix, we prove that this function satisfies the iug-concavity condition, i.e., the logarithm of the function is concave. Taking logarithm of (D.1), we get,

$$
\begin{aligned}
\phi(x)= & \log \Gamma(d+f+2)-\log \Gamma(d+1)-\log \Gamma(f+1) \\
& +d \log x+f \log (1-x)
\end{aligned}
$$

To show the log-concavity nature of (D.1), we need to show that $\phi(x)$ is concave w.r.t. $x$. Defining $\bar{\lambda} \equiv(1-\lambda)$, we write

$$
\begin{aligned}
& \phi\left(\lambda x^{\prime}+\bar{\lambda} x^{\prime \prime}\right)-\lambda \phi\left(x^{\prime}\right)-\bar{\lambda} \phi\left(x^{\prime \prime}\right) \\
= & d \lambda \log \left(\lambda x^{\prime}+\bar{\lambda} x^{\prime \prime}\right)+f \lambda \log \left(1-\lambda x^{\prime}-\bar{\lambda} x^{\prime \prime}\right)+d \bar{\lambda} \log \left(\lambda x^{\prime}+\bar{\lambda} x^{\prime \prime}\right) \\
& +f \bar{\lambda} \log \left(1-\lambda x^{\prime}-\bar{\lambda} x^{\prime \prime}\right)-d \lambda \log x^{\prime}-f \lambda \log \left(1-x^{\prime}\right) \\
& -d \bar{\lambda} \log x^{\prime \prime}-f \bar{\lambda} \log \left(1-x^{\prime \prime}\right) \\
= & d \lambda \log \left(\frac{\lambda x^{\prime}+\bar{\lambda} x^{\prime \prime}}{x^{\prime}}\right)+f \lambda \log \left(\frac{1-\lambda x^{\prime}-\bar{\lambda} x^{\prime \prime}}{1-x^{\prime}}\right) \\
& +d \bar{\lambda} \log \left(\frac{\lambda x^{\prime}+\bar{\lambda} x^{\prime \prime}}{x^{\prime \prime}}\right)+f \bar{\lambda} \log \left(\frac{1-\lambda x^{\prime}-\bar{\lambda} x^{\prime \prime}}{1-x^{\prime \prime}}\right) \\
\geq & d \lambda\left(1-\frac{x^{\prime}}{\lambda x^{\prime}+\bar{\lambda} x^{\prime \prime}}\right)+f \lambda\left(1-\frac{1-x^{\prime}}{1-\lambda x^{\prime}-\bar{\lambda} x^{\prime \prime}}\right) \\
& +d \bar{\lambda}\left(1-\frac{x^{\prime \prime}}{\lambda x^{\prime}+\bar{\lambda} x^{\prime \prime}}\right)+f \bar{\lambda}\left(1-\frac{1-x^{\prime \prime}}{1-\lambda x^{\prime}-\bar{\lambda} x^{\prime \prime}}\right) \\
= & d-d\left(\frac{\lambda x^{\prime}+\bar{\lambda} x^{\prime \prime}}{\lambda x^{\prime}+\bar{\lambda} x^{\prime \prime}}\right)+f-f\left(\frac{\lambda\left(1-x^{\prime}\right)+\bar{\lambda}\left(1-x^{\prime \prime}\right)}{1-\lambda x^{\prime}+\bar{\lambda} x^{\prime \prime}}\right) \\
= & 0 .
\end{aligned}
$$

Since it has been shown that $\phi\left(\lambda x^{\prime}+\bar{\lambda} x^{\prime \prime}\right) \geq \lambda \phi\left(x^{\prime}\right)-\bar{\lambda} \phi\left(x^{\prime \prime}\right)$, the beta pdf of (D.1) is proven to be log-concave. 


\section{Appendix E}

In our work, an auxiliary function $F\left(\lambda, \lambda^{\prime}\right)$ is considered as the basis for the maximum likelihood optimization procedure. The Baum-Welch (re)estimation procedure is used for determining different model parameters. Separability of the individual auxiliary functions has made this procedure elegant and reduced the complexity. Here, we write the expressions for individual auxiliary functions. We can rewrite (5.25) as

$$
\begin{aligned}
F\left(\lambda, \lambda^{\prime}\right)= & F_{\pi}\left(\lambda, \pi^{\prime}\right)+\sum_{i \in \mathcal{N}} F_{a_{i}}\left(\lambda,\left\{a_{i j}^{\prime}\right\}_{j \in \mathcal{N}}\right) \\
& +\sum_{i \in \mathcal{N}} \sum_{m \in \mathcal{M}_{L}} F_{b}\left(\lambda, b_{i m}^{\prime}\right)+\sum_{i \in \mathcal{N}} F_{c_{i}}\left(\lambda,\left\{c_{i m}^{\prime}\right\}_{m \in \mathcal{M}_{L}}\right),
\end{aligned}
$$

where

$$
\begin{aligned}
& F_{\pi}\left(\boldsymbol{\lambda}, \pi^{\prime}\right)=\sum_{Q \in \mathcal{N}^{T}} \sum_{M \in \mathcal{M}_{L}^{T}} P\left(O, Q, N_{i} \mid \lambda\right) \log \pi_{q_{1}}^{\prime} \\
& =\sum_{i \in \mathcal{N}} \sum_{M \in \mathcal{M}_{L}^{T}} P\left(\boldsymbol{O}, q_{1}=S_{i}, M \mid \lambda\right) \log \pi_{i}^{\prime}, \\
& F_{a_{i}}\left(\boldsymbol{\lambda},\left\{a_{i j}^{\prime}\right\}_{j \in \mathcal{N}}\right)=\sum_{Q \in \mathcal{N}^{T}} \sum_{M \in \mathcal{M}_{L}^{T}} P(O, Q, M \mid \lambda) \sum_{t \in \mathcal{T}^{+}} \log a_{q_{t} q_{t+1}}^{\prime} \delta\left(q_{t}-S_{i}\right) \\
& =\sum_{j \in \mathcal{N}} \sum_{t \in \mathcal{T}^{+}} \sum_{M \in \mathcal{M}_{L}^{T}} P\left(O, q_{t}=S_{i}, q_{t+1}=S_{j}, M \mid \lambda\right) \log a_{i j}^{\prime}, \\
& F_{b}\left(\boldsymbol{\lambda}, b_{i m}^{\prime}\right)=\sum_{Q \in \mathcal{N}^{T}} \sum_{M \in \mathcal{M}_{L}^{T}} P(O, Q, M \mid \lambda) \cdot \sum_{t \in \mathcal{T}^{+}} \log b_{q_{t} m_{\mathrm{t}}}^{\prime}\left(O_{t}\right) \delta\left(q_{t}-S_{i}\right) \delta\left(m_{t}-m\right) \\
& =\sum_{t \in \mathcal{T}^{+}} P\left(O, q_{t}=S_{i}, m_{t}=m \mid \boldsymbol{\lambda}\right) \log b_{i m}^{\prime}\left(O_{t}\right)
\end{aligned}
$$

and

$$
\begin{aligned}
F_{c_{i}}\left(\boldsymbol{\lambda},\left\{c_{i m}^{\prime}\right\}_{m \in \mathcal{M}_{L}}\right) & =\sum_{Q \in \mathcal{N}^{T}} \sum_{M \in \mathcal{M}_{L}^{T}} P(O, Q, M \mid \lambda) \sum_{t \in \mathcal{T}^{+}} \log c_{q_{t} m_{t}}^{\prime} \delta\left(q_{t}-S_{i}\right) \\
& =\sum_{m \in \mathcal{M}_{L}} \sum_{t \in \mathcal{T}^{+}} P\left(O, q_{t}=S_{i}, m_{t}=m \mid \boldsymbol{\lambda}\right) \log c_{i m}^{\prime},
\end{aligned}
$$

where $\delta$ in the above expressions is the Kronecker delta function. 


\section{References}

[1] R. E. Blahut, Principles and Practice of Information Theory. Addison-Wesley, 1987.

[2] D. O'Shaughnessy, Speech Communication. Academic Press, 1987.

[3] N. S. Jayant and P. Noll, Digital Coding of Waveforms: Principles and Applications to Speech and Video. Prentice Hall, 1984.

[4] L. Rabiner and R. Schafer, Digital Processing of Speech Signals. Prentice Hall, 1979.

[5] J. P. Campbell Jr., T. E. Tremain, and V. C. Welch, "The federal standard 1016 4800 bps CELP voice coder," Digital Signal Processing, vol. 1, pp. 145-155, July 1991.

[6] W. B. Kleijn, Analysis-by-Synthesis Speech Coding Based on Relaxed WaveformMatching Constraints. PhD thesis, Delft University of Technology, Dec. 1991.

[7] J.-P. Adoul, P. Mabilleau, M. Delprat, and S. Morissette, "Fast CELP coding based on algebraic codes," in Proc. IEEE Int. Conf. Acoust., Specch and Signal Process., pp. 49.4.1-49.4.4, 1987.

[8] A. Gersho and R. M. Gray, Vecior Quantization and Signal Compression. Kluwer Academic Pub., 1992.

[9] K. Abboud, "Wideband CELP speech coding," Master's thesis, McGill University, Feb. 1993.

[10] H. J. Coetzee and T. P. Barnwell III, "An LSP based speech quality measure," in Proc. IEEE Int. Conf. Acoust., Speech and Signal Process., pp. 596-599, 1989. 
[11] S. R. Quackenbush, T. P. Barnwell III, and M. A. Clements, Objective Measures of Speech Quality. Prentice Hall, 1988.

[12] T. P. Barnwell III, "Correlation analysis of subjective and objective measures for speech quality, in Proc. IEEE Int. Conf. Acoust., Speech and Signal Process., pp. $706-709,1980$.

[13] R. F. Kubichek, "Standards and technology issues in objective voice quality assessment," Dig. Signal Process., vol. 1, pp. 38-44, Jan. 1991.

[14] M. H. L. Hecker and C. E. Williams, "Choice of reference conditions for speech preference tests," J. Acoust. Soc. Am., vol. 40, pp. 946-952, Nov. 1966.

[15] H. Fletcher and J. C. Steinberg, "Articulation testing methods," Bell Syst. Tech. J., pp. 806-854, 1929.

[16] G. Fairbanks, "Test of phonemic differertiation: The rhyme test," J. Acoust. Soc. Am., pp. 596-600, July 1958.

[17] W. A. Munson and J. E. Karlin, "Isopreference method for evaluating speechtransmission circuits," J. Acoust. Soc. Am., vol. 31, pp. 762-774, June 1962.

[18] "IEEE recommended practice for speech quality measurements," IEEE Trans. Aud. and Electroacoust., pp. 227-246, Sept. 1969.

[19] W. D. Voiers, "Diagnostic acceptability measure for speech communication systems," in Proc. IEEE Int. Conf. Acoust., Speech and Signal Process., pp. 204$207,1977$.

[20] S. R. Quackenbush, "Objective measures of speech quality," Tech. Rep. DSPL85-4. Seorgia Institute of Technology, 1985.

[21] M. R. Schroeder, B. S. Atal, and J. L. Hall, "Optimizing digital speech coders by exploiting masking properties of the human ear," J. Acoust. Soc. Am., vol. 66, pp. 1647-1652, Dec. 1979.

[22] P. Mermelstein, "Evaluation of a segmental SNR measure as an indicator of the quality of ADPCM coded speech," J. Acoust. Soc. Am., vol. 66, pp. 1664-1667, Dec. 1979. 
[23] B. J. McDermott, C. Scagliola, and D. J. Goodman, "Perceptual and objective evaluation of speech processed by adaptive differential PCM," Bell Syst. Tech. J., pp. 1597-1619, May 1978.

[24] R. E. Crochiere, J. E. Tribolet, and L. R. Rabiner, "An interpretation of the $\log$ likelihood ratio as a measure of waveform coder performance," IEEE Trans. Acoust., Speech and Signal Process., vol. ASSP-28, pp. 318-323, June 1980.

[25] R. M. Gray, A. Buzo, A. H. Gray Jr., and Y. Matsuyama, "Distortion measures for speech processing," IEEE Trans. Acoust., Speech and Signal Process., vol. ASSP-28, pp. 367-376, Aug. 1980.

[26] U. Halka and U. Heute, "A new approach to objective quality-measures based on attribute-matching," Speech Commun., vol. 11, pp. 15-30, Mar. 1992.

[27] A. H. Gray and J. D. Markel, "Distance measures for speech processing," IEEE Trans. Acoust., Speech and Signal Process., vol. ASSP-24, pp. 380-391, Oct. 1976.

[28] N. Kitawaki, H. Nagabuchi, and K. Itoh, "Objective quality evaluation for low-bit-rate speech coding systems," IEEE J. Select. Areas Commun., vol. 6, pp. 242-248, Feb. 1988.

[29] K. K. Paliwal, "On the performance of the quefrency-weighted cepstral coefficients in vowel recognition," Speech Commun., vol. 1, pp. 151-154, May 1982.

[30] Y. Tohkura, "A weighted cepstral distance measure for speech recognition," IEEE Trans. Acoust., Speech and Signal Process., vol. ASSP-35, pp. 1414-1422, Oct. 1987.

[31] Y.-T. Lee, "Information-theoretic distortion measures for specch recognition," IEEE Trans. Signal Process., vol. 39, pp. 330-335, Feb. 1991.

[32] F. Itakura and S. Saito, "Analysis synthesis telephony based on the maximum likelihood method," in Proc. 6th Int. Cong. Acoust., Japan, pp. C 17-C 20, 1968.

[33] P. L. Chu and D. G. Messerschmitt, "A frequency weighted Itakura-Saito spectral distance measure," IEEE Trans. Acoust., Speech and Signal Process., vol. ASSP-30, pp. 545-560, Aug. 1982. 
[34] B. A. Carlson and M. A. Clements, "A computationally compact divergence measure for speech processing," IEEE Trans. Pattern Anal. and Machine Intell., vol. 13, pp. 1255-1260, Dec. 1991.

[35] Bell Northern Research, "Evaluation of nonlinear distortion via the coherence function," Contribution to CCITT, COM-XII-no. 60-E, Apr. 1982.

[36] J. Lalou, "The information index: An objective measure of speech transmission performance," Ann. Telecommun., vol. 45, pp. 47-65, Jan. 1990.

[37] S. Wang, A. Sekey, and A. Gersho, "An objective measure for predicting subjective quality of speech coders," IEEE J. Select. Areas Commun., vol. 10, pp. 819-829, June 1992.

[38] R. Bladon, "Modeling the judgement of vowel quality differences," J. Acoust. Soc. Am., vol. 69, pp. 1414-1422, May 1981.

[39] D. Robinson and R. Dadson, "A redetermination of the equal-loudness relations for pure tones," Brit. J. Appl. Physics, vol. 7, pp. 166-181, 1956.

[40] B. Paillard, J. Soumagne, P. Mabilleau, and S. Morissette, "PERCEVAL: Perceptual evaluation of the quality of audio signals," J. Audio Eng. Soc., vol. 40, pp. 21-31, Jan.-Feb. 1992.

[41] J. Flanagan, Speech Analysis, Synthesis and Perception. Springer-Verlag, 1972.

[42] D. Green, An Introduction to Hearing. Erlbaum, 1976.

[43] B. C. J. Moore, Introduction to the Psychology of Hearing. Academic Press, 1989.

[44] R. F. Lyon, "A computational model of filtering, detection, and compression in the cochlea," in Proc. IEEE Int. Conf. Acoust., Speech and Signal Process., pp. 1282-1285, 1982.

[45] G. von Békésy, Experiments in Hearing. McGraw-Hill, 1960.

[46] J. B. Allen, "Cochlear modeling," IEEE ASSP Mag., pp. 3-29, Jan. 1985.

[47] J. J. Zwislocki, "Five decades of research on cochlear mechanics," J. Acoust. Soc. Am., vol. 67, pp. 1679-1685, 1980. 
[48] D. T. Kemp, "Towards a model for the origin of cochlear echoes," Hearing Res., vol. 2, pp. 533-548, 1980.

[49] M. J. Penner, "Forward masking with equal-energy maskers," J. Acoust. Soc. Am., vol. 66, pp. 1719-1724, Dr 1979.

[50] E. D. Young and P. E. Barta, "Rate responses of auditory nerve fibers to tones in noise near masked threshold," J. Acoust. Soc. Am., vol. 79, pp. 426-442, Feb. 1986.

[51] A. B. Carlson, Communication Systems. McGraw Hill, 1986.

[52] H. V. Helmholtz, On the Sensations of Tone. Dover Pub., 1954.

[53] C. D. Geisler, "Representation of speech sounds in the auditory nerve," J. of Phonetics, vol. 16, pp. 19-35, Jan. 1988.

[54] S. Greenberg, "The ear as a speech analyzer," J. of Phonetics, vol. 16, pp. 139149, Jan. 1988.

[55] M. B. Sachs, C. C. Blackburn, and E. D. Young, "Rate-place and temporalplace representations of vowels in the auditory nerve and anteroventral cochlear nucleus," J. of Phonetics, vol. 16, pp. 37-53, Jan. 1988.

[56] S. Seneff, "A joint synchrony/mean-rate model of auditory speech processing," J. of Phonetics, vol. 16, pp. 55-76, Jan. 1988.

[57] S. A. Shamma, "Speech processing in the auditory system II: Lateral inhibition and the central processing of speech evoked activity in the auditory nerve," $J$. Acoust. Soc. Am., vol. 78, pp. 1622-1632, Nov. 1985.

[58] O. Ghitza, "Temporal non-place information in the auditory-nerve firing patterns as a front-end for speech recognition in a noisy environment," J. of Phonetics, vol. 16, pp. 109-123, Jan. 1988.

[59] L. Deng, C. D. Geisler, and S. Greenberg, "A composite model of the auditory periphery for the processing of speech," J. of Phonetics, vol. 16, pp. 93-108, Jan. 1988.

[60] G. Zweig, R. Lipes, and J. R. Pierce, "The cochlear compromise," J. Acoust. Soc. Am., vol. 59, pp. 975-982, 1976. 
[61] M. Slaney, "Lyon's cochlear model," Tech. Rep. 13, Apple Computer Inc., 1988.

[62] J. O. Pickles, An Introduction to the Physiology of Hearing. Academic Press, 1982.

[63] L. Deng, "Processing of acoustic signals in a cochlear model incorporating laterally coupled suppressive elements," Neural Networks, vol. 5, pp. 19-34, 1992.

[64] T. Hall, "Cochlear models: Evidence in support of mechanical nonlinearity and second filters (a review)," Hearing Res., vol. 2, pp. 455-464, 1980.

[65] M. R. Schroeder and J. L. Hall, "Model for mechanical to neural transduction in the auditory receptor," J. Acoust. Soc. Am., vol. 55, pp. 1055-1060, 1974.

[66] R. F. Lyon and L. Dyer, "Experiments with a computational model of the cochlea," in Proc. IEEE Int. Conf. Acoust., Speech and Signal Process., pp. 37.6.1-37.6.4, 1986 .

[67] S. Kullback, Information Theory and Statistics. John Wiley \& Sons, 1959.

[68] A. De and P. Kabal, "Cochlear discrimination: An auditory informationtheoretic distortion measure for speech coders," in Proc. 16 th Biennial Symp. on Commun., Kingston, Canada, pp. 419-423, May 1992.

[69] A. De and P. Kabal, "Auditory distortion measure for coded speechdiscrimination information approach," Speech Commun. (being revised for publication), 1993.

[70] J. Aczél and Z. Daróczy, On Measures of Information and Their Characterizations. Academic Press, 1975.

[71] A. Rényi, Probability Theory. North-Holland, 1970.

[72] J. Aczél, "Some recent results on characterizations of measures of information related to coding," IEEE Trans. Inform. Theory, vol. IT-24, pp. 592-595, Sept. 1978.

[73] J. Lin, "Divergence measures based on the Shannon entropy," IEEE Trans. Inform. Theory, vol. 37, pp. 145-151, Jan. 1991. 
[74] C. R. Rao and T. K. Nayak, "Cross entropy, dissimilarity measures, and characterizations of quadratic entropy," IEEE Trans. Inform. Theory, vol. IT-31, pp. 589-593, Sept. 1985.

[75] G. T. Toussaint, "Sharper lower bounds for discrimination information in terms of variation," IEEE Trans. Inform. Theory, vol. IT-21, pp. 99-100, Jan. 1975.

[76] R. E. Blahut, "Hypothesis testing and information theory," IEEE Trans. Inform. Theory, vol. IT-20, pp. 405-417, July 1974.

[77] T. B. Berger, Rate Distortion Theory. Prentice Hall, 1971.

[78] D. J. Sakrison, "The rate distortion function of a Gaussian process with a weighted square error criterion," IEEE Trans. Inform. Theory, vol. IT-14, pp. 506-508, May 1968.

[79] A. Buzo, F. Kuhlmann, and C. Rivera, "Rate-disiortion bounds for quotientbased distortions with applications to Itakura-Saito distortion measures," IEEE Trans. Inform. Theory, vol. IT-32, pp. 141-147, Mar. 1986.

[80] J. T. Pinkston, "An application of rate-distortion theory to a converse to the coding theorem," IEEE Trans. Inform. Theory, vol. IT-15, pp. 66-71, Jan. 1969.

[81] R. M. Gray, "Rate distortion functions for finite-state finite-alphabet Markov sources," IEEE Trans. Inform. Theory, vol. IT-17, pp. 127-134, Mar. 1971.

[82] H. H. Tan and K. Yao, "Evaluation of rate-distortion functions for a class of independent identically distributed sources under an absolute-magnitude criterion," IEEE Trans. Inform. Theory, vol. IT-21, pp. 59-64, Jan. 1975.

[83] R. E. Blahut, "Computation of channel capacity and rate-distortion functions," IEEE Trans. Inform. Theory, vol. IT-18, pp. 460-473, July 1972.

[84] P. Noll, "Adaptive quantizing in speech coding systems," in Zurich Seminar Dig. Commun., Zurich, Switzerland, Mar. 1974.

[85] D. H. Richards, "Statistical properties of speech signals," Proc. IEEE, vol. 52, pp. 941-949, 1964.

[86] H. Abut and N. Erdöl, "Bounds on $R_{1}(D)$ functions for speech probability models," IEEE Trans. Inform. Theory, vol. IT-25, pp. 225-228, Mar. 1979. 
[87] A. De and P. Kabal, "Rate distortion function for speech coding based on perceptual distortion measure, ${ }^{n}$ in Proc. of IEEE Globecom'92, pp. 452-456, Dec. 1992.

[88] B. S. Atal, V. Cuperman, and A. Gersho, Advances in Speech Coding. Kluwer Academic Pub., 1991.

[89] G. Roy and P. Kabal, "Wideband CELP speech coding at $16 \mathrm{kbits} / \mathrm{sec}$, in Proc. IEEE Int. Conf. Acoust., Speech and Signal Process., pp. 17-20, 1991.

[90] A. De and P. Kabal, "Hidden Markov model-based auditory distortion measure for speech coder evaluation," in Abstracts of Canadian Inst. Telecommun. Res. Conf., Montréal, Canada (to appear), Aug. 1993.

[91] A. De and P. Kabal, "Auditory distortion measure for coded speech-hidden Markovian approach," Speech Commun. (being prepared for submission), 1993.

[92] L. E. Baum and T. Petrie, "Statistical inference for probabilistic functions of finite state Markov chains," Ann. Math. Stat., vol. 37, pp. 1554-1563, 1966.

[93] L. E. Baum and J. A. Egon, "An inequality with applications to statistical estimation for probabilistic functions of a Markov process and to a model for ecology," Bull. Amer. Meteorol. Soc., vol. 73, pp. 360-363, 1967.

[94] R. I. Cave and L. P. Neuwirth, "Hidden Markov models for english," Hidden Markov models for Speech (J. Ferguson [ed.]), vol. IDA-CRD, pp. 16-56, 1980.

[95] R. W. Chang and J. C. Hancock, "On receiver structures for channels having memory," IEEE Trans. Inform. Theory, vol. IT-12, pp. 463-468, Oct. 1966.

[96] F. Jelinek, "Continuous speech recognition by statistical methods," Proc. IEEE, vol. 64, pp. 532-536, Apr. 1976.

[97] L. A. Liporace, "Maximum likelihood estimation for multivariate observations of Markov sources," IEEE Trans. Inform. Theory, vol. IT-28, pp. 729-734, Sept. 1984.

[98] B.-H. Juang, "Maximum-likelihood estimation for mixture multivariate stochatic observations of Markov chains," Bell Syst. Tech. J., pp. 1235-1249, July-Aug. 1985. 
[99] L. E. Baum, "An inequality and associated maximization technique in statistical estimation for probabilistic functions of Markov processes," Inequalities, vol. 3, pp. 1-8, 1972.

[100] B.-H. Juang, "On the hidden Markov model and dynamic time warping for speech recognition," Bell Syst. Tech. J., pp. 1213-1243, Sept. 1984.

[101] S. E. Levinson, L. R. Rabiner, and M. M. Sondhi, "An introduction to the application of the theory of probabilistic functions of a Markov process to automatic speech recognition," Bell Syst. Tech. J., pp. 1035-1074, Apr. 1983.

[102] L. R. Rabiner, B.-H. Juang, S. E. Levinson, and M. M. Sondhi, "Some properties of continuous hidden Markov model representations," Bell Syst. Tech. J., pp. 1251-1269, July-Aug. 1985.

[103] W. Hess, Pitch Determination of Speech Signals: Algorithms and Devices. Springer-Verlag, 1983.

[104] M. Slaney and R. F. Lyon, "Visualizing sound with auditory correlograms," in submission for J. Acoust. Soc. Am., 1991.

[105] R. F. Lyon, "Computational models of neural auditory processing," in Proc. IEEE Int. Conf. Acoust., Speech and Signal Process., pp. 36.1.1-36.1.4, 1984.

[106] M. Weintraub, "A computational model for separating two simultaneous talkers," in Proc. IEEE Int. Conf. Acoust., Speech and Signal Process., 1986.

[107] S. Seneff, "Pitch and spectral estimation of speech based on auditory synchrony model," in Proc. IEEE Int. Conf. Acoust., Speech and Signal Process., pp. 36.2.1-36.2.4, 1984.

[108] P. Kabal and R. P. Ramachandran, "The computation of line spectral frequencies using Chebyshev polynomials," IEEE Trans. Acoust. Speech and Signal Process., vol. ASSP-34, pp. 1419-1426, 1986.

[109] Y. Linde, A. Buzo, and R. M. Gray, "An algorithm for vector quantizer design," IEEE Trans. Commun., vol. COM-28, pp. 84-95, Jan. 1980.

[110] J. L. Moncet and P. Kabal, "Codeword selection for CELP coders," Tech. Rep. 87-35, INRS-Telecommunications, 1987. 
[111] Q. Yasheng and P. Kabal, "Pseudo-three-tap pitch prediction filters," in Proc. IEEE Int. Conf. Acoust., Speech and Signal Process., pp. II.523-II.526, 1993.

[112] R. E. Crochiere and L. R. Rabiner, Multirate Digital Signal Processing. Prentice Hall, 1983.

[113] N. S. Jayant and V. Ramamoorthy, "Adaptive postfiltering of $16 \mathrm{~kb} / \mathrm{s}-$ adpcm speech," in Proc. IEEE Int. Conf. Acoust., Speech and Signal Process., pp. 16.4.1-16.4.4, 1986.

[114] C. Laflamme, J.-P. Adoul, R. Salami, S. Morissette, and P. Mabilleau, "16 kbps wideband speech coding technique based on algebraic CELP," in Proc. IEEE Int. Conf. Acoust., Speech and Signal Process., pp. 13-16, 1991.

[115] E. Ordentlich and Y. Shoham, "Low-delay code-excited linear-predictive coding of wideband speech at $32 \mathrm{kbps}$," in Proc. IEEE Int. Conf. Acoust., Speech and Signal Process., pp. 9-12, 1991.

[116] A. De, "Auditory distortion measures for coded speech quality evaluation," in Proc. Canadian Acoust. Assoc. Annual Symp., Toronto, Canada (to appear), Oct. 1993.

[117] J. M. Kates, "A time-domain digital cochlear model," IEEE Trans. Signal Process., vol. 39, pp. 2573-2592, Dec. 1991.

[118] T. Kohoner, Self-Organization and Associative Memory. Springer Verlag, 1988.

[119] R. F. Lyon and C. Mead, "An analog electronic cochlea," IEEE Trans. Acoust. Speech and Signal Process., vol. 36, pp. 1119-1134, July 1988.

[120] "Half-rate speech codec test plan V 6.0," Tech. Rep. TR 45.35, Telecommun. Industries Assoc., 1993.

[121] R. D. Patterson, "Auditory/connectionist techniques for speech," Tech. Rep. 2, ESPRIT Basic Research Action 3207, 1991.

[122] O. Ghitza, "Auditory nerve representation criteria for speech analysis/synthesis," IEEE Trans. Acoust., Speech and Signal Process., vol. ASSP-35, pp. 736-740, June 1987. 
[123] X. Yang, K. Wang, and S. A. Shamma, "Auditory representation of acoustic signals," IEEE Trans. Inform. Theory, vol. 38 (II), pp. \$24-839, Mar. 1992.

[124] Y. Shoham, "High quality speech coding at 2.4 to $4.0 \mathrm{kbps}$ based on timefrequency interpolation," in Proc. IEEE Int. Conf. Acoust., Speech and Signal Process., pp. II.167-II.170, 1993.

[125] D. Sen, D. H. Irving, and W. H. Holmes, "Use of an auditory inodel to improve speech codv: :s," in Proc. IEEE Int. Conf. Acoust., Speech and Signal Process., pp. II.411-II.414, 1993. 